\title{
Frobenius splitting of Schubert varieties of semi-infinite flag manifolds
}

\author{
Syu Kato \\ Department of Mathematics, Kyoto University, Oiwake Kita-Shirakawa, Sakyo, Kyoto 606-8502, Japan; \\ E-mail: syuchan@math.kyoto-u.ac.jp.
}

Received: 25 November 2019; Accepted: 24 April 2021

2020 Mathematics Subject Classification: Primary - 20G44; Secondary - 14N15, 14N35

\begin{abstract}
We exhibit basic algebro-geometric results on the formal model of semi-infinite flag varieties and its Schubert varieties over an algebraically closed field $\mathbb{K}$ of characteristic $\neq 2$ from scratch. We show that the formal model of a semi-infinite flag variety admits a unique nice (ind-)scheme structure, its projective coordinate ring has a $\mathbb{Z}$-model and it admits a Frobenius splitting compatible with the boundaries and opposite cells in positive characteristic. This establishes the normality of the Schubert varieties of the quasi-map space with a fixed degree (instead of their limits proved in [K, Math. Ann. 371 no.2 (2018)]) when char $\mathbb{K}=0$ or $\gg 0$, and the higher-cohomology vanishing of their nef line bundles in arbitrary characteristic $\neq 2$. Some particular cases of these results play crucial roles in our proof [47] of a conjecture by Lam, Li, Mihalcea and Shimozono [60] that describes an isomorphism between affine and quantum $K$-groups of a flag manifold.
\end{abstract}

\section{Introduction}

The semi-infinite flag varieties are variants of affine flag varieties that encode the modular representation theory of a semi-simple Lie algebra, representation theory of a quantum group at roots of unity and representation theory of an affine Lie algebra at the critical level. They originate from the ideas of Lusztig [64] and Drinfeld, put forward by Feigin and Frenkel [24] and subsequently polished by the work of Braverman, Finkelberg, and their collaborators [29, 23, 2, 7, 8, 9, 10, 11]. They (mainly) used the ind-model of semi-infinite flag varieties, and achieved spectacular success on the geometric Langlands correspondence [2, 12], the quantum $K$-groups of flag manifolds [9] and their (conjectural) relation to the finite $\mathcal{W}$-algebras [8].

In [51], we initiated the study of the formal model of a semi-infinite flag variety (over $\mathbb{C}$ ) that follows the classical description of flag varieties $[57,69,63,58]$ more closely than the works already cited. We refer to this formal model of a semi-infinite flag variety as a 'semi-infinite flag manifold', since we hope to justify that it is 'smooth' in a sense. However, the analysis in [51] has two defects: the relation with the ind-models of semi-infinite flag varieties is unclear, and the treatment there is rather ad hoc (it is just an ind-scheme whose set of $\mathbb{C}$-valued points have the desired property, and lacks a characterisation as a functor; compare [3, 22]). The first defect produces difficulty in discussing deep properties on the identification between the equivariant $K$-group of a semi-infinite flag manifold and the equivariant quantum $K$-group of a flag manifold [47], which is in turn inspired by the works of Givental and Lee $[32,33]$. The goal of this paper is to study semi-infinite flag manifolds in characteristic $\neq 2$ from scratch,

(C) The Author(s), 2021. Published by Cambridge University Press. This is an Open Access article, distributed under the terms of the Creative Commons Attribution licence (http://creativecommons.org/licenses/by/4.0/), which permits unrestricted re-use, distribution, and reproduction in any medium, provided the original work is properly cited. 
and resolve these defects. In particular, we verify that the scheme in [51] is universal one among all the ind-schemes with similar set-theoretic properties, and provide new proofs of the normality of Zastava spaces [9] and the semi-infinite flag manifolds [51].

It is possible to regard our work $([46,49,51,47,48])$ as part of catch-up of Peterson's original construction [74] of his isomorphism [61] between the quantum cohomology of a flag manifold and the cohomology of an affine Grassmannian in the $K$-theoretic setting. From this viewpoint, this paper provides some varieties considered in [74, Lecture 11] with their appropriate compactifications. Hence, though there are still some missing pieces to complete the original program along the lines in [74], this paper provides a step to fully examine Peterson's ideas.

To explain our results more precisely, we introduce more notation: Let $\mathfrak{g}$ denote a simple Lie algebra (given in terms of root data and the Chevalley generators) over an algebraically closed field $\mathbb{K}$ of characteristic $\neq 2$. Let $G$ denote the connected simply connected algebraic group over $\mathbb{K}$ such that $\mathfrak{g}=$ Lie $G$. Let $H \subset G$ be a Cartan subgroup and $N$ be an unipotent radical of $G$ that is normalised by $H$. We set $B:=H N$ and $\mathscr{B}:=G / B$ (the flag manifold of $G$ ). Let $\mathbf{I}^{+} \subset G(\mathbb{K}[[z]])$ denote the Iwahori subgroup that contains $B$, and let $\mathbf{I}^{-} \subset G\left(\mathbb{K}\left[z^{-1}\right]\right)$ be its opposite Iwahori subgroup. Let $\widetilde{\mathfrak{g}}$ denote the untwisted affine Kac-Moody algebra associated to $\mathfrak{g}$, and let $W$ and $W_{\text {af }}$ be the finite Weyl group and the affine Weyl group of $\mathfrak{g}$, respectively. The coroot lattice $Q^{\vee}$ of $\mathfrak{g}$ yields a natural subgroup $\left\{t_{\beta}\right\}_{\beta \in Q^{\vee}} \subset W_{\text {af }}$. Let $w_{0} \in W$ be the longest element.

Our first main result is as follows:

Theorem A ( $\doteq$ Theorem 4.18 and Proposition 4.26). There is an ind-scheme $\mathbf{Q}_{G}^{\text {rat }}$ with the following properties:

1. It is expressed as the union of infinite-type integral schemes flat over $\mathbb{Z}$.

2. If we set $\left(\mathbf{Q}_{G}^{\text {rat }}\right)_{\mathbb{K}}:=\mathbf{Q}_{G}^{\text {rat }} \otimes_{\mathbb{Z}} \mathbb{K}$, we have

$$
\left(\mathbf{Q}_{G}^{\text {rat }}\right)_{\mathbb{K}}(\mathbb{K}) \cong G(\mathbb{K}((z))) /(H(\mathbb{K}) N(\mathbb{K}((z)))),
$$

which intertwines the natural $G(\mathbb{K}((z))) \ltimes \mathbb{G}_{m}(\mathbb{K})$-actions on both sides, where $\mathbb{G}_{m}$ is the loop rotation.

3. The functor

$$
A f f_{\mathbb{K}}^{o p} \ni R \mapsto G(R((z))) /(H(R) N(R((z)))) \in \text { Sets }
$$

is coarsely ind-representable by $\left(\mathbf{Q}_{G}^{\mathrm{rat}}\right)_{\mathbb{K}}$ (see Section 4.3 for the convention).

One can equip $\left(\mathbf{Q}_{G}^{\text {rat }}\right)_{\mathbb{K}}(\mathbb{K})$ with an ind-scheme structure using the arc scheme of the basic affine space $\overline{G / N}$. Such an ind-scheme cannot coincide with ours (in general), by the appearance of the nontrivial nilradicals $[71,27,26]$. In fact, such an ind-scheme defines a radicial thickening of $\left(\mathbf{Q}_{G}^{\text {rat }}\right)_{\mathbb{K}}$.

The set of $H \times \mathbb{G}_{m}$-fixed points of $\left(\mathbf{Q}_{G}^{\text {rat }}\right)_{\mathbb{K}}$ is in bijection with $W_{\text {af }}$. Let $p_{w} \in\left(\mathbf{Q}_{G}^{\text {rat }}\right)_{\mathbb{K}}$ be the point corresponding to $w \in W_{\text {af }}$. We set $\mathbb{O}(w):=\mathbf{I}^{+} p_{w}$ and $\mathbb{O}^{-}(w):=\mathbf{I}^{-} p_{w}$ for each $w \in W_{\text {af }}$.

Theorem $\mathrm{A}$ has some applications to the theory of quasi-map spaces from $\mathbb{P}^{1}$ to $\mathscr{B}$ presented in [29, 23, 9, 10, 11] as follows:

Theorem B. In the foregoing settings, the following hold:

1. (Corollary 3.38 and Theorem 4.30) If char $\mathbb{K}>0$, then the scheme $\left(\mathbf{Q}_{G}^{\text {rat }}\right)_{\mathbb{K}}$ admits an $\mathbf{I}^{ \pm}$-canonical Frobenius splitting that is compatible with $\overline{\mathbb{O}(w)} s$ and $\overline{\mathbb{O}^{-}(v)} s\left(w, v \in W_{\mathrm{af}}\right)$.

2. (Corollary 4.10 and Remark 4.13) For each $w, v \in W_{\mathrm{af}}$, the intersection $\mathbb{Q}(v, w):=\overline{\mathbb{O}(w)} \cap \overline{\mathbb{O}^{-}(v)}$ is reduced. It is irreducible when $v=w_{0} t_{\beta}$ for some $\beta \in Q^{\vee}$.

3. (Lemma 4.7 and Corollary 5.24) For each $w, v \in W_{\mathrm{af}}$, the scheme $Q(v, w)$ is weakly normal. It is normal (and irreducible) when char $\mathbb{K}=0$ or char $\mathbb{K} \gg 0$. 
4. (Lemma 4.28) For each $\beta \in Q_{+}^{\vee}$, the set of $\mathbb{K}$-valued points of the scheme $\mathbb{Q}\left(w_{0} t_{\beta}, e\right)$ is in bijection with the set of ( $\mathbb{K}$-valued) Drinfeld-Plücker data. In particular, $\mathbb{Q}\left(w_{0} t_{\beta}, e\right)$ is isomorphic to the quasi-map space in [29] when $\mathbb{K}=\mathbb{C}$.

Theorem B is a key result at the deepest part (correspondence between natural bases) in our proof ([47]) of a conjecture of Lam, Li, Mihalcea and Shimozono [60] about the comparison between the equivariant $K$-group of the affine Grassmannian of $G$ and the equivariant small quantum $K$-group of $\mathscr{B}$. In [47], we also prove that $\mathscr{Q}\left(w_{0} t_{\beta}, w\right)$ admits only rational singularities (and hence it is CohenMacaulay) when $\mathbb{K}=\mathbb{C}$ on the basis of Theorem $\mathrm{B}$. We remark that Theorem $\mathrm{B}(3)$ is proved in $[9,10]$ when $v=w_{0} t_{\beta}, w=e$ and $\mathbb{K}=\mathbb{C}$.

Our proof of Theorem $\mathrm{B}(1)$ is not at all standard, and in fact it forms the core of the technical contributions in this paper. To appreciate its contents, let us recall that there are two standard ways to construct a Frobenius splitting of $\mathscr{B}$ ([14]): One is to consider the Bott-Samelson-Demazure-Hansen resolution of $\mathscr{B}$, which reduces the assertion to the case of a point, which is a Schubert variety with trivial Frobenius splitting. The other is to analyse the space of global sections of (some power of) the canonical bundle of $\mathscr{B}$.

However, neither of these proof strategies works for $\mathbf{Q}_{G}^{\text {rat }}$. The first one fails because any Schubert variety of $\mathbf{Q}_{G}^{\text {rat }}$ is infinite-dimensional, and carries rich internal structure by itself. The second one fails because the canonical bundle of $\mathbf{Q}_{G}^{\text {rat }}$ simply does not make sense, at least naively. These require some new ideas to prove Theorem B(1). Our idea here is as follows: An interpretation of the filtrations in [50] reduces the existence of a Frobenius splitting of $\mathbf{Q}_{G}^{\text {rat }}$ to a property of the Frobenius splitting of the corresponding thick affine flag manifold [49, Corollary B]. This property can be seen as a special case of some homological property in the representation theory of affine Lie algebras $([18,20])$, but it is proved only for characteristic 0 . Thus, we use Kashiwara's theory of global basis ([43, 44]) to transfer such a homological property into the positive-characteristic setting (Proposition 3.19).

In the rest of this introduction, we assume $\mathbb{K}=\mathbb{C}$ for the sake of simplicity. Let $P$ be the weight lattice of $H$, and let $P_{+} \subset P$ denote its subset corresponding to dominant weights. For each $\lambda \in P$, we have an equivariant line bundle $\mathcal{O}_{\mathbf{Q}_{G}^{\text {rat }}}(\lambda)$ on $\mathbf{Q}_{G}^{\text {rat }}$, whose restriction to $Q(v, w)$ is denoted by $\mathcal{O}_{Q}(v, w)(\lambda)$. Associated to $\lambda \in P_{+}$, we have a level 0 extremal weight module $\mathbb{X}(\lambda)$ of $U(\widetilde{\mathfrak{g}})$ in the sense of Kashiwara [44]. We know that $\mathbb{X}(\lambda)$ is equipped with two kinds of Demazure modules and a distinguished basis (the global basis).

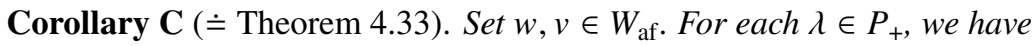

$$
H^{>0}\left(Q(v, w), \mathcal{O}_{Q}(v, w)(\lambda)\right)=\{0\} .
$$

The space $H^{0}\left(Q(v, w), \mathcal{O}_{Q}(v, w)(\lambda)\right)^{\vee}$ is the intersection of two Demazure modules of $\mathbb{X}(\lambda)$ spanned by a subset of the global basis of $\mathbb{X}(\lambda)$ if $\lambda$ is strictly dominant. If we have $w^{\prime}, v^{\prime} \in W_{\mathrm{af}}$ such that $\mathbb{Q}\left(v^{\prime}, w^{\prime}\right) \subset \mathbb{Q}(v, w)$, then the restriction map

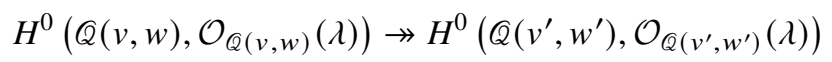

is surjective.

Note that Corollary $\mathrm{C}$ adds new vanishing region to [10, Theorem 3.1 1)]. We also provide parabolic versions of Theorems $\mathrm{A}$ and $\mathrm{B}$ and Corollary C. We have a description of $H^{0}\left(\mathcal{Q}(v, w), \mathcal{O}_{\mathbb{Q}(v, w)}(\lambda)\right)$ for general $\lambda \in P_{+}$that is more complicated (Theorem B.6).

Let $\mathscr{B}_{2, \beta}$ be the space of genus 0 stable maps with two marked points to $\mathscr{B}$ with the class of its image $\beta \in Q_{+}^{\vee} \subset Q^{\vee} \cong H_{2}(\mathscr{B}, \mathbb{Z})$. We have evaluation maps $\mathrm{e}_{j}: \mathscr{B}_{2, \beta} \rightarrow \mathscr{B}$ for $j=1,2$. The following purely geometric result is a by-product of our proof that may be of independent interest:

Corollary $\mathbf{D}$ ( $\doteq$ Corollary 5.19). Set $\beta \in Q_{+}^{\vee}$ and $x, y \in \mathscr{B}$. The space $\left(\mathrm{e}_{1}^{-1}(x) \cap \mathrm{e}_{2}^{-1}(\overline{B y})\right)$ is connected if it is nonempty. 
Note that Corollary D is contained in [16] whenever $\{x\}, \overline{B y} \subset \mathscr{B}$ are in general position.

The plan of this paper is as follows: In Section 2, we collect basic material needed in what follows. In Section 3, after recalling generalities on Frobenius splitting and representation theory of quantum loop algebras, we construct the ind-scheme $\mathbf{Q}_{G}^{\text {rat }}$ and equip it with a Frobenius splitting (Corollary 3.38). In Section 4, we first interpret $\mathbf{Q}_{G}^{\text {rat }}$ as an ind-scheme (coarsely) representing the coset $G(\mathbb{K}((z))) /(H(\mathbb{K}) N(\mathbb{K}((z))))$ (Theorem A). Using this, we identify some Richardson varieties of $\mathbf{Q}_{G}^{\text {rat }}$ with quasi-map spaces (Theorem 4.30) and present their cohomological properties (Theorem 4.33), and hence prove (large parts of) Theorem B and Corollary C. Since our construction equips quasi-map spaces with Frobenius splittings (Lemma 4.7), they are automatically weakly normal. Moreover, we explain how to connect characteristic 0 and positive characteristic (Section 4.5). In Section 5, we analyse the fibres of the graph-space resolutions of quasi-map spaces and deduce that Richardson varieties of semi-infinite flag manifolds (over $\mathbb{C}$ ) are normal based on the weak normality proved in the previous section. This proves the remaining part of Theorem B. Our analysis here contains an inductive proof that the fibres of the evaluation maps of the space of genus 0 stable maps to flag varieties are connected (Corollary D). In Appendix A, we give a proof of the normality of (the ind-pieces of) $\mathbf{Q}_{G}^{\text {rat }}$ (which works also in the positive characteristic setting) and present an analogue of the Kempf vanishing theorem [52] for $\mathbf{Q}_{G}^{\text {rat }}$. Appendix B exhibits the structure of global sections of nef line bundles of Richardson varieties of $\mathbf{Q}_{G}^{\text {rat }}$.

Note that Theorem B equips a quasi-map space from $\mathbb{P}^{1}$ to $\mathscr{B}$ with a Frobenius splitting compatible with the boundaries. However, the notion of a boundary in quasi-map spaces depends on a configuration of points in $\mathbb{P}^{1}$ (we implicitly set them to $\{0, \infty\} \subset \mathbb{P}^{1}$ throughout this paper). This makes our analogues of open Richardson varieties not necessarily smooth, contrary to the original case [76] (see also [29, $\S 8.4 .1])$. We hope to give a further account of this, as well as the factorisation structure ([29, §6.3]) from the viewpoint presented in this paper, in future work.

\section{Preliminaries}

We work over an algebraically closed field $\mathbb{K}$ unless stated otherwise. A vector space is a $\mathbb{K}$-vector space, and a graded vector space refers to a $\mathbb{Z}$-graded vector space whose graded pieces are finite-dimensional and whose grading is bounded from above or from below. Tensor products are taken over $\mathbb{K}$ unless specified otherwise.

Let $A$ be a principal ideal domain. For a graded free $A$-module $M=\bigoplus_{m \in \mathbb{Z}} M_{m}$, we set $M^{\vee}:=$ $\bigoplus_{m \in \mathbb{Z}} \operatorname{Hom}_{A}\left(M_{m}, A\right)$, where $\operatorname{Hom}_{A}\left(M_{m}, A\right)$ is understood to have degree $-m$.

As a rule, we suppress $\emptyset$ and associated parentheses from notation. This particularly applies to $\emptyset=\mathrm{J} \subset \mathrm{I}$, frequently used to specify parabolic subgroups.

\subsection{Groups, root systems and Weyl groups}

We refer to $[21,57]$ for precise expositions of general material presented in this subsection.

Let $G$ be a connected, simply connected simple algebraic group of rank $r$ over an algebraically closed field $\mathbb{K}$, and let $B$ and $H$ be a Borel subgroup and a maximal torus of $G$ such that $H \subset B$. We set $N(=[B, B])$ to be the unipotent radical of $B$ and let $N^{-}$be the opposite unipotent subgroup of $N$ with respect to $H$. We denote the Lie algebra of an algebraic group by the corresponding German (Fraktur) small letter. We have a (finite) Weyl group $W:=N_{G}(H) / H$. For an algebraic group $E$, we denote its set of $\mathbb{K}[z]$-valued points by $E[z]$, its set of $\mathbb{K}[[z]]$-valued points by $E[[z]]$, and its set of $\mathbb{K}((z))$-valued points by $E((z))$, and so on. Let $\mathbf{I} \subset G[[z]]$ be the preimage of $B \subset G$ via the evaluation at $z=0$ (the Iwahori subgroup of $G[[z]])$. We set $\mathbf{I}^{-} \subset G\left[z^{-1}\right]$ as the opposite Iwahori subgroup of $\mathbf{I}$ in $G((z))$ with respect to $H$. By abuse of notation, we might consider $\mathbf{I}$ and $G[[z]]$ as proalgebraic groups over $\mathbb{K}$ whose $\mathbb{K}$-valued points are given as these.

Let $P:=\operatorname{Hom}_{g r}\left(H, \mathbb{G}_{m}\right)$ be the weight lattice of $H, \Delta \subset P$ be the set of roots, $\Delta_{+} \subset \Delta$ be the set of roots that yield root subspaces in $\mathfrak{b}$ and $\Pi \subset \Delta_{+}$be the set of simple roots. We set $\Delta_{-}:=-\Delta_{+}$. Let 
$Q^{\vee}$ be the dual lattice of $P$ with a natural pairing $\langle\bullet, \bullet\rangle: Q^{\vee} \times P \rightarrow \mathbb{Z}$. We define $\Pi^{\vee} \subset Q^{\vee}$ to be the set of positive simple coroots and let $Q_{+}^{\vee} \subset Q^{\vee}$ be the set of nonnegative integer spans of $\Pi^{\vee}$. For $\beta, \gamma \in Q^{\vee}$, we define $\beta \geq \gamma$ if and only if $\beta-\gamma \in Q_{+}^{\vee}$. We set $P_{+}:=\left\{\lambda \in P \mid\left\langle\alpha^{\vee}, \lambda\right\rangle \geq 0, \forall \alpha^{\vee} \in \Pi^{\vee}\right\}$ and $P_{++}:=\left\{\lambda \in P \mid\left\langle\alpha^{\vee}, \lambda\right\rangle>0, \forall \alpha^{\vee} \in \Pi^{\vee}\right\}$. Define $\mathrm{I}:=\{1,2, \ldots, r\}$. We fix bijections $\mathrm{I} \cong \Pi \cong \Pi^{\vee}$ such that $i \in \mathrm{I}$ corresponds to $\alpha_{i} \in \Pi$, its coroot $\alpha_{i}^{\vee} \in \Pi^{\vee}$ and a simple reflection $s_{i} \in W$ corresponding to $\alpha_{i}$. Let $\left\{\varpi_{i}\right\}_{i \in \mathrm{I}} \subset P_{+}$be the set of fundamental weights (i.e., $\left.\left\langle\alpha_{i}^{\vee}, \varpi_{j}\right\rangle=\delta_{i j}\right)$ and $\rho:=\sum_{i \in \mathrm{I}} \varpi_{i}=$ $\frac{1}{2} \sum_{\alpha \in \Delta^{+}} \alpha \in P_{+}$.

For a subset $\mathrm{J} \subset \mathrm{I}$, we define $P(\mathrm{~J})$ as the standard parabolic subgroup of $G$ corresponding to $\mathrm{J}$ - that is, we have $\mathfrak{b} \subset \mathfrak{p}(J) \subset \mathfrak{g}$, and $\mathfrak{p}(J)$ contains the root subspace corresponding to $-\alpha_{i}(i \in \mathrm{I})$ if and only if $i \in \mathrm{J}$. Let $H \subset L(\mathrm{~J}) \subset P(\mathrm{~J})$ be the standard Levi subgroup (which is isomorphic to the quotient of $P(\mathrm{~J}$ ) by its unipotent radical). Then the set of characters of $P(\mathrm{~J})$ is identified with $P_{\mathrm{J}}:=\sum_{i \in \mathrm{I} \backslash \mathrm{J}} \mathbb{Z} \varpi_{i}$. We also set $P_{\mathrm{J},+}:=\sum_{i \in \mathrm{I} \backslash \mathrm{J}} \mathbb{Z}_{\geq 0} \varpi_{i}=P_{+} \cap P_{\mathrm{J}}$ and $P_{\mathrm{J},++}:=\sum_{i \in \mathrm{I} \backslash \mathrm{J}} \mathbb{Z}_{\geq 1} \varpi_{i}=P_{++} \cap P_{\mathrm{J}}$. We define $W_{\mathrm{J}} \subset W$ to be the reflection subgroup generated by $\left\{s_{i}\right\}_{i \in \mathrm{J}}$. It is the Weyl group of $[L(\mathrm{~J}), L(\mathrm{~J})]$ and $L(\mathrm{~J})$. We define $\rho_{\mathrm{J}}$ to be the half-sum of positive roots whose root spaces are contained in the unipotent radical of $\mathfrak{p}(\mathrm{J})$.

Let $\Delta_{\text {af }}:=\Delta \times \mathbb{Z} \delta \cup\{m \delta\}_{m \neq 0}$ be the untwisted affine root system of $\Delta$ with its positive part $\Delta_{+} \subset \Delta_{\text {af, }+}$. We set $\alpha_{0}:=-\vartheta+\delta, \Pi_{\mathrm{af}}:=\Pi \cup\left\{\alpha_{0}\right\}$ and $\mathrm{I}_{\mathrm{af}}:=\mathrm{I} \cup\{0\}$, where $\vartheta$ is the highest root of $\Delta_{+}$. We set $W_{\text {af }}:=W \ltimes Q^{\vee}$ and call it the affine Weyl group. It is a reflection group generated by $\left\{s_{i} \mid i \in \mathrm{I}_{\mathrm{af}}\right\}$, where $s_{0}$ is the reflection with respect to $\alpha_{0}$. We also have a reflection $s_{\alpha} \in W_{\text {af }}$ corresponding to $\alpha \in \Delta \times \mathbb{Z} \delta \subsetneq \Delta^{\text {af }}$. Let $\ell: W_{\text {af }} \rightarrow \mathbb{Z}_{\geq 0}$ be the length function and $w_{0} \in W$ be the longest element in $W \subset W_{\text {af }}$. Together with the normalisation $t_{-\vartheta^{\vee}}:=s_{\vartheta} s_{0}$ (for the coroot $\vartheta^{\vee}$ of $\vartheta$ ), we introduce the translation element $t_{\beta} \in W_{\text {af }}$ for each $\beta \in Q^{\vee}$.

For each $i \in \mathrm{I}_{\mathrm{af}}$, we have a connected algebraic group $\operatorname{SL}(2, i)$ that is isomorphic to $S L(2)$ equipped with an inclusion $S L(2, i)(\mathbb{K}) \subset G((z))$ as groups corresponding to $\pm \alpha_{i} \in \mathrm{I}_{\text {af }}$. Let $\rho_{ \pm \alpha_{i}}: \mathbb{G}_{m} \rightarrow S L(2, i)$ denote the unipotent one-parameter subgroup corresponding to $\pm \alpha_{i} \in \Delta_{\text {af }}$. We set $B_{i}:=S L(2, i) \cap \mathbf{I}$, which is a Borel subgroup of $S L(2, i)$. For each $i \in \mathrm{I}$, we set $P_{i}:=P(\{i\})$. For each $i \in \mathrm{I}_{\mathrm{af}}$, we set $\mathbf{I}(i):=S L(2, i) \mathbf{I}$. Each $\mathbf{I}(i)$ can be regarded as a proalgebraic group.

As a variation of [57, Chapter VI], we say an ind-scheme $\mathfrak{X}$ over $\mathbb{K}$ admits a $G((z))$-action if it admits an action of $\mathbf{I}$ and $S L(2, i)\left(i \in \mathrm{I}_{\mathrm{af}}\right)$ as (ind-)schemes over $\mathbb{K}$ that coincides on $B_{i}=(\mathbf{I} \cap S L(2, i))$ and they generate a $G((z))$-action on the set of closed points of $\mathfrak{X}$ (the latter is a group action on a set). We consider the notion of $G((z))$-equivariant morphisms accordingly.

We set

$$
Q_{<}^{\vee}:=\left\{\beta \in Q^{\vee} \mid\left\langle\beta, \alpha_{i}\right\rangle<0, \forall i \in \mathrm{I}\right\} .
$$

Let $\leq$ be the Bruhat order of $W_{\mathrm{af}}$. In other words, $w \leq v$ holds if and only if a subexpression of a reduced decomposition of $v$ yields a reduced decomposition of $w$. We define the generic (semi-infinite) Bruhat order $\leq \frac{\infty}{2}$ as

$$
w \leq_{\frac{\infty}{2}} v \Leftrightarrow w t_{\beta} \leq v t_{\beta} \quad \text { for every } \beta \in Q^{\vee} \text { such that }\left\langle\beta, \alpha_{i}\right\rangle \ll 0 \text { for } i \in \mathrm{I} .
$$

By [64], this defines a preorder on $W_{\text {af }}$. Here we remark that $w \leq v$ if and only if $w \geq \frac{\infty}{2} v$ for $w, v \in W$. We also have

$$
w \leq_{\frac{\infty}{2}} v \Leftrightarrow w w_{0} \geq_{\frac{\infty}{2}} v w_{0}, \quad w, v \in W_{\mathrm{af}} .
$$

For proofs and related results, we refer to [51, §2.2] and [74, Lecture 13].

For each $u \in W$ and $\beta \in Q^{\vee}$, we set

$$
\ell^{\frac{\infty}{2}}\left(u t_{\beta}\right):=\ell(u)+\sum_{\alpha \in \Delta_{+}}\langle\beta, \alpha\rangle=\ell(u)+2\langle\beta, \rho\rangle .
$$

Theorem 2.1 ([64]; compare [61]). For each $w, v \in W_{\mathrm{af}}$ such that $w \leq \frac{\infty}{2} v$, there exists $\alpha \in \Delta_{+}^{\mathrm{af}}$ such that $w \leq_{\frac{\infty}{2}} s_{\alpha} v \leq_{\frac{\infty}{2}} v$ and $\ell^{\frac{\infty}{2}}\left(s_{\alpha} v\right)=\ell^{\frac{\infty}{2}}(v)+1$. 
For each $\lambda \in P_{+}$, we denote the corresponding Weyl module by $V(\lambda)$ (see, e.g. [1, Proposition 1.22] and [41, Theorem 5]). By convention, $V(\lambda)$ is a finite-dimensional indecomposable $G$-module with a cyclic $B$-eigenvector $\mathbf{v}_{\lambda}^{0}$ (highest weight vector) with $H$-weight $\lambda$ whose character obeys the Weyl character formula. For a semisimple $H$-module $V$, we set

$$
\operatorname{ch} V:=\sum_{\lambda \in P} e^{\lambda} \cdot \operatorname{dim}_{\mathbb{K}} \operatorname{Hom}_{H}\left(\mathbb{K}_{\lambda}, V\right) .
$$

If $V$ is a $\mathbb{Z}$-graded $H$-module in addition, then we set

$$
\operatorname{gch} V:=\sum_{\lambda \in P, n \in \mathbb{Z}} q^{n} e^{\lambda} \cdot \operatorname{dim}_{\mathbb{K}} \operatorname{Hom}_{H}\left(\mathbb{K}_{\lambda}, V_{n}\right)
$$

Define $\mathscr{B}:=G / B$ and call it the flag manifold of $G$. We have the Bruhat decomposition

$$
\mathscr{B}=\bigsqcup_{w \in W} \mathbb{O}_{\mathscr{B}}(w)
$$

into $B$-orbits such that $\operatorname{dim} \mathbb{O}_{\mathscr{B}}(w)=\ell\left(w_{0}\right)-\ell(w)$ for each $w \in W \subset W_{\text {af }}$. We set $\mathscr{B}(w):=\overline{\mathbb{O}_{\mathscr{B}}(w)} \subset$ $\mathscr{B}$.

For each $\lambda \in P$, we have a line bundle $\mathcal{O}_{\mathscr{B}}(\lambda)$ such that

$$
H^{0}\left(\mathscr{B}, \mathcal{O}_{\mathscr{B}}(\lambda)\right) \cong V(\lambda)^{*}, \quad \mathcal{O}_{\mathscr{B}}(\lambda) \otimes_{\mathcal{O}_{\mathscr{B}}} \mathcal{O}_{\mathscr{B}}(-\mu) \cong \mathcal{O}_{\mathscr{B}}(\lambda-\mu), \quad \lambda, \mu \in P_{+}
$$

For each $w \in W$, let $p_{w} \in \mathbb{O}_{\mathscr{B}}(w)$ be the unique $H$-fixed point. We normalise $p_{w}$ (and hence $\mathbb{O}_{\mathscr{B}}(w)$ ) so that the restriction of $\mathcal{O}_{\mathscr{B}}(\lambda)$ to $p_{w}$ is isomorphic to $\mathbb{K}_{-w w_{0} \lambda}$ for every $\lambda \in P_{+}$. (Here we warn that the convention differs from [47].)

\subsection{Representations of affine and current algebras}

In the rest of this section, we work over $\mathbb{K}=\mathbb{C}$, the field of complex numbers. Material in this subsection without a reference can be found in $[40,41]$. Every result in this subsection is transferred to an arbitrary field in Section 3.2.

Let $\widetilde{\mathfrak{g}}$ denote the untwisted affine Kac-Moody algebra associated to $\mathfrak{g}-$ that is, we have

$$
\widetilde{\mathfrak{g}}=\mathfrak{g} \otimes \mathbb{C}\left[z, z^{-1}\right] \oplus \mathbb{C} K \oplus \mathbb{C} d,
$$

where $K$ is central, $\left[d, X \otimes z^{m}\right]=m X \otimes z^{m}$ for each $X \in \mathfrak{g}$ and $m \in \mathbb{Z}$ and, for each $X, Y \in \mathfrak{g}$ and $f, g \in \mathbb{C}\left[z^{ \pm 1}\right]$, we have

$$
[X \otimes f, Y \otimes g]=[X, Y] \otimes f g+(X, Y)_{\mathfrak{g}} \cdot K \cdot \operatorname{Res}_{z=0} f \frac{\partial g}{\partial z}
$$

where $(\bullet, \bullet)_{\mathfrak{g}}$ denotes the $G$-invariant bilinear form such that $\left(\alpha^{\vee}, \alpha^{\vee}\right)_{\mathfrak{g}}=2$ for a long simple root $\alpha$. Let $E_{i}, F_{i}\left(i \in \mathrm{I}_{\mathrm{af}}\right)$ denote the Kac-Moody generators of $\widetilde{\mathfrak{g}}$ corresponding to $\alpha_{i}$. We set $\widetilde{\mathfrak{h}}:=\mathfrak{h} \oplus \mathbb{C} K \oplus \mathbb{C} d$. Let $\mathfrak{I}$ be the Lie subalgebra of $\widetilde{\mathfrak{g}}$ generated by $E_{i}\left(i \in \mathrm{I}_{\mathrm{af}}\right)$ and $\widetilde{\mathfrak{h}}$, and $\mathfrak{I}^{-}$be the Lie subalgebra of $\widetilde{\mathfrak{g}}$ generated by $F_{i}\left(i \in \mathrm{I}_{\mathrm{af}}\right)$ and $\widetilde{\mathfrak{h}}$. For each $i \in \mathrm{I}_{\mathrm{af}}$ and $n \geq 0$, we set $E_{i}^{(n)}:=\frac{1}{n !} E_{i}^{n}$ and $F_{i}^{(n)}:=\frac{1}{n !} F_{i}^{n}$.

We define

$$
Q^{\mathrm{af}, \vee}:=\mathbb{Z} d \oplus \bigoplus_{i \in \mathrm{I}_{\mathrm{af}}} \mathbb{Z} \alpha_{i}^{\vee} \subset \widetilde{\mathfrak{h}}, \quad P^{\mathrm{af}}:=\mathbb{Z} \delta \oplus \bigoplus_{i \in \mathrm{I}_{\mathrm{af}}} \mathbb{Z} \Lambda_{i} \subset \widetilde{\mathfrak{h}}^{*},
$$


and a pairing $Q^{\mathrm{af}, \vee} \times P^{\text {af }} \rightarrow \mathbb{Z}$ such that

$$
\left\langle\alpha_{i}^{\vee}, \Lambda_{j}\right\rangle=\delta_{i j} \quad\left(i, j \in \mathrm{I}_{\mathrm{af}}\right), \quad\left\langle\alpha_{i}^{\vee}, \delta\right\rangle \equiv 0, \quad\left\langle d, \Lambda_{i}\right\rangle=\delta_{i 0} \quad\left(i \in \mathrm{I}_{\mathrm{af}}\right), \quad\langle d, \delta\rangle=1 .
$$

We have a projection map

$$
P^{\mathrm{af}} \ni \Lambda=k \delta+\sum_{i \in \mathrm{I}_{\mathrm{af}}} a_{i} \Lambda_{i} \mapsto \bar{\Lambda}=\sum_{i \in \mathrm{I}} a_{i} \varpi_{i} \in P,
$$

which has a unique splitting $P \subset P^{\text {af }}$ whose image is orthogonal to $d, K \in \widetilde{\mathfrak{h}}$. We set $P_{+}^{\text {af }}:=\sum_{i \in \mathrm{I}_{\mathrm{af}}} \mathbb{Z}_{\geq 0} \Lambda_{i}$. Each $\Lambda \in P_{+}^{\text {af }}$ defines an irreducible integrable highest weight module $L(\Lambda)$ of $\widetilde{\mathfrak{g}}$ with its highest weight vector $\mathbf{v}_{\Lambda}$. In addition, each $\lambda \in P_{+}$defines a level 0 extremal weight module $\mathbb{X}(\lambda)$ of $\widetilde{\mathfrak{g}}$ by means of the specialisation of the quantum parameter $q=1$ in [43, Proposition 8.2.2] and [44, §5.1], which is integrable, and $K$ acts by 0 . The module $\mathbb{X}(\lambda)$ carries a cyclic $\mathfrak{h}$-weight vector $\mathbf{v}_{\lambda}$ such that

$$
H \mathbf{v}_{\lambda}=\lambda(H) \mathbf{v}_{\lambda}(H \in \mathfrak{h}), \quad K \mathbf{v}_{\lambda}=0=d \mathbf{v}_{\lambda}, \quad E_{i} \mathbf{v}_{\lambda}=0 \quad(i \in \mathrm{I}), \text { and } F_{0} \mathbf{v}_{\lambda}=0 .
$$

(We can deduce that $\mathbb{X}(\lambda)$ is the maximal integrable $\widetilde{\mathfrak{g}}$-module that possesses a cyclic vector with these properties [43, §8.1].) Moreover, each $w=u t_{\beta} \in W_{\mathrm{af}}\left(u \in W, \beta \in Q^{\vee}\right)$ defines an element $\mathbf{v}_{w \lambda} \in \mathbb{X}(\lambda)$ such that

$$
H \mathbf{v}_{w \lambda}=(w \lambda)(H) \mathbf{v}_{w \lambda} \quad(H \in \mathfrak{h}), \quad K \mathbf{v}_{w \lambda}=0, \quad d \mathbf{v}_{w \lambda}=-\langle\beta, \lambda\rangle \mathbf{v}_{w \lambda}
$$

up to sign $[43, \S 8.1]$. We call a vector in $\left\{\mathbf{v}_{w \lambda}\right\}_{w \in W_{\text {af }}}$ an extremal weight vector of $\mathbb{X}(\lambda)$.

We set $\mathfrak{g}[z]:=\mathfrak{g} \otimes_{\mathbb{C}} \mathbb{C}[z]$ and regard it as a Lie subalgebra of $\widetilde{\mathfrak{g}}$. We have $\mathfrak{I} \subset \mathfrak{g}[z]+\mathbb{C} K+\mathbb{C} d$. The Lie algebra $\mathfrak{g}[z]$ is graded, and its grading is the internal grading of $\widetilde{\mathfrak{g}}$ given by $d$.

For each $\lambda \in P_{+}$, we set

$$
\mathbb{W}_{w}(\lambda):=U(\mathfrak{I}) \mathbf{v}_{w \lambda} \subset \mathbb{X}(\lambda)
$$

These are the $q=1$ cases of the Demazure modules of $\mathbb{X}(\lambda)$, as well as the generalised global Weyl modules in the sense of [28]. We set $\mathbb{W}(\lambda):=\mathbb{W}_{w_{0}}(\lambda)$. By construction, both $\mathbb{X}(\lambda)$ and $\mathbb{W}_{w}(\lambda)$ are semisimple as $\left(H \times \mathbb{G}_{m}\right)$-modules, where $\mathbb{G}_{m}$ acts on $z$ by $a: z^{m} \mapsto a^{m} z^{m}(m \in \mathbb{Z})$.

Theorem 2.2 ([62]; compare [46]). For each $\lambda \in P_{+}$, the $\mathfrak{I}$-action on $\mathbb{W}(\lambda)$ prolongs to $\mathfrak{g}[z]$ and is isomorphic to the global Weyl module of $\mathfrak{g}[z]$ in the sense of Chari and Pressley [19]. Moreover, $\mathbb{W}(\lambda)$ is a projective module in the category of $\mathfrak{g}[z]$-modules whose restriction to $\mathfrak{g}$ is a direct sum of modules in $\{V(\mu)\}_{\mu \leq \lambda}$.

Theorem 2.3 ([46]). Set $\lambda, \mu \in P_{+}$and $w \in W$. We have a unique (up to scalar) injective degree 0 I-module map

$$
\mathbb{W}_{w}(\lambda+\mu) \hookrightarrow \mathbb{W}_{w}(\lambda) \otimes \mathbb{W}_{w}(\mu)
$$

Sketch of proof. For each $\lambda, \mu \in P_{+}$, the projectivity of $\mathbb{W}(\lambda+\mu)$ in the sense of Theorem 2.2 yields a unique graded $\mathfrak{g}[z]$-module map

$$
\mathbb{W}(\lambda+\mu) \longrightarrow \mathbb{W}(\lambda) \otimes \mathbb{W}(\mu)
$$

of degree 0 . This map is injective by examining the specialisations to local Weyl modules (for their definitions, see [46, Theorem 1.4] or Lemma 3.20 and Remark 3.21). Examining the $\mathfrak{I}$-cyclic vectors, it uniquely restricts to a map

$$
\mathbb{W}_{w}(\lambda+\mu) \longrightarrow \mathbb{W}_{w}(\lambda) \otimes \mathbb{W}_{w}(\mu)
$$

up to scalar. Because the ambient map is injective, this map must be also. 


\subsection{Semi-infinite flag manifolds}

We work over $\mathbb{C}$ as in the previous subsection. Material in this section is re-proved in the setting of characteristic $\neq 2$ in Sections 3.4 and 4.2 (compare Section 4.3). We define the semi-infinite flag manifold as the reduced ind-scheme such that both of the following are true:

- We have a closed embedding

$$
\mathbf{Q}_{G}^{\mathrm{rat}} \subset \prod_{i \in \mathrm{I}} \mathbb{P}\left(V\left(\varpi_{i}\right) \otimes \mathbb{C}((z))\right)
$$

○ We have an equality $\mathbf{Q}_{G}^{\text {rat }}(\mathbb{C})=G((z)) /(H(\mathbb{C}) \cdot N((z)))$.

This is a pure ind-scheme of ind-infinite type [51]. Note that the group $Q^{\vee} \subset H((z)) / H(\mathbb{C})$ acts on $\mathbf{Q}_{G}^{\text {rat }}$ from the right. The ind-scheme $\mathbf{Q}_{G}^{\text {rat }}$ is equipped with a $G((z))$-equivariant line bundle $\mathcal{O}_{\mathbf{Q}_{G}^{\text {rat }}}(\lambda)$ for each $\lambda \in P$. Here we normalised so that $\Gamma\left(\mathbf{Q}_{G}^{\text {rat }}, \mathcal{O}_{\mathbf{Q}_{G}^{\text {rat }}}(\lambda)\right)$ is $B^{-}((z))$-cocyclic to an $H$-weight vector with its $H$-weight $-\lambda$. We warn that this convention is twisted by $-w_{0}$ from that of [47], and complies with [51].

Theorem 2.4 ([29, 23, 51, 64]). We have an I-orbit decomposition

$$
\mathbf{Q}_{G}^{\mathrm{rat}}=\bigsqcup_{w \in W_{\mathrm{af}}} \mathcal{O}(w)
$$

with the following properties:

1. Each $\mathbb{O}(w)$ is isomorphic to $\mathbb{A}^{\infty}$ and has a unique $\left(H \times \mathbb{G}_{m}\right)$-fixed point.

2. The right action of $\gamma \in Q^{\vee}$ on $\mathbf{Q}_{G}^{\text {rat }}$ yields the translation $\mathbb{O}(w) \mapsto \mathbb{O}\left(w t_{\gamma}\right)$.

3. We have $\mathbb{O}(w) \subset \overline{\mathbb{O}(v)}$ if and only if $w \leq \frac{\infty}{2} v$.

4. The relative dimension of $\mathbb{O}\left(u t_{\beta}\right)\left(u \in W, \beta \in Q^{\vee}\right)$ and $\mathbb{O}(e)$, counted as the difference of the cardinality of the maximal chain of intermediate $\mathbf{I}$-orbits to a common smaller $\mathbf{I}$-orbit, is $\ell^{\frac{\infty}{2}}\left(u t_{\beta}\right)$.

For each $w \in W_{\text {af }}$, let $\mathbf{Q}_{G}(w)$ denote the closure of $\mathbb{O}(w)$. We refer to $\mathbf{Q}_{G}(w)$ as a Schubert variety of $\mathbf{Q}_{G}^{\text {rat }}$ (corresponding to $w \in W_{\text {af }}$ ).

Let $S=\bigoplus_{\lambda \in P_{\mathrm{J},+}} S(\lambda)$ be a $P_{\mathrm{J}^{+}-\text {graded commutative ring such that }} S(0)=A$ is a principal ideal domain and $S$ is torsion-free over $A$ and generated by $\bigoplus_{i \in \mathrm{I} \backslash \mathrm{J}} S\left(\varpi_{i}\right)$. We define

$$
\operatorname{Proj} S=(\operatorname{Spec} S \backslash E) / H \subset \prod_{i \in \mathrm{I} \backslash \mathrm{J}} \mathbb{P}_{A}\left(S\left(\varpi_{i}\right)^{\vee}\right)
$$

as the $P_{\mathrm{J},+}$-graded proj over $\operatorname{Spec} A$, where $E$ is the locus where all of $S\left(\varpi_{i}\right)$ vanishes for some $i \in \mathrm{I} \backslash \mathrm{J}$ (the irrelevant locus).

Theorem 2.5 ([51]). For each $w \in W_{\text {af }}$, we have

$$
\mathbf{Q}_{G}(w) \cong \operatorname{Proj} \bigoplus_{\lambda \in P_{+}} \mathbb{W}_{w w_{0}}(\lambda)^{\vee},
$$

where the multiplication of the ring $\bigoplus_{\lambda} \mathbb{W}_{w w_{0}}(\lambda)^{\vee}$ is given by Theorem 2.3.

\subsection{Quasi-map spaces and Zastava spaces}

We work over $\mathbb{C}$ as in the previous subsection. Here we recall basics of quasi-map spaces from [29, 23].

We have $W$-equivariant isomorphisms $H^{2}(\mathscr{B}, \mathbb{Z}) \cong P$ and $H_{2}(\mathscr{B}, \mathbb{Z}) \cong Q^{\vee}$. This identifies the (integral points of the) nef cone of $\mathscr{B}$ with $P_{+} \subset P$ and the effective cone of $\mathscr{B}$ with $Q_{+}^{\vee}$. A quasi-map 
$(f, D)$ is a map $f: \mathbb{P}^{1} \rightarrow \mathscr{B}$ together with a $\Pi^{\vee}$-coloured effective divisor

$$
D=\sum_{\alpha \in \Pi^{\vee}, x \in \mathbb{P}^{1}(\mathbb{C})} m_{x}\left(\alpha^{\vee}\right) \alpha^{\vee} \otimes[x] \in Q^{\vee} \otimes_{\mathbb{Z}} \operatorname{Div}^{1}, \quad m_{x}\left(\alpha^{\vee}\right) \in \mathbb{Z}_{\geq 0} .
$$

For $i \in \mathrm{I}$, we set $D_{i}:=\left\langle D, \varpi_{i}\right\rangle \in \operatorname{Div} \mathbb{P}^{1}$. We call $D$ the defect of the quasi-map $(f, D)$. Here we define the (total) degree of the defect by

$$
|D|:=\sum_{\alpha \in \Pi^{\vee}, x \in \mathbb{P}^{1}(\mathbb{C})} m_{x}\left(\alpha^{\vee}\right) \alpha^{\vee} \in Q_{+}^{\vee} .
$$

For each $\beta \in Q_{+}^{\vee}$, we set

$$
\mathscr{Q}(\mathscr{B}, \beta):=\left\{f: \mathbb{P}^{1} \rightarrow X \mid \text { quasi-map such that } f_{*}\left[\mathbb{P}^{1}\right]+|D|=\beta\right\},
$$

where $f_{*}\left[\mathbb{P}^{1}\right]$ is the class of the image of $\mathbb{P}^{1}$ multiplied by the degree of $\mathbb{P}^{1} \rightarrow \operatorname{Im} f$. We denote $Q(\mathscr{B}, \beta)$ by $Q(\beta)$ when there is no danger of confusion.

Definition 2.6. (Drinfeld-Plücker data). Consider a collection $\mathcal{L}=\left\{\left(\psi_{\lambda}, \mathcal{L}^{\lambda}\right)\right\}_{\lambda \in P_{+}}$of inclusions $\psi_{\lambda}$ : $\mathcal{L}^{\lambda} \hookrightarrow V(\lambda) \otimes_{\mathbb{C}} \mathcal{O}_{\mathbb{P}^{1}}$ of line bundles $\mathcal{L}^{\lambda}$ over $\mathbb{P}^{1}$. The data $\mathcal{L}$ are called Drinfeld-Plücker data (DP-data) if the canonical inclusion of $G$-modules

$$
\eta_{\lambda, \mu}: V(\lambda+\mu) \hookrightarrow V(\lambda) \otimes V(\mu)
$$

induces an isomorphism

$$
\eta_{\lambda, \mu} \otimes \mathrm{id}: \psi_{\lambda+\mu}\left(\mathcal{L}^{\lambda+\mu}\right) \stackrel{\cong}{\longrightarrow} \psi_{\lambda}\left(\mathcal{L}^{\lambda}\right) \otimes_{\mathcal{O}_{\mathbb{P}}} \psi_{\mu}\left(\mathcal{L}^{\mu}\right)
$$

for every $\lambda, \mu \in P_{+}$.

Theorem 2.7 (Drinfeld; see [29]). The variety $Q(\beta)$ is isomorphic to the variety formed by isomorphism classes of the DP-data $\mathcal{L}=\left\{\left(\psi_{\lambda}, \mathcal{L}^{\lambda}\right)\right\}_{\lambda \in P_{+}}$such that $\operatorname{deg} \mathcal{L}^{\lambda}=\left\langle w_{0} \beta, \lambda\right\rangle$. In addition, $Q(\beta)$ is an irreducible variety of dimension $2\langle\rho, \beta\rangle+\ell\left(w_{0}\right)$.

For each $w \in W$, let $\mathscr{Z}(\beta, w) \subset \mathbb{Q}(\beta)$ be the locally closed subset consisting of quasi-maps that are defined at $z=0$, and their values at $z=0$ are contained in $\mathscr{B}(w) \subset \mathscr{B}$. We set $\mathscr{Q}(\beta, w):=\overline{\mathscr{Z}}(\beta, w)$. (Hence, we have $\mathbb{Q}(\beta)=\mathbb{Q}(\beta, e)$.)

Theorem 2.8 ([29]). Let $\mathbb{K}$ be an algebraically closed field (not necessarily of characteristic 0 ), and let $Q(\beta)_{\mathbb{K}}$ and $\mathscr{Z}\left(\beta, w_{0}\right)_{\mathbb{K}}$ be the spaces obtained by replacing the base field $\mathbb{C}$ with $\mathbb{K}$ in Definition 2.6. For each $\beta \in Q_{+}^{\vee}$, the space $\mathscr{Z}\left(\beta, w_{0}\right)_{\mathbb{K}}$ is an irreducible affine scheme equipped with an action of $\left(B \times \mathbb{G}_{m}\right)$ over $\mathbb{K}$. In addition, this action has a unique fixed point.

Remarks on the proof. Theorem 2.8 is proved in [29] for $\mathbb{K}=\mathbb{C}$ using [70], and in the current setting in [12] using [13]. One can also replace the usage of [70] with [81, Corollary 5.3.8] along the lines of [29].

For each $\lambda \in P$ and $w \in W$, we have a $G$-equivariant line bundle $\mathcal{O}_{Q}(\beta, w)(\lambda)$ (and its pro-object $\mathcal{O}_{Q}(\lambda)$ ) obtained by the (tensor products of the) pullbacks $\mathcal{O}_{Q}(\beta, w)\left(\varpi_{i}\right)$ of the $i$ th $\mathcal{O}(1)$ via the embedding

$$
\mathbb{Q}(\beta, w) \hookrightarrow \prod_{i \in \mathrm{I}} \mathbb{P}\left(V\left(\varpi_{i}\right) \otimes_{\mathbb{C}} \mathbb{C}[z]_{\leq-\left\langle w_{0} \beta, \varpi_{i}\right\rangle}\right)
$$

for each $\beta \in Q_{+}^{\vee}$. 
We have embeddings $\mathscr{B} \subset Q(\beta) \subset \mathbf{Q}_{G}(e)$ such that the line bundles $\mathcal{O}(\lambda)(\lambda \in P)$ correspond to each other by restrictions $([10,46,51])$.

\section{Semi-infinite flag manifolds over $\mathbb{Z}\left[\frac{1}{2}\right]$}

We keep the settings of the previous section. In this section, we sometimes work over a (commutative) ring or a non-algebraically closed field. For a ring $S$ or a scheme $\mathfrak{X}$, we may write $S_{A}$ or $\mathfrak{X}_{A}$ if it is defined over $A$. In addition, we may consider their scalar extensions $S_{B}:=S_{A} \otimes_{A} B$ and $\mathfrak{X}_{B}$ for a ring map $A \rightarrow B$.

\subsection{Frobenius splittings}

Let $\mathbb{k}$ be a field and $p$ be a prime. We assume char $\mathbb{k}=p, \mathbb{k} \subset \mathbb{K}$ and that the $p$ th power map is invertible on $\mathbb{k}\left(\right.$ e.g., $\mathbb{k}=\mathbb{F}_{p}$ or $\overline{\mathbb{F}}_{p}$ ) throughout this subsection.

We follow the generality on Frobenius splittings in [14], which considers separated schemes of finite type. We sometimes use the assertions from [14] without the finite-type assumption when the assertion is independent of that; typical disguises are properness, finite generation and the Serre vanishing theorem.

Definition 3.1. (Frobenius splitting of a ring). Let $R$ be a commutative ring over $\mathbb{k}$, and let $R^{(1)}$ denote the set $R$ equipped with the map

$$
R \times R^{(1)} \ni(r, m) \mapsto r^{p} m \in R^{(1)} .
$$

This equips $R^{(1)}$ with an $R$-module structure over $\mathbb{k}$ (the $\mathbb{k}$-vector space structure on $R^{(1)}$ is also twisted by the $p$ th power operation), together with a map $\imath: R .1 \rightarrow R^{(1)}$. An $R$-module map $\phi: R^{(1)} \rightarrow R$ is said to be a Frobenius splitting if $\phi \circ \imath$ is an identity.

Note that $l$ in Definition 3.1 must be an inclusion if we have a Frobenius splitting $\phi$. Since the $p$ th power map in $\mathbb{k}$ is invertible, we can twist the (scalar multiplication part of the) $\mathbb{k}$-vector space structure of $R\left(\cong R^{(1)}\right.$ as sets) to make $\iota$ into a $\mathbb{k}$-linear map without making it into an $R$-linear map (when $R \neq \mathbb{k}$ ).

Definition 3.2. (Frobenius splitting of a scheme). Let $\mathfrak{X}$ be a separated scheme defined over $\mathbb{k}$. Let $\mathrm{Fr}$ be the (relative) Frobenius endomorphism of $\mathfrak{X}$ (which induces a $\mathbb{k}$-linear endomorphism). We have a natural inclusion $\iota: \mathcal{O}_{\mathfrak{X}} \rightarrow \mathrm{Fr}_{*} \mathcal{O}_{\mathfrak{X}}$. A Frobenius splitting of $\mathfrak{X}$ is a $\mathcal{O}_{\mathfrak{X}}$-linear morphism $\phi: \operatorname{Fr}_{*} \mathcal{O}_{\mathfrak{X}} \rightarrow \mathcal{O}_{\mathfrak{X}}$ such that the composition $\phi \circ \imath$ is the identity.

Definition 3.3. (Compatible splitting). Let $\mathfrak{Y} \subset \mathfrak{X}$ be a closed immersion of separated schemes defined over $\mathbb{k}$. A Frobenius splitting $\phi$ of $\mathfrak{X}$ is said to be compatible with $\mathfrak{Y}$ if $\phi\left(\mathrm{Fr}_{*} \mathcal{I}_{\mathfrak{Y}}\right) \subset \mathcal{I}_{\mathfrak{Y}}$, where $\mathcal{I}_{\mathfrak{Y}}:=\operatorname{ker}\left(\mathcal{O}_{\mathfrak{X}} \rightarrow \mathcal{O}_{\mathfrak{Y}}\right)$. Compatible Frobenius splitting of a pair of a commutative ring and its quotient ring is defined through their spectra.

Remark 3.4. A Frobenius splitting of $\mathfrak{X}$ compatible with $\mathfrak{Y}$ induces a Frobenius splitting of $\mathfrak{Y}$ (see, e.g., [14, Remark 1.1.4 (ii)]).

Theorem 3.5 ([14]). Let $\mathfrak{X}$ be a separated scheme of finite type over $\mathbb{k}$ with semiample line bundles $\mathcal{L}_{1}, \ldots, \mathcal{L}_{r}$. If $\mathfrak{X}$ admits a Frobenius splitting, then the multisection ring

$$
\bigoplus_{n_{1}, \ldots, n_{r} \geq 0} \Gamma\left(\mathfrak{X}, \mathcal{L}_{1}^{\otimes n_{1}} \otimes \cdots \otimes \mathcal{L}_{r}^{\otimes n_{r}}\right)
$$

admits a Frobenius splitting $\phi$. Moreover, a closed subscheme $\mathfrak{Y} \subset \mathfrak{X}=$ Proj $R$ admits a compatible Frobenius splitting if and only if the homogeneous ideal $I_{\mathfrak{Y}} \subset R$ that defines $\mathfrak{Y}$ satisfies $\phi\left(I_{\mathfrak{Y}}\right) \subset I_{\mathfrak{Y}}-$ that is, the pair $\left(R, R / I_{\mathfrak{Y}}\right)$ admits a compatible Frobenius splitting $\phi$. 
Definition 3.6. (Canonical splitting). Let $\mathfrak{X}$ be a separated scheme over $\mathbb{k}$ equipped with a $B$-action. A Frobenius splitting $\phi$ is said to be $B$-canonical if it is $H$-fixed and each $i \in$ I yields

$$
\rho_{\alpha_{i}}\left(z^{p}\right) \phi\left(\rho_{\alpha_{i}}(-z) f\right)=\sum_{j=0}^{p-1} \frac{z^{j}}{j !} \phi_{i, j}(f), \quad z \in \mathbb{k},
$$

where $\phi_{i, j} \in \operatorname{Hom}_{\mathcal{O}_{\mathfrak{X}}}\left(\mathrm{Fr}_{*} \mathcal{O}_{\mathfrak{X}}, \mathcal{O}_{\mathfrak{X}}\right)$. We similarly define the notion of $B^{-}$-canonical splitting (resp., I-canonical splitting and $\mathbf{I}^{-}$-canonical splitting) by using $\left\{\rho_{-\alpha_{i}}\right\}_{i \in \mathrm{I}}$ (resp., $\left\{\rho_{\alpha_{i}}\right\}_{i \in \mathrm{I}_{\mathrm{af}}}$ and $\left\{\rho_{-\alpha_{i}}\right\}_{i \in \mathrm{I}_{\mathrm{af}}}$ ) instead. Canonical splitting of a commutative ring $S$ over $\mathbb{k}$ is defined through its spectrum.

Proposition 3.7. (Compare [14]). Let $S=\bigoplus_{m \geq 0} S_{m}$ be a graded ring with $S_{0}=\mathbb{k}$ such that

- $S$ is equipped with a degree-preserving I-action,

$\circ$ each $S_{m}$ is a graded $\mathbb{k}$-vector space compatible with the multiplication and

$\circ$ we have an I-canonical Frobenius splitting $\phi: S^{(1)} \rightarrow S$.

Then the induced map

$$
\phi^{\vee}: S_{m}^{\vee} \longrightarrow S_{p m}^{\vee}, \quad m \in \mathbb{Z}_{\geq 0}
$$

satisfies

$$
\phi^{\vee}\left(E_{i}^{(n)} \mathbf{v}\right)=E_{i}^{(p n)} \phi^{\vee}(\mathbf{v}) \quad \forall i \in \mathrm{I}_{\mathrm{af}}, n \in \mathbb{Z}_{\geq 0}, \mathbf{v} \in S_{m}^{\vee}
$$

Similar results hold for the $\mathbf{I}^{-}$- and $B^{ \pm}$-actions.

Remark 3.8. In our opinion, one merit of Proposition 3.7 over [14, Proposition 4.1.8] is that it becomes obvious that a projective variety $\mathfrak{X}$ with a $B$-action has at most one $B$-canonical splitting whenever the space of global sections of all ample line bundles is (or can be made) $B$-cocyclic compatible with multiplications (compare [14, Theorem 4.1.15] and Corollary 3.35).

Proof of Proposition 3.7. The condition that $S_{m}$ is a graded vector space implies $S_{m} \stackrel{\cong}{\longrightarrow}\left(S_{m}^{\vee}\right)^{\vee}$ for each $m \in \mathbb{Z}_{\geq 0}$. By [14, Proposition 4.1.8], each $\mathbf{w} \in S_{p m} \subset S^{(1)}$ satisfies $\phi\left(E_{i}^{(p n)} \mathbf{w}\right)=E_{i}^{(n)} \phi(\mathbf{w})$ for $i \in \mathrm{I}_{\text {af }}$ and $n \geq 0$. Using the natural nondegenerate invariant pairing $\langle\bullet, \bullet\rangle$ between $S_{m}^{\vee}$ and $S_{m}$, we compute the leftmost term of

$$
\left\langle\mathbf{v}, \phi\left(E_{i}^{(p)} \mathbf{w}\right)\right\rangle=\left\langle\mathbf{v}, E_{i} \phi(\mathbf{w})\right\rangle=-\left\langle\phi^{\vee}\left(E_{i} \mathbf{v}\right), \mathbf{w}\right\rangle
$$

by using the invariance under the corresponding unipotent action, yielding

$$
\begin{aligned}
\left\langle\mathbf{v}, \phi\left(E_{i}^{(p)} \mathbf{w}\right)\right\rangle & =-\sum_{k_{1}=1}^{p}\left\langle E^{\left(k_{1}\right)} \phi^{\vee}(\mathbf{v}), E_{i}^{\left(p-k_{1}\right)} \mathbf{w}\right\rangle \\
& =\sum_{m=1}^{p} \sum_{k_{\bullet}>0, k_{1}+k_{2}+\cdots+k_{m}=p}(-1)^{m}\left\langle E_{i}^{\left(k_{1}\right)} E_{i}^{\left(k_{2}\right)} \cdots \phi^{\vee}(\mathbf{v}), \mathbf{w}\right\rangle \\
& =-\left\langle E^{(p)} \phi^{\vee}(\mathbf{v}), \mathbf{w}\right\rangle,
\end{aligned}
$$

since we have $E_{i}^{\left(k_{1}\right)} E_{i}^{\left(k_{2}\right)} \cdots E_{i}^{\left(k_{m}\right)} \in p \mathbb{Z} E_{i}^{(p)}$ except for $k_{1}=p, 0=k_{2}=\cdots$. This implies the case when $n=1$. 
Similarly, we have

$$
\left\langle\mathbf{v}, \phi\left(E_{i}^{(p n)} \mathbf{w}\right)\right\rangle=\sum_{m=1}^{n} \sum_{k_{\bullet}>0, k_{1}+k_{2}+\cdots+k_{m}=n}(-1)^{m}\left\langle E_{i}^{\left(p k_{1}\right)} E_{i}^{\left(p k_{2}\right)} \cdots \phi^{\vee}(\mathbf{v}), \mathbf{w}\right\rangle .
$$

Compared with

$$
\left\langle\mathbf{v}, E_{i}^{(n)} \phi(\mathbf{w})\right\rangle=\sum_{m=1}^{n} \sum_{k_{\bullet}>0, k_{1}+k_{2}+\cdots+k_{m}=n}(-1)^{m}\left\langle\phi^{\vee}\left(E_{i}^{\left(k_{1}\right)} E_{i}^{\left(k_{2}\right)} \cdots \mathbf{v}\right), \mathbf{w}\right\rangle
$$

using induction on $n$, we conclude the result.

\subsection{Representations of affine Lie algebras over $\mathbb{Z}$}

In this section, we systematically use global basis theory [41, 43, 44, 45, 65, 35] by specialising the quantum parameter q to 1 . Therefore, we might refer to these references without an explicit declaration that we specialise $q$.

We consider the Kostant-Lusztig $\mathbb{Z}$-form $U_{\mathbb{Z}}^{+}\left(\right.$resp., $\left.U_{\mathbb{Z}}^{-}\right)$of $U([\mathfrak{I}, \mathfrak{I}])\left(\operatorname{resp} ., U\left(\left[\mathfrak{I}^{-}, \mathfrak{I}^{-}\right]\right)\right)$obtained as the specialisation $q=1$ of the $\mathbb{Z}\left[q, q^{-1}\right]$-integral form of the quantised enveloping algebras [66, $\S 23.2]$.

Remark 3.9. We remark that $U_{\mathbb{Z}}^{ \pm}$are the same integral forms dealt with in [31], and also coincide with the integral forms obtained through the Drinfeld presentation $([4, \S 2]$ and [73, Lemma 2.5]).

Note that $U_{\mathbb{Z}}^{ \pm}$are equipped with the $\mathbb{Z}$-bases $\mathbf{B}(\mp \infty)$ obtained by the specialisation $q=1$ of the lower global basis [41] (see also [66, §25]). In view of [65, 43], we have an idempotent $\mathbb{Z}$-integral form

$$
\dot{U}_{\mathbb{Z}}=\bigoplus_{\Lambda \in P^{\mathrm{af}}} U_{\mathbb{Z}}^{-} U_{\mathbb{Z}}^{+} a_{\Lambda}
$$

such that

$$
a_{\Lambda} a_{\Gamma}=\delta_{\Lambda, \Gamma} a_{\Lambda}, \quad \Lambda, \Gamma \in P^{\mathrm{af}},
$$

and

$$
E_{i}^{(m)} a_{\Lambda}=a_{\Lambda+m \alpha_{i}} E_{i}^{(m)}, \quad F_{i}^{(m)} a_{\Lambda}=a_{\Lambda-m \alpha_{i}} F_{i}^{(m)}, \quad i \in \mathrm{I}_{\mathrm{af}}, m \in \mathbb{Z}_{\geq 0} .
$$

We set $\dot{U}_{\mathbb{Z}}^{\geq 0} \subset \dot{U}_{\mathbb{Z}}$ to be the subalgebra generated by $\left\{F_{i}^{(m)}\right\}_{i \in \mathrm{I}, m \in \mathbb{Z}_{\geq 0}},\left\{a_{\Lambda}\right\}_{\Lambda \in P^{\text {af }}}$ and $U_{\mathbb{Z}}^{+}$.

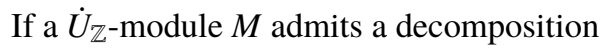

$$
M=\bigoplus_{\Lambda \in P^{\text {af }}} a_{\Lambda} M
$$

then we call this the $P^{\text {af }}$-weight decomposition. If $\Lambda \in P^{\text {af }}$ satisfies $a_{\Lambda} M \neq 0$, then we call $\Lambda$ a $P^{\text {af }}$ weight of $M$. When $M$ is defined over a field $\mathbb{k}$, we define the $P^{\text {af }}$-character of $M$ as

$$
\operatorname{gch} M:=\sum_{\Lambda \in P^{\mathrm{af}}} e^{\Lambda} \operatorname{dim}_{\mathbb{K}} a_{\Lambda} M
$$

whenever the right-hand side makes sense. For each $n \in \mathbb{Z}$, we set

$$
M_{n}:=\sum_{\Lambda \in P^{\mathrm{af}},\langle d, \Lambda\rangle=n} a_{\Lambda} M \subset M
$$


and call it the $d$-degree $n$-part of $M$. Note that these are consistent with formula (2.3) through the identification $q=e^{\delta}$.

For each $\lambda \in P$, we set

$$
a_{\lambda}^{0} M:=\sum_{\Lambda \in P^{\mathrm{af}}, \lambda=\bar{\Lambda}} a_{\Lambda} M .
$$

We call the decomposition

$$
M=\bigoplus_{\lambda \in P} a_{\lambda}^{0} M
$$

the $P$-weight decomposition. We call a nonzero element of $a_{\Lambda} M$ (resp., $a_{\lambda}^{0} M$ ) a $P^{\text {af }}$-weight vector of $M$ (resp., a $P$-weight vector of $M$ ). We also call $\lambda \in P$ with $a_{\lambda}^{0} M \neq\{0\}$ a $P$-weight of $M$.

Similarly, we have the Kostant-Lusztig $\mathbb{Z}$-form $U_{\mathbb{Z}}^{0,+}$ (resp., $U_{\mathbb{Z}}^{0,-}$ ) of $U(\mathfrak{n})$ (resp., $U\left(\mathfrak{n}^{-}\right)$). We have $U_{\mathbb{Z}}^{0,+} \subset U_{\mathbb{Z}}^{+}$and $U_{\mathbb{Z}}^{0,-} \subset U_{\mathbb{Z}}^{-}$. In view of the characterisation of global bases ([41]), we find that $\mathbf{B}^{0}(\mp \infty):=\mathbf{B}(\mp \infty) \cap U_{\mathbb{Z}}^{0, \pm}$ define $\mathbb{Z}$-bases of $U^{0, \pm}$.

We set $\dot{U}_{\mathbb{Z}}^{0} \subset \dot{U}_{\mathbb{Z}}$ to be the subalgebra of $\dot{U}_{\mathbb{Z}}$ generated by $\left\{E_{i}^{(m)}, F_{i}^{(m)}\right\}_{i \in \mathrm{I}, m \in \mathbb{Z}_{\geq 0}},\left\{a_{\Lambda}\right\}_{\Lambda \in P^{\text {af }}}$. For a field $\mathbb{k}$, a $\dot{U}_{\mathbb{k}}^{\geq 0}$-module $M$ with a $P^{\text {af }}$-weight decomposition is said to be $\dot{U}_{\mathbb{k}}^{0}$-integrable if its $\left\{E_{i}^{(m)}, F_{i}^{(m)}\right\}_{m \geq 0}$ action induces an $S L(2, i)_{\mathbb{K}}$-action whose $(S L(2, i) \cap H)_{\mathbb{K}}$-eigenvalues are given by the $P$-weights for each $i \in \mathrm{I}$.

Note that if a $U\left(\widetilde{\mathfrak{g}}_{\mathbb{C}}\right)$-module $V$ over $\mathbb{C}$ carries a cyclic $\widetilde{\mathfrak{h}}_{\mathbb{C}}$-weight vector whose weight belongs to $P^{\text {af }}$ and each of its $\widetilde{\mathfrak{h}}_{\mathbb{C}}$-weight spaces is finite-dimensional, then we have a $\dot{U}_{\mathbb{Z}}$-lattice $V_{\mathbb{Z}}$ inside $V$. In such a case, the module $V_{\mathbb{Z}} \otimes_{\mathbb{Z}} \mathbb{K}$ admits $P^{\text {af }}$ - or $P$-weight decompositions.

The Chevalley involution of $\dot{U}_{\mathbb{Z}}$ is defined as

$$
\theta\left(E_{i}^{(m)}\right)=F_{i}^{(m)}, \theta\left(F_{i}^{(m)}\right)=E_{i}^{(m)} \quad \text { and } \quad \theta\left(a_{\Lambda}\right)=a_{-\Lambda}, \quad i \in \mathrm{I}_{\mathrm{af}}, m \in \mathbb{Z}_{\geq 0}, \Lambda \in P^{\mathrm{af}} .
$$

Definition 3.10 ([45]). A $U\left(\widetilde{\mathfrak{g}}_{\mathbb{C}}\right)$-module $V$ over $\mathbb{C}$ with a cyclic $\widetilde{\mathfrak{h}}_{\mathbb{C}}$-weight vector $\mathbf{v}$ is said to be compatible with the negative global basis if we have

$$
U_{\mathbb{Z}}^{-} \mathbf{v}=\bigoplus_{b \in \mathbf{B}(\infty)} \mathbb{Z} b \mathbf{v} \subset V .
$$

If $(V, \mathbf{v})$ is compatible with the negative global basis, then we set

$$
\mathbf{B}^{-}(V)=\mathbf{B}^{-}(V, \mathbf{v}):=\{b \mathbf{v} \mid b \in \mathbf{B}(\infty) \text { such that } b \mathbf{v} \neq 0\} \subset V
$$

and refer to them as the negative global basis of $V$.

Compatibility with the positive global basis and the positive global basis $\mathbf{B}^{+}(V)=\mathbf{B}^{+}(V, \mathbf{v})$ of $V$ is defined similarly.

Theorem 3.11 ([41]). We have the following:

1. For each $\Lambda \in P_{+}^{\mathrm{af}}$, the $\widetilde{\mathfrak{g}}_{\mathbb{C}}$-module $L(\Lambda)_{\mathbb{C}}$ is compatible with the negative global basis.

2. For each $\lambda \in P_{+}$, we have

$$
V(\lambda)_{\mathbb{C}}=\bigoplus_{b \in \mathbf{B}^{0}(\infty)} \mathbb{C} b \mathbf{v}_{\lambda}^{0} .
$$

We set $\mathbf{B}(\Lambda):=\mathbf{B}^{-}\left(L(\Lambda)_{\mathbb{C}}, \mathbf{v}_{\Lambda}\right)$ for each $\Lambda \in P_{+}^{\text {af }}$. 
For each $\Lambda \in P_{+}^{\text {af }}$ and $\lambda \in P_{+}$, we set

$$
L(\Lambda)_{\mathbb{Z}}:=U_{\mathbb{Z}}^{-} \mathbf{v}_{\Lambda} \subset L(\Lambda)_{\mathbb{C}} \quad \text { and } \quad V(\lambda)_{\mathbb{Z}}:=\left(U_{\mathbb{Z}}^{0,-}\right) \mathbf{v}_{\lambda}^{0} \subset V(\lambda)_{\mathbb{C}}
$$

Here $V(\lambda)_{\mathbb{Z}}$ acquires the action of $\dot{U}_{\mathbb{Z}}^{0}$ thanks to the splitting $P \hookrightarrow P^{\text {af }}$.

Corollary 3.12. We have the following:

1. For each $\Lambda, \Gamma \in P_{+}^{\text {af }}$, we have a natural inclusion $L(\Lambda+\Gamma)_{\mathbb{Z}} \hookrightarrow L(\Lambda)_{\mathbb{Z}} \otimes_{\mathbb{Z}} L(\Gamma)_{\mathbb{Z}}$ of $\dot{U}_{\mathbb{Z}-\text { modules, }}$, which is a direct summand as $\mathbb{Z}$-modules.

2. For each $\lambda, \mu \in P_{+}$, we have a natural inclusion $V(\lambda+\mu)_{\mathbb{Z}} \hookrightarrow V(\lambda)_{\mathbb{Z}} \otimes_{\mathbb{Z}} V(\mu)_{\mathbb{Z}}$ of $\dot{U}_{\mathbb{Z}}^{0}$-modules, which is a direct summand as $\mathbb{Z}$-modules.

Proof. Since the two cases are completely parallel, we prove only the first case. The $\widetilde{\mathfrak{g}}$-module $L(\Lambda)_{\mathbb{C}} \otimes_{\mathbb{C}}$ $L(\Gamma)_{\mathbb{C}}$ decomposes into the direct sum of integrable highest weight modules ([40, Proposition 9.10]), with a direct summand $L(\Lambda+\Gamma)_{\mathbb{C}}$. In view of [41, Theorem 3], it gives rise to the $\mathbb{Z}$ [q]-lattice of the quantised version of $L(\Lambda) \otimes L(\Gamma)$ compatible with those of $L(\Lambda+\Gamma)$ via the natural embedding. By setting $q=1$, we obtain a direct sum decomposition of $L(\Lambda)_{\mathbb{Z}} \otimes_{\mathbb{Z}} L(\Gamma)_{\mathbb{Z}}$ as $\mathbb{Z}$-modules with its direct summand $L(\Lambda+\Gamma)_{\mathbb{Z}}$.

Theorem 3.13 ([43]). For each $\lambda \in P_{+}$, the $\widetilde{\mathfrak{g}}_{\mathbb{C}}$-module $\mathbb{X}(\lambda)_{\mathbb{C}}$ is compatible with the negative/positive global basis (for every extremal weight vector).

For each $\lambda \in P_{+}$, we set

$$
\mathbb{X}(\lambda)_{\mathbb{Z}}:=\dot{U}_{\mathbb{Z}} \mathbf{v}_{\lambda} \subset \mathbb{X}(\lambda)_{\mathbb{C}}
$$

Theorem 3.14 ([45]). Set $\lambda \in P_{+}$. There exists a $\mathbb{Z}$-basis $\mathbf{B}(\mathbb{X}(\lambda))$ of $\mathbb{X}(\lambda)_{\mathbb{Z}}$ that contains the negative/positive global basis of $\mathbb{X}(\lambda)_{\mathbb{Z}}$ constructed from every extremal weight vector of $\mathbb{X}(\lambda)_{\mathbb{C}}$.

Proof. We set $\mathbf{B}(\mathbb{X}(\lambda))$ to be the specialisation of the global basis of a quantum loop algebra module [43, Proposition 8.2.2]. Then it is compatible with the global basis generated from extremal weight vectors by [45, Theorem 3.3].

For each $\lambda \in P_{+}$and $w \in W_{\text {af }}$, we define

$$
\mathbb{W}_{w}(\lambda)_{\mathbb{Z}}:=U_{\mathbb{Z}}^{+} \mathbf{v}_{w \lambda} \subset \mathbb{X}(\lambda)_{\mathbb{C}} \quad \text { and } \quad \mathbb{W}_{w}^{-}(\lambda)_{\mathbb{Z}}:=U_{\mathbb{Z}}^{-} \mathbf{v}_{w \lambda} \subset \mathbb{X}(\lambda)_{\mathbb{C}}
$$

We set $\mathbb{W}(\lambda)_{\mathbb{Z}}:=\mathbb{W}_{w_{0}}(\lambda)_{\mathbb{Z}}$ and $\mathbb{W}^{-}(\lambda)_{\mathbb{Z}}:=\mathbb{W}_{e}^{-}(\lambda)_{\mathbb{Z}}$.

Lemma 3.15 (Naito and Sagaki). For each $\lambda \in P_{+}$and $w, v \in W_{\mathrm{af}}$, we have $\mathbb{W}_{w w_{0}}(\lambda)_{\mathbb{Z}} \subset \mathbb{W}_{v w_{0}}(\lambda)_{\mathbb{Z}}$ if $w \leq_{\frac{\infty}{2}} v$. If we have $\lambda \in P_{++}$in addition, then we have $\mathbb{W}_{w w_{0}}(\lambda)_{\mathbb{Z}} \subset \mathbb{W}_{v w_{0}}(\lambda)_{\mathbb{Z}}$ if and only if $w \leq \frac{\infty}{2} v$.

Proof. Apply the inclusion relation of the (labels of the) global basis in [72, Corollary 5.2.5] (see also $[45, \S 2.8])$.

Corollary 3.16. For each $\lambda \in P_{+}, w \in W_{\mathrm{af}}$ and $i \in \mathrm{I}_{\mathrm{af}}$, we have $\mathbb{W}_{s_{i} w w_{0}}(\lambda)_{\mathbb{K}} \subset \mathbb{W}_{w w_{0}}(\lambda)_{\mathbb{K}}$ if $s_{i} w \leq \frac{\infty}{2} w$. In this case, $\mathbb{W}_{w w_{0}}(\lambda)_{\mathbb{K}}$ inherits an action of $S L(2, i)_{\mathbb{K}}$ from $\mathbb{X}(\lambda)_{\mathbb{K}}$.

Proof. The first part of the assertion is the special case of Lemma 3.15. Given this, it remains to notice that a lift of $s_{i} \in W_{\text {af }}$ sends $\mathbf{v}_{w w_{0} \lambda}$ to $\pm \mathbf{v}_{s_{i} w w_{0} \lambda}$, and hence the Bruhat decomposition of $\mathbf{I}(i)_{\mathbb{K}}$ (into two $\mathbf{I}_{\mathbb{K}}$-double cosets) implies that $\mathbb{W}_{w w_{0}}(\lambda)_{\mathbb{K}}$ is stable under $\mathbf{I}(i)_{\mathbb{K}}$.

Lemma 3.17. For each $\lambda \in P_{+}$and $w \in W_{\mathrm{af}}$, we have the following:

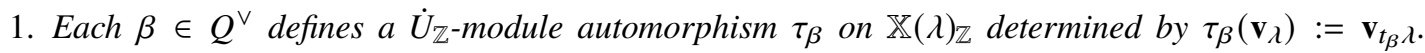
Moreover, $\tau_{\beta} \mathbf{B}(\mathbb{X}(\lambda))=\mathbf{B}(\mathbb{X}(\lambda))$.

2. $\theta^{*}\left(\mathbb{X}(\lambda)_{\mathbb{Z}}\right) \cong \mathbb{X}\left(-w_{0} \lambda\right)_{\mathbb{Z}}$ as $\dot{U}_{\mathbb{Z}}$-modules. Moreover, $\theta^{*} \mathbf{B}(\mathbb{X}(\lambda))=\mathbf{B}\left(\mathbb{X}\left(-w_{0} \lambda\right)\right)$. 
3. $\mathbb{W}_{w}(\lambda)_{\mathbb{Z}}=\mathbb{W}_{w}(\lambda)_{\mathbb{C}} \cap \mathbb{X}(\lambda)_{\mathbb{Z}}$.

4. There is a $U_{\mathbb{Z}}^{-}$-cyclic vector of $\theta^{*}\left(\mathbb{W}_{w}(\lambda)_{\mathbb{Z}}\right)$ with weight $-w \lambda=w_{0}\left(-w_{0} \lambda\right)$. In particular,

$$
\theta^{*}\left(\mathbb{W}_{w}(\lambda)_{\mathbb{Z}}\right) \cong \mathbb{W}_{w w_{0}}^{-}\left(-w_{0} \lambda\right)_{\mathbb{Z}} \quad \text { and } \quad \theta^{*}\left(\mathbb{W}_{w}^{-}(\lambda)_{\mathbb{Z}}\right) \cong \mathbb{W}_{w w_{0}}\left(-w_{0} \lambda\right)_{\mathbb{Z}}
$$

Proof. We borrow the setting of [43, §8.1 and $\S 8.2]$.

We prove the first assertion. Since $\mathbf{v}_{\lambda}$ and $\mathbf{v}_{t_{\beta} \lambda}$ obey the same relation, $\tau_{\beta}$ defines an automorphism as $\widetilde{\mathfrak{g}}_{\mathbb{C}}$-modules. The latter assertion follows from Theorem 3.14.

We prove the second assertion. The defining equation of $\theta^{*}\left(\mathbf{v}_{\lambda}\right)$ is the same as the cyclic vector $\mathbf{v}_{-w_{0} \lambda} \in$ $\mathbb{X}\left(-w_{0} \lambda\right)_{\mathbb{C}}$ as $\widetilde{\mathfrak{g}}_{\mathbb{C}}$-modules. This yields a $\widetilde{\mathfrak{g}}_{\mathbb{C}}$-module isomorphism $\eta: \theta^{*}\left(\mathbb{X}(\lambda)_{\mathbb{C}}\right) \longrightarrow \mathbb{X}\left(-w_{0} \lambda\right)_{\mathbb{C}}$. Since $\theta$ exchanges $U_{\mathbb{Z}}^{ \pm}$and $\mathbf{v}_{\lambda}$ is cyclic, we deduce that $\eta\left(\theta^{*}\left(\mathbb{X}(\lambda)_{\mathbb{Z}}\right)\right)=\dot{U}_{\mathbb{Z}} \mathbf{v}_{-\lambda} \subset \mathbb{X}\left(-w_{0} \lambda\right)_{\mathbb{C}}$. By Theorem 3.14, we conclude $\theta^{*} \mathbf{B}(\mathbb{X}(\lambda))=\mathbf{B}\left(\mathbb{X}\left(-w_{0} \lambda\right)\right)$, as required.

We prove the third assertion. By Theorem 3.14, the $\mathbb{Z}$-basis of $\mathbb{W}(\lambda)_{\mathbb{Z}}$ is formed by the nonzero elements of $\mathbf{B}(-\infty) \mathbf{v}_{w_{0} \lambda}$ and forms a direct summand of $\mathbb{X}(\lambda)_{\mathbb{Z}}$ as $\mathbb{Z}$-modules. Hence, the case where $w=w_{0}$ follows. For $w \in W$, we apply [43, Lemma 8.2.1] repeatedly to deduce the assertion from the $w=w_{0}$ case by using $\mathbf{B}(-\infty) \mathbf{v}_{w \lambda} \subset \mathbf{B}(-\infty) \mathbf{v}_{w_{0} \lambda}$. For $w=u t_{\beta} \in W_{\text {af }}$ with $u \in W, \beta \in Q^{\vee}$, we additionally apply $\tau_{w_{0} \beta}$ to conclude the assertion.

We prove the fourth assertion. The vector $\theta^{*}\left(\mathbf{v}_{w \lambda}\right)$ is a $U_{\mathbb{Z}}^{-}$-cyclic vector of $\theta^{*}\left(\mathbb{W}_{w}(\lambda)_{\mathbb{Z}}\right)$, and its weight is

$$
-w \lambda=w w_{0}\left(-w_{0} \lambda\right)
$$

Hence, we conclude the assertion (using the fact that $\theta$ is an involution).

Theorem 3.18 ([44] and [5]). Set $\lambda \in P_{+}$. The unique (d-degree 0$) \dot{U}_{\mathbb{Z}}^{\geq 0}$-module map

$$
\Psi_{\lambda}: \mathbb{W}(\lambda)_{\mathbb{Z}} \hookrightarrow \bigotimes_{i \in \mathrm{I}} \mathbb{W}\left(\varpi_{i}\right)_{\mathbb{Z}}^{\otimes\left\langle\alpha_{i}^{\vee}, \lambda\right\rangle},
$$

which sends $\mathbf{v}_{\lambda}$ to the tensor product of $\left\{\mathbf{v}_{\varpi_{i}}\right\}_{i \in \mathrm{I}} s$, is injective and defines a direct summand as $\mathbb{Z}$ modules.

Proof. We set $\mathbb{X}^{\otimes}:=\bigotimes_{i \in \mathrm{I}} \mathbb{X}\left(\varpi_{i}\right)_{\mathbb{Z}}^{\otimes\left\langle\alpha_{i}^{\vee}, \lambda\right\rangle}$. The map $\Psi_{\lambda}$ exists, as $\bigotimes_{i \in \mathrm{I}} \mathbf{v}_{\varpi_{i}}^{\otimes\left\langle\alpha_{i}^{\vee}, \lambda\right\rangle}$ obeys the same defining condition as the extremal weight vector $\mathbf{v}_{\lambda} \in \mathbb{X}(\lambda)_{\mathbb{Z}}$, and the comultiplication of $\widetilde{\mathfrak{g}}$ induces an algebra map $\triangle: \dot{U}_{\mathbb{Z}} \subset \dot{U}_{\mathbb{Z}} \otimes \dot{U}_{\mathbb{Z}}([66, \S 23.1 .5])$.

The map $\Psi_{\lambda}$ is injective by [5, Corollary 4.15 and Remark 4.17]. By [44, Theorem 8.5], the $q=1$ specialisations of the global bases yield the $\dot{U}_{\mathbb{Z}}$-spans of the extremal weight vectors in $\mathbb{X}(\lambda)_{\mathbb{Z}}$ and $\mathbb{X}^{\otimes}$ up to the action of some rings of (partially symmetric) polynomials with integer coefficients, respectively. This yields a splitting of $\Psi_{\lambda}$ as $\mathbb{Z}$-modules, where the $\mathbb{Z}$-module structure of the right-hand side comes from the crystal lattice of (the q-version of) $\mathbb{X}^{\otimes}$ (as in [44, (8.8)]). We call this crystal lattice $L_{1}$.

We have another $\mathbb{Z}[q]$-lattice inside (the q-version of) $\mathbb{X}^{\otimes}$ obtained by the tensor product of the crystal lattices of (the q-versions of) $\mathbb{X}\left(\varpi_{i}\right) \mathrm{s}$. We call this $\mathbb{Z}[q]$-lattice $\mathrm{L}_{2}$.

By [44, Proposition 8.6], the global basis of $L_{1}$ is written in terms of the (tensor product) global bases of $L_{2}$ by an upper-unitriangular matrix $C$ (valued in $\mathbb{Q}[q]$, with finitely many nonzero entries in each row). Hence, the corresponding bases in $\mathbb{X}^{\otimes}$ are related by an upper-unitriangular matrix $C$ obtained as the $q=1$ specialisation of $C$ (valued in $\mathbb{Q}$ ). In view of the fact that $\Delta$ is an algebra morphism (and [44, Theorem 8.5]), we deduce $L_{1} \subset L_{2}$. Hence, we have $\mathbb{Z} \otimes_{\mathbb{Z}[q]} L_{1} \subset \mathbb{Z} \otimes_{\mathbb{Z}[q]} L_{2} \subset \mathbb{X}^{\otimes}$. In particular, the matrix $C$ must be valued in $\mathbb{Z}$. This implies $\mathbb{Z} \otimes_{\mathbb{Z}[q]} L_{1}=\mathbb{Z} \otimes_{\mathbb{Z}[q]} L_{2}$.

Hence, the $\mathbb{Z}$-module splitting in the second paragraph is indeed what we wanted. 


\subsection{Projectivity of the module $\mathbb{W}(\lambda)_{\mathbb{K}}$}

Let $\mathbb{k}$ be a field. We equip $\stackrel{\cup}{\mathbb{k}}_{\mathbb{k}}^{+}:=\bigoplus_{\lambda \in P} U_{\mathbb{k}}^{+} a_{\lambda}^{0}$ with the structure of an algebra by setting $a_{\lambda}^{0} a_{\mu}^{0}=$ $\delta_{\lambda, \mu} a_{\lambda}^{0}(\lambda, \mu \in P)$

$$
E_{0}^{(m)} a_{\lambda}^{0}=a_{\lambda-m \vartheta}^{0} E_{0}^{(m)}, \quad E_{i}^{(m)} a_{\lambda}^{0}=a_{\lambda+m \alpha_{i}}^{0} E_{i}^{(m)} \quad\left(i \in \mathrm{I}, m \in \mathbb{Z}_{\geq 0}, \lambda \in P\right),
$$

and require

$$
\left(\xi a_{\lambda}^{0}\right)\left(\xi^{\prime} a_{\mu}^{0}\right)=\left(\xi \xi^{\prime}\right) a_{\mu}^{0} \quad \text { when } \quad a_{\lambda}^{0} \xi^{\prime} a_{\mu}^{0}=\xi^{\prime} a_{\mu}^{0} \quad\left(\lambda, \mu \in P, \xi, \xi^{\prime} \in U_{\mathbb{k}}^{+}\right) .
$$

Similarly, we define

$$
\stackrel{\circ}{U}_{\mathbb{k}}^{\geq 0}:=\bigoplus_{\lambda \in P} U_{\mathbb{k}}^{0,-} U_{\mathbb{k}}^{+} a_{\lambda}^{0} \quad \text { and } \quad \stackrel{U}{\mathbb{k}}_{\mathbb{k}}^{0}:=\bigoplus_{\lambda \in P} U_{\mathbb{k}}^{0,-} U_{\mathbb{k}}^{0,+} a_{\lambda}^{0}
$$

and regard them as subalgebras of the completions of $\dot{U}_{\mathbb{k}}^{\geq 0}$ and $\dot{U}_{\mathbb{k}}^{0}$ with respect to the idempotents by setting

$$
\sum_{\lambda=\bar{\Lambda}} a_{\Lambda}=a_{\lambda}^{0} \quad\left(\lambda \in P \subset P^{\mathrm{af}}\right) .
$$

The eigendecomposition with respect to $d \in \widetilde{\mathfrak{h}}$ now becomes an external grading of algebras $\dot{U}_{\mathbb{k}}^{+}$and $\stackrel{\circ}{U}_{\mathbb{k}}^{\geq 0}$. We have algebra inclusions

$$
\stackrel{\circ}{U}_{\mathbb{k}}^{+} \subset \stackrel{\circ}{U_{\mathbb{k}}^{\geq 0} \supset \stackrel{\circ}{U}_{\mathbb{k}}^{0}}
$$

Note that we have a surjective algebra map $\dot{U}_{\mathbb{Z}}^{\geq 0} \rightarrow \dot{U}_{\mathbb{Z}}^{0}$, since $a_{\Lambda} \dot{U}_{\mathbb{Z}}^{\geq 0} a_{\Gamma} \neq 0$ implies $\langle d, \Lambda\rangle \geq\langle d, \Gamma\rangle$ and $a_{\Lambda} \dot{U}_{\mathbb{Z}}^{0} a_{\Gamma} \neq 0$ implies $\langle d, \Lambda\rangle=\langle d, \Gamma\rangle$ for $\Lambda, \Gamma \in P^{\text {af }}$. This induces a surjective algebra map $\dot{U}_{\mathbb{Z}}^{\geq 0} \rightarrow \dot{U}_{\mathbb{Z}}^{0}$, which specialises to $\stackrel{\circ}{\mathbb{k}}_{\mathbb{k}}^{\geq 0} \rightarrow \stackrel{\circ}{U}_{\mathbb{k}}^{0}$.

We sometimes regard $\mathbb{W}(\lambda)_{\mathbb{K}}\left(\lambda \in P_{+}\right)$as a $\mathbb{Z}$-graded $\stackrel{\circ}{\mathbb{k}}_{\mathbb{k}}^{+}$-module or a $\mathbb{Z}$-graded $\stackrel{\circ}{U}_{\mathbb{k}}^{\geq 0}$-module by means of (3.2), whose gradings are given by the $d$-degrees.

Proposition 3.19. Let $\mathbb{k}$ be a field and set $\lambda \in P_{+}$. The module $\mathbb{W}(\lambda)_{\mathbb{K}}$ is the projective cover of $V(\lambda)_{\mathbb{K}}$ in the category of $\dot{U}_{\mathbb{k}}^{\geq 0}$-modules that are $\dot{U}_{\mathbb{k}}^{0}$-integrable and whose $P$-weights are contained in Conv $W \lambda \subset P \otimes_{\mathbb{Z}} \mathbb{R}$, where Conv denotes the $\mathbb{R}$-convex hull.

Lemma 3.20. For each $\lambda \in P_{+}$, the module $\mathbb{W}(\lambda)_{\mathbb{K}}$ is free over a polynomial ring and we have a finitedimensional quotient $W(\lambda)_{\mathbb{K}}$ with $\operatorname{dim}_{\mathbb{K}} a_{\lambda}^{0} W(\lambda)_{\mathbb{K}}=1$.

Remark 3.21. The modules $\mathbb{W}(\lambda)_{\mathbb{K}}$ and $W(\lambda)_{\mathbb{K}}\left(\lambda \in P_{+}\right)$are analogues of global and local Weyl modules in $[19,62,18]$ (compare Theorem 2.2). By construction, their characters are the same as those defined over $\mathbb{C}$ (see $[46, \S 1]$ for more discussion).

Proof of Lemma 3.20. Note that the endomorphism $\tau_{\beta}$ in Lemma 3.17 induces an endomorphism of $\mathbf{B}\left(\mathbb{X}\left(\varpi_{i}\right)\right)$ for each $\beta \in Q$ and $i \in \mathrm{I}$, and hence we know the existence of the injective endomorphisms of $\mathbb{W}(\lambda)_{\mathbb{K}}$ that form a polynomial algebra whose graded dimension is equal to

$$
\operatorname{gdim}_{\operatorname{End}} \operatorname{gr}_{\mathfrak{c}]_{\mathbb{C}}} \mathbb{W}(\lambda)_{\mathbb{C}}=\prod_{i \in \mathrm{I}} \prod_{j=1}^{\left\langle\alpha_{i}^{\vee}, \lambda\right\rangle} \frac{1}{1-q^{j}},
$$

which can be read off from [5, Proposition 4.13] (or [46, Theorem 1.4]), through Theorem 3.18. By $[5,18,46]$, it exhausts the $P$-weight $\lambda$-part of $\mathbb{W}(\lambda)_{\mathbb{K}}$, which generates $\mathbb{W}(\lambda)_{\mathbb{K}}$ as a $\stackrel{U}{\mathbb{k}}_{\mathbb{k}}^{\geq 0}$-module. Therefore, we conclude the result. 
The rest of this subsection is devoted to the proof of Proposition 3.19.

We consider the Demazure functor $\mathscr{D}_{w}$ for $w \in W_{\text {af }}$ with respect to $\stackrel{\circ}{U}_{\mathbb{k}}^{+}$(compare [39, 46, 20]). In view of $[45, \S 2.8]$, the character part of the calculation in [46, Theorem 4.13] carries over to our setting, and hence we have

$$
\mathbb{L} \cdot \mathscr{D}_{t_{\beta}}\left(\mathbb{W}(\lambda)_{\mathbb{K}}\right)=\mathscr{D}_{t_{\beta}}\left(\mathbb{W}(\lambda)_{\mathbb{K}}\right) \cong \mathbb{W}(\lambda)_{\mathbb{K}} \otimes_{\mathbb{K}} \mathbb{K}_{-\left\langle\beta, w_{0} \lambda\right\rangle \delta}, \quad \beta \in Q_{<}^{\vee} .
$$

From this, we also derive

$$
\mathbb{L} \cdot \mathscr{D}_{t_{\beta}}\left(W(\lambda)_{\mathbb{K}}\right)=\mathscr{D}_{t_{\beta}}\left(W(\lambda)_{\mathbb{K}}\right) \cong W(\lambda)_{\mathbb{K}} \otimes_{\mathbb{K}} \mathbb{k}_{-\left\langle\beta, w_{0} \lambda\right\rangle \delta}, \quad \beta \in Q_{<}^{\vee},
$$

using the Koszul resolution as in [20, §5.1.4] by Lemma 3.20.

The $d$-gradings of $\varrho_{\mathbb{K}}^{+}, \mathbb{W}(\lambda)$ and $W(\mu)_{\mathbb{K}}$ are concentrated in nonnegative degrees. It follows that the $d$-grading of $\operatorname{Ext}_{U_{\mathbb{k}}^{+}}^{\bullet}\left(\mathbb{W}(\lambda), W(\mu)_{\mathbb{K}}^{*}\right)$ is bounded from above. Moreover, we have

$$
\operatorname{Ext}_{U_{U_{\mathbb{k}}^{+}}^{+}}^{\bullet}\left(\mathscr{D}_{t_{\beta} w_{0}}\left(\mathbb{W}(\lambda)_{\mathbb{K}}\right), W(\mu)_{\mathbb{k}}^{*}\right) \cong \operatorname{Ext}_{U_{U_{\mathbb{k}}^{+}}^{+}}^{\bullet}\left(\mathbb{W}(\lambda)_{\mathbb{K}}, \mathscr{D}_{t_{-w_{0} \beta} w_{0}}\left(W(\mu)_{\mathbb{K}}\right)^{*}\right)
$$

for every $\lambda, \mu \in P_{+}$and $\beta \in Q_{<}^{\vee}$ by repeated application of [25, Proposition 5.7] (the argument of which carries over to our setting). By varying $\beta$, we conclude that

$$
\operatorname{Ext}_{U_{\mathbb{1}}^{+}}^{\bullet}\left(\mathbb{W}(\lambda)_{\mathbb{k}}, W(\mu)_{\mathbb{K}}^{*}\right) \equiv\{0\}, \quad \lambda \neq-w_{0} \mu,
$$

since $-\left\langle\beta, w_{0} \lambda\right\rangle \neq-\left\langle-w_{0} \beta, w_{0} \mu\right\rangle=\langle\beta, \mu\rangle$ for some choice of $\beta$.

Consider the simple integrable $U_{\mathbb{k}}^{0}$-module quotient $L(\lambda)_{\mathbb{K}}$ of $V(\lambda)_{\mathbb{K}}$ for each $\lambda \in P_{+}$.

Lemma 3.22. The set $\left\{L(\lambda)_{\mathbb{K}}\right\}_{\lambda \in P_{+}}$is the complete collection of the isomorphism classes of the irreducible d-graded $\stackrel{\circ}{\mathbb{k}}_{\mathbb{k}}^{0}$-integrable $\stackrel{\circ}{\mathbb{k}}_{\mathbb{k}}^{\geq 0}$-modules (up to d-grading shifts).

Proof. Since $V(\lambda)_{\mathbb{K}}$ surjects onto every irreducible $\stackrel{\circ}{\mathbb{k}}_{\mathbb{k}}^{0}$-module with highest weight $\lambda([1, \S 1.20])$, it follows that it surjects onto every irreducible $d$-graded $U_{\mathbb{k}}^{\geq 0}$-module with highest weight $\lambda$. Since the $P$-weight $\lambda$-part of $V(\lambda)_{\mathbb{K}}$ is one-dimensional, we conclude the result.

We return to the proof of Proposition 3.19. We have

$$
\operatorname{gch} W(\lambda)_{\mathbb{K}} \equiv \operatorname{gch} V(\lambda)_{\mathbb{K}} \equiv \operatorname{gch} L(\lambda)_{\mathbb{K}} \quad \bmod \sum_{\lambda>\mu \in P_{+}} \mathbb{Z}[q] \operatorname{gch} L(\mu)_{\mathbb{K}}
$$

by [62] (compare [18]). Therefore, $W(\lambda)_{\mathbb{K}}$ admits a ( $d$-graded) Jordan-Hölder series as a $\stackrel{\cup}{\mathbb{k}}_{\mathbb{k}}^{\geq 0}$-module whose irreducible constituents are of the form $\left\{L(\mu)_{\mathbb{K}}\right\}_{\mu \leq \lambda}$ (up to $d$-grading shifts). It follows that

$$
\operatorname{Ext}_{U_{\mathbb{E}}^{+}}^{\bullet}\left(\mathbb{W}(\lambda)_{\mathbb{K}}, V(\mu)_{\mathbb{K}}\right)=\operatorname{Ext}_{U_{\mathbb{k}}^{+}}^{\bullet}\left(\mathbb{W}(\lambda)_{\mathbb{K}}, L(\mu)_{\mathbb{K}}\right) \equiv\{0\}, \quad \lambda>\mu \in P_{+},
$$

by a repeated application of the short exact sequences to formula (3.4). Since both $\mathbb{W}(\lambda)_{\mathbb{K}}$ and $L(\lambda)_{\mathbb{K}}$ are $\dot{U}_{\mathbb{k}}^{0}$-integrable, we find

$$
\operatorname{Ext}_{U_{\mathbb{k}} \geq 0}^{1}\left(\mathbb{W}(\lambda)_{\mathbb{L}}, L(\mu)_{\mathbb{L}}\right) \equiv\{0\}, \quad \lambda>\mu \in P_{+} .
$$

From these, it suffices to prove

$$
\operatorname{Ext}_{U_{\mathbb{K}}^{\geq 0}}^{1}\left(\mathbb{W}(\lambda)_{\mathbb{K}}, L(\lambda)_{\mathbb{K}}\right) \equiv\{0\}, \quad \lambda \in P_{+},
$$

to deduce the assertion. 
In view of the fact that $\lambda+\alpha_{i}(i \in \mathrm{I})$ is not a $P$-weight of $\mathbb{W}(\lambda)_{\mathbb{k}}$, the Drinfeld presentation of $\dot{U}_{\mathbb{k}}^{\geq 0}$ ([5, §3]) forces the space of $U_{\mathbb{k}}^{\geq 0}$-module endomorphisms of $\mathbb{W}(\lambda)_{\mathbb{K}}$ to be generated by the images of the imaginary weight vectors $\tilde{P}_{i, m \delta}$ for $i \in \mathrm{I}$ and $m>0$ (defined in [5, (3.7)]). In view of its descriptions ([5, Proposition 3.17] or [17, Lemma 4.5]), we find that $\tilde{P}_{i, m}$ acts on $\mathbf{v}_{\lambda} \in \mathbb{W}(\lambda)_{\mathbb{K}}$ by zero if $\left\langle\alpha_{i}^{\vee}, \lambda\right\rangle>m$. From this, we derive that the $P$-weight $\lambda$-part of $\mathbb{W}(\lambda)_{\mathbb{K}}$ is maximal possible as a cyclic module with a cyclic vector of $P$-weight $\lambda$ in the category of $\stackrel{U}{\mathbb{k}}_{\mathbb{k}}^{\geq 0}$-modules that are $\stackrel{\cup}{\mathrm{K}}_{\mathbb{k}}^{0}$-integrable and whose $P$ -

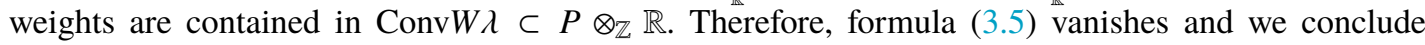
Proposition 3.19.

\subsection{Frobenius splitting of $\mathrm{Q}_{G, \mathrm{~J}}$}

Theorem 3.23. For each $\Lambda \in P_{+}^{\text {af }}$, we have a surjective map $L(\Lambda)_{\mathbb{C}} \rightarrow \mathbb{W}^{-}(\bar{\Lambda})_{\mathbb{C}}$ of $\mathfrak{g}\left[z^{-1}\right]_{\mathbb{C}}$-modules. In addition, this map yields a surjection $L(\Lambda)_{\mathbb{Z}} \rightarrow \mathbb{W}^{-}(\bar{\Lambda})_{\mathbb{Z}}$ of $U_{\mathbb{Z}}^{-}$-modules.

Proof. By [50, Theorem A], the graded $\mathfrak{g}[z]_{\mathbb{C}}$-module $\theta^{*}\left(L(\Lambda)_{\mathbb{C}}\right)$ admits a filtration by the grading shifts of $\left\{\mathbb{W}(\mu)_{\mathbb{C}}\right\}_{\mu \in P_{+}}$. Since the $\left(d\right.$-)degrees of $L(\Lambda)_{\mathbb{C}}$ are concentrated in $\mathbb{Z}_{\leq 0}$ and the degree 0 part of $L(\Lambda)_{\mathbb{C}}$ is $V(\bar{\Lambda})_{\mathbb{C}}$, the first quotient of $\theta^{*}\left(L(\Lambda)_{\mathbb{C}}\right)$ in our filtration must be $\theta^{*}\left(\mathbb{W}^{-}(\bar{\Lambda})_{\mathbb{C}}\right)$. Hence we obtain the surjection $\eta: L(\Lambda)_{\mathbb{C}} \rightarrow \mathbb{W}^{-}(\bar{\Lambda})_{\mathbb{C}}$ of $\mathfrak{g}\left[z^{-1}\right]_{\mathbb{C}}$-modules.

Since both modules share the $U_{\mathbb{C}}^{-}$-cyclic vector and are compatible with the negative global basis, we conclude that the $\mathbb{Z}$-basis of $\mathbb{W}^{-}(\bar{\Lambda})_{\mathbb{Z}}$ is obtained as a $\mathbb{Z}$-basis of $L(\Lambda)_{\mathbb{Z}}$ that is not annihilated by $\eta$. Hence we conclude that $\eta$ induces a surjection $L(\Lambda)_{\mathbb{Z}} \rightarrow \mathbb{W}^{-}(\bar{\Lambda})_{\mathbb{Z}}$ of $U_{\mathbb{Z}}^{-}$-modules.

Corollary 3.24. Let $\mathbb{k}$ be a field. For $\Lambda \in P_{+}^{\text {af }}$, we have a $U_{\mathbb{k}}^{-}$-module generator set $\left\{\mathbf{u}_{m}\right\}_{m \in \mathbb{Z}_{\geq 0}}$ of $\operatorname{ker}\left(L(\Lambda)_{\mathbb{K}} \rightarrow \mathbb{W}^{-}(\bar{\Lambda})_{\mathbb{K}}\right)$ that satisfies the following:

- Each element $\mathbf{u}_{m}$ satisfies $a_{\Lambda_{m}} \mathbf{u}_{m}=\mathbf{u}_{m}$ for some $\Lambda_{m} \in P^{\mathrm{af}}$.

○ For each $m \in \mathbb{Z}_{\geq 0}$, we have $\bar{\Lambda}_{m} \notin \operatorname{ConvW} \bar{\Lambda} \subset P \otimes_{\mathbb{Z}} \mathbb{R}$.

Proof. Note that $L(\Lambda)_{\mathbb{K}}$ has at most countable rank over $\mathbb{k}$, which implies that the generator set is at most countable. As both $L(\Lambda)_{\mathbb{K}}$ and $\mathbb{W}^{-}(\bar{\Lambda})_{\mathbb{K}}$ admit $P^{\text {af }}$-weight decompositions, we deduce the first assertion. Since both modules are $\dot{U}_{\mathbb{k}}^{0}$-integrable and $U_{\mathbb{k}}^{-}$-cyclic, the second assertion follows by Proposition 3.19.

Proposition 3.25. For each $\Lambda, \Gamma \in P_{+}^{\text {af }}$, we have the following commutative diagram of $U_{\mathbb{Z}}^{-}$-modules:

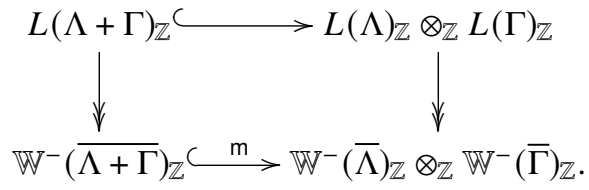

Moreover, all the maps define direct summands as $\mathbb{Z}$-modules.

Proof. The injectivity of the top horizontal arrow and the fact that it defines a direct summand as $\mathbb{Z}$-modules are Corollary 3.12.

The surjectivity of the vertical arrows is Theorem 3.23. Since they are obtained by annihilating parts of $\mathbb{Z}$-bases, these maps define direct summands as $\mathbb{Z}$-modules.

Since all the modules are generated by the cyclic vectors $\mathbf{v}_{\Lambda+\Gamma}$ or $\mathbf{v}_{\Lambda} \otimes \mathbf{v}_{\Gamma}$ as $\mathfrak{g}\left[z^{-1}\right]_{\mathbb{C}}$-modules or $\mathfrak{g}\left[z^{-1}\right]_{\mathbb{C}}^{\oplus 2}$-modules, Theorem 2.3 (twisted by $\theta$ ) implies the injectivity of $\mathrm{m}$ after extending the scalar to C. Hence we deduce

$$
\mathrm{m}\left(\mathbb{W}^{-}(\overline{\Lambda+\Gamma})_{\mathbb{Z}}\right) \subset \mathbb{W}^{-}(\bar{\Lambda})_{\mathbb{Z}} \otimes_{\mathbb{Z}} \mathbb{W}^{-}(\bar{\Gamma})_{\mathbb{Z}}
$$


Therefore, to complete the proof it suffices to prove that formula (3.6) has torsion-free cokernel (as a $\mathbb{Z}$-module). By a repeated use of formula (3.6), we arrive the setting of Theorem 3.18 in view of Theorem 2.3. Thus, the map $m$ defines a direct summand of $\mathbb{W}(\bar{\Lambda})_{\mathbb{Z}} \otimes_{\mathbb{Z}} \mathbb{W}(\bar{\Gamma})_{\mathbb{Z}}$ as $\mathbb{Z}$-modules.

Corollary 3.26. For each $\lambda, \mu \in P_{+}$and $w \in W$, we have the following commutative diagram of $U_{\mathbb{Z}^{+}}^{+}$ modules:

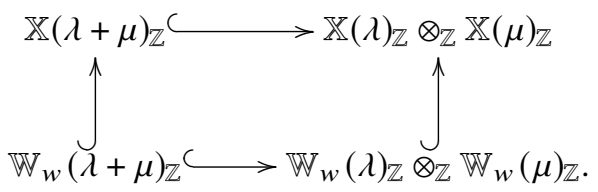

The vertical inclusions are compatible with positive global basis, and the horizontal inclusions define direct summands as $\mathbb{Z}$-modules. In addition, all the inclusions commute with the automorphism $\tau_{\beta}\left(\beta \in Q^{\vee}\right)$ of $\mathbb{X}(\lambda)_{\mathbb{Z}}, \mathbb{X}(\mu)_{\mathbb{Z}}$ and $\mathbb{X}(\lambda+\mu)_{\mathbb{Z}}$.

Proof. In view of Proposition 3.25, the $w=w_{0}$ case follows from Lemma 3.17(1). Thanks to Theorem 2.3 and Lemma 3.17(3), we deduce the general case from the $w=w_{0}$ case.

Set $w \in W_{\text {af }}$ and $\mathrm{J} \subset \mathrm{I}$. We define $P_{+^{-}}$and $P_{\mathrm{J},+^{-}}$graded $\mathbb{Z}$-modules

$$
R^{\mathrm{af}}:=\bigoplus_{\Lambda \in P_{+}^{\mathrm{af}}} L(\Lambda)_{\mathbb{Z}}^{\vee} \quad \text { and } \quad R_{w}(\mathrm{~J}):=\bigoplus_{\lambda \in P_{\mathrm{J},+}} \mathbb{W}_{w w_{0}}(\lambda)_{\mathbb{Z}}^{\vee}
$$

Lemma 3.27. The $\mathbb{Z}$-duals of the horizontal maps in Proposition 3.25 equip $R^{\text {af }}$ and $R_{w}(\mathrm{~J})$ structures of $\left(P_{+^{-}}\right.$and $\left.P_{\mathrm{J}_{+}-}\right)$graded commutative rings.

Proof. The maps in Proposition 3.25 are characterised as the $d$-degree 0 maps of cyclic $U_{\mathbb{Z}}^{-}$-modules, which are unique up to a scalar. Therefore, the composition

$$
\mathbb{W}(\lambda+\mu+\gamma)_{\mathbb{Z}} \hookrightarrow \mathbb{W}(\lambda+\mu)_{\mathbb{Z}} \otimes_{\mathbb{Z}} \mathbb{W}(\gamma)_{\mathbb{Z}} \hookrightarrow \mathbb{W}(\lambda)_{\mathbb{Z}} \otimes_{\mathbb{Z}} \mathbb{W}(\mu)_{\mathbb{Z}} \otimes_{\mathbb{Z}} \mathbb{W}(\gamma)_{\mathbb{Z}}
$$

is the same map as

$$
\mathbb{W}(\lambda+\mu+\gamma)_{\mathbb{Z}} \hookrightarrow \mathbb{W}(\lambda)_{\mathbb{Z}} \otimes_{\mathbb{Z}} \mathbb{W}(\mu+\gamma)_{\mathbb{Z}} \hookrightarrow \mathbb{W}(\lambda)_{\mathbb{Z}} \otimes_{\mathbb{Z}} \mathbb{W}(\mu)_{\mathbb{Z}} \otimes_{\mathbb{Z}} \mathbb{W}(\gamma)_{\mathbb{Z}}
$$

for every $\lambda, \mu, \gamma \in P_{+}$, as the images of the cyclic vectors are the same. Taking their restricted duals implies the associativity of the multiplication of $R_{e}(\mathrm{~J})$. In view of Lemma 3.17(1) and Corollary 3.26, we deduce the associativity of the multiplication of $R_{w}(\mathrm{~J})$ for each $w \in W_{\text {af }}$. The associativity of $R^{\text {af }}$ is proved similarly (compare [49]). The commutativity of $R^{\text {af }}$ and $R_{w}$ ( $\mathrm{J}$ ) follow, as the $\mathrm{q}=1$ coproduct of $\dot{U}_{\mathbb{Z}}$ is symmetric ([66, Lemma $\left.\left.3.1 .4, \S 23.1 .5\right]\right)$.

Corollary 3.28 (Corollary of the proof of Lemma 3.27). For each $w \in W_{\mathrm{af}}$ and $\beta \in Q^{\vee}$, we have an isomorphism of $\left(R_{w}\right)_{\mathbb{Z}}$ and $\left(R_{w t_{\beta}}\right)_{\mathbb{Z}}$ as graded commutative rings equipped with $U_{\mathbb{Z}}^{+}$-actions up to grading twists (given by Lemma 3.17).

We set $R:=R_{e}$. Note that $R_{w}(\mathrm{~J}) \subset R_{w}$ is a subring. We also define

$$
R^{+}(\mathrm{J}):=\bigoplus_{\lambda \in P_{\mathrm{J},+}} \operatorname{Span}_{\mathbb{Z}} \prod_{i \in \mathrm{I}}\left(\mathbb{X}\left(\varpi_{i}\right)_{\mathbb{Z}}^{\vee}\right)^{\left\langle\alpha_{i}^{\vee}, \lambda\right\rangle} \subset \bigoplus_{\lambda \in P_{+}} \mathbb{X}(\lambda)_{\mathbb{Z}}^{\vee}=: \widetilde{R},
$$

where the multiplication is defined through the projective limit formed by the duals of Corollary 3.26. Here we warn that the $\left(\mathbb{Z}\right.$-)rank of some $P^{\text {af }}$-weight space of $\mathbb{X}(\lambda)_{\mathbb{Z}}$ can be infinity, and hence the inclusion $R^{+} \subsetneq \widetilde{R}$ has a huge cokernel. Note that the rank of the $P^{\text {af }}$-weight spaces of $\mathbb{X}\left(\varpi_{i}\right)_{\mathbb{Z}}$ is 
bounded for each $i \in \mathrm{I}$ ([44, Proposition 5.16]), and hence $R^{+}(\mathrm{J})$ has only countably many generators of $P_{\mathrm{J},+}$-degrees $\left\{\varpi_{i}\right\}_{i \in \mathrm{I} \backslash \mathrm{J}}$.

By construction, the rings $R^{\text {af }}, R_{w}(\mathrm{~J})$ and $R^{+}(\mathrm{J})$ are free over $\mathbb{Z}$.

For each $\lambda \in P_{+}$and $w \in W_{\text {af }}$, we have a unique $P^{\text {af }}$-weight vector

$$
\mathbf{v}_{w \lambda}^{\vee} \in \mathbb{W}_{w}(\lambda)_{\mathbb{Z}}^{\vee}
$$

with paring 1 with $\mathbf{v}_{w \lambda} \in \mathbb{W}_{w}(\lambda)_{\mathbb{Z}}$. This vector $\mathbf{v}_{w \lambda}^{\vee}$ yields a $U_{\mathbb{k}}^{+}$-cocyclic vector of $\mathbb{W}_{w}(\lambda)_{\mathbb{k}}^{\vee}$ for a field $\mathbb{k}$. By the construction of the ring structure on $R_{w}$, we have $\mathbf{v}_{w w_{0} \lambda}^{\vee} \cdot \mathbf{v}_{w w_{0} \mu}^{\vee}=\mathbf{v}_{w w_{0}(\lambda+\mu)}^{\vee}$ in $R_{w}$ for every $\lambda, \mu \in P_{+}$.

Lemma 3.29. For each $w, v \in W_{\mathrm{af}}$ and $\mathrm{J} \subset \mathrm{I}$, the ring $R_{w}(\mathrm{~J})$ is a quotient of $R_{v}(\mathrm{~J})$ if $w \leq_{\frac{\infty}{2}} v$. In addition, the ring $R_{w}$ is a quotient of $R_{v}$ if and only if $w \leq_{\frac{\infty}{2}} v$.

Proof. We have $\mathbb{W}_{w w_{0}}(\lambda)_{\mathbb{Z}} \subset \mathbb{W}_{v w_{0}}(\lambda)_{\mathbb{Z}}$ if and only if $\mathbf{v}_{w w_{0} \lambda} \in \mathbb{W}_{v w_{0}}(\lambda)_{\mathbb{Z}}$. Now we apply Lemma 3.15 to deduce the result.

Lemma 3.30. We have morphisms of rings with $U_{\mathbb{Z}}^{+}$-actions

$$
R^{+} \longrightarrow R \hookrightarrow R^{\mathrm{af}},
$$

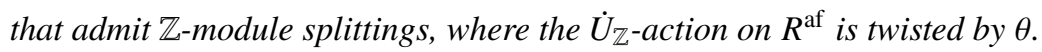

Proof. Apply Proposition 3.25 and Corollary 3.26.

For each $w \in W$ and $\mathrm{J} \subset \mathrm{I}$, we set

$$
\left(\mathbf{Q}_{G, \mathrm{~J}}(w)\right)_{\mathbb{Z}}:=\operatorname{Proj} R_{w}(\mathrm{~J}) \quad \text { and } \quad\left(\mathbf{Q}_{G, \mathrm{~J}}^{\mathrm{rat}}\right)_{\mathbb{Z}}:=\bigcup_{w \in W}\left(\mathbf{Q}_{G, \mathrm{~J}}(w)\right)_{\mathbb{Z}} .
$$

These schemes and ind-schemes are flat over $\mathbb{Z}$.

Remark 3.31. In view of equation (2.5), we have embeddings

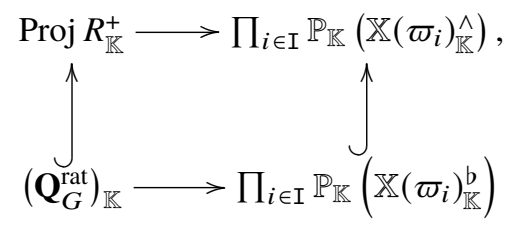

where we set

$$
\mathbb{X}\left(\varpi_{i}\right)_{\mathbb{K}}^{\wedge}:=\prod_{n \in \mathbb{Z}} \mathbb{X}\left(\varpi_{i}\right)_{n, \mathbb{K}} \text { and } \mathbb{X}\left(\varpi_{i}\right)_{\mathbb{K}}^{b}:=\bigcup_{m \in \mathbb{Z}} \prod_{n>m} \mathbb{X}\left(\varpi_{i}\right)_{n, \mathbb{K}}
$$

for each $i \in \mathrm{I}$. Here all the spaces in diagram (3.8) admit actions of $S L(2, i)_{\mathbb{K}}(i \in \mathrm{I})$, while only the bottom two spaces admit actions of $G[[z]]_{\mathbb{K}}$. Nevertheless, the top two spaces have some advantages, since they are $\theta$-stable (unlike the bottom two) and consequently also contain $\theta\left(\left(\mathbf{Q}_{G}^{\text {rat }}\right)_{\mathbb{K}}\right)$.

Theorem 3.32 ([49]). Let $p$ be a prime. Then the ring $R_{\mathbb{F}_{p}}^{\text {af }}$ admits a Frobenius splitting that is $\mathbf{I}$ - and $\mathbf{I}^{-}$-canonically split.

Theorem 3.33. Let $p$ be a prime. The ring $R_{\mathbb{F}_{p}}$ admits a Frobenius splitting that is $\mathbf{I}$-canonically split. 
Proof. The I-canonical Frobenius splitting $\phi$ of $R^{\text {af }}$ gives rise to the following maps, whose composition is the identity:

$$
L(\Lambda)_{\mathbb{F}_{p}} \stackrel{\phi^{\vee}}{\longrightarrow} L(p \Lambda)_{\mathbb{F}_{p}} \longrightarrow L(\Lambda)_{\mathbb{F}_{p}}, \quad \Lambda \in P_{+}^{\text {af }} .
$$

In view of Proposition 3.25, this prolongs to

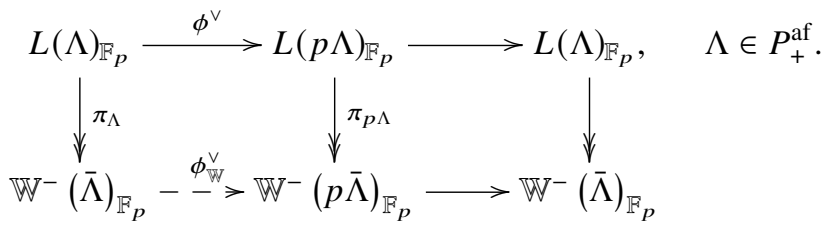

The right square is automatic (and is canonically defined) from the adjunction of the Frobenius pushforward (by taking the restricted dual). In order to show that $\phi$ descends to a Frobenius splitting of $R_{\mathbb{F}_{p}}$, it suffices to show that the dotted map $\phi_{\mathbb{W}}^{\vee}$ is a well-defined linear map (induced from $\phi^{\vee}$ and such that the left square is commutative).

By Corollary 3.24, $\operatorname{ker} \pi_{\Lambda}$ is generated by the $P$-weight $(P \backslash \operatorname{Conv} W \bar{\Lambda})$-part of $L(\Lambda)_{\mathbb{F}_{p}}$. By the cyclicity of $L(\Lambda)_{\mathbb{F}_{p}}$ as $U_{\mathbb{Z}}^{-}$-modules and Proposition 3.7, we deduce that $\phi^{\vee}\left(\operatorname{ker} \pi_{\Lambda}\right)$ is contained in the $U_{\mathbb{F}_{p}}^{-}$-submodule of $L(p \Lambda)_{\mathbb{F}_{p}}$ generated by the $P$-weight $p(P \backslash \operatorname{Conv} W \bar{\Lambda})$-part of $L(p \Lambda)_{\mathbb{F}_{p}}$. The latter is contained in ker $\pi_{p \Lambda}$ by

$$
p(P \backslash \operatorname{Conv} W \bar{\Lambda}) \subset P \backslash p \operatorname{Conv} W \bar{\Lambda} .
$$

Therefore, we conclude that $\phi_{\mathbb{W}}^{\vee}$ is a well-defined linear map, and hence $\theta^{*}\left(R_{\mathbb{F}_{p}}\right)$ admits a Frobenius splitting induced from $\phi$. The unipotent part of the $\mathbf{I}^{-}$-canonical splitting condition is in common with subrings. It remains to twist the grading given by $\alpha_{0}^{\vee}$ with that given by $-\vartheta^{\vee}$ and twist the $\mathbf{I}^{-}$-action into the I-action by $\theta$ to conclude that our Frobenius splitting on $R_{\mathbb{F}_{p}}$ is $\mathbf{I}$-canonical.

Corollary 3.34. Let $p$ be a prime and set $w \in W$. The $\mathbf{I}$-canonical splitting of $R_{\mathbb{F}_{p}}$ obtained in Theorem 3.33 induces an $\mathbf{I}$-canonical splitting of $\left(R_{w}\right)_{\mathbb{F}_{p}}$.

Proof. We set $L^{w w_{0}}(\Lambda)_{\mathbb{Z}}:=U_{\mathbb{Z}}^{-} \mathbf{v}_{w w_{0} \Lambda}$, where $\mathbb{Z} \mathbf{v}_{w w_{0} \Lambda}$ is the $P^{\text {af }}$-weight $w w_{0} \Lambda$-part of $L(\Lambda)_{\mathbb{Z}}$, which has rank 1 over $\mathbb{Z}$.

The subspace $\mathbb{W}_{w w_{0}}^{-}(\lambda)_{\mathbb{Z}} \subset \mathbb{W}^{-}(\lambda)_{\mathbb{Z}}$ is the image of $L^{w w_{0}}(\Lambda)_{\mathbb{Z}} \subset L(\Lambda)_{\mathbb{Z}}($ with $\lambda=\bar{\Lambda})$ under Theorem 3.23 , since $L^{w w_{0}}(\Lambda)_{\mathbb{Z}}$ is spanned by a subset of $\mathbf{B}(\Lambda)([43,(0.3)])$. Our Frobenius splitting $\phi$ is obtained from that of $R_{\mathbb{F}_{p}}^{\text {af }}$, which is compatible with $\bigoplus_{\lambda \in P_{+}} L^{w w_{0}}(\Lambda)_{\mathbb{F}_{p}}^{\vee}$ by [49, Corollary B]. Applying $\theta$, we conclude that $\phi$ must descend to a Frobenius splitting of $\left(R_{w}\right)_{\mathbb{F}_{p}}$.

Corollary 3.35. An I-canonical splitting of $R_{\mathbb{F}_{p}}$ is unique.

Proof. The behaviour of the vectors in formula (3.7) (with $w=e$ ) under an I-canonical Frobenius splitting is uniquely determined, as they form a polynomial ring isomorphic to $\mathbb{F}_{p} P_{+}$such that each of its $\left(P_{+^{-}}\right)$graded components is a multiplicity-free $P^{\text {af }}$-weight space in $\mathbb{W}(\lambda)_{\mathbb{F}_{p}}^{\vee}$ s. By Proposition 3.7, this completely determines the behaviour of our splitting (through its dual map).

Corollary 3.36. An I-canonical splitting of $R_{\mathbb{F}_{p}}$ is compatible with $\left(R_{w}\right)_{\mathbb{F}_{p}}$ for every $w \in W_{\text {af }}$ such that $w \leq \frac{\infty}{2} e$.

Proof. By [14, Proposition 4.1.17 and Remark 4.1.18 (i)] and [46, Theorem 4.12] (the algebraic portion of the latter stemming from [45, Lemma 2.6], which carries over to this setting; compare [46, Lemma 4.4 and Theorem 4.7]), we derive that an I-canonical Frobenius splitting of $\left(R_{w}\right)_{\mathbb{F}_{p}}(w \in W)$ gives rise 
to an I-canonical splitting of $R_{\mathbb{F}_{p}}$ that is compatible with $\left(R_{w}\right)_{\mathbb{F}_{p}}$ (arguing by restricting to the $S L(2, i)$ actions for each $i \in \mathrm{I})$. In particular, the I-canonical splitting of $\left(R_{w}\right)_{\mathbb{F}_{p}}(w \in W)$ also uniquely exists and is compatible with that of $R_{\mathbb{F}_{p}}$ by Corollaries 3.34 and 3.35. By Corollary 3.28, we further deduce that the I-canonical splitting of $\left(R_{w}\right)_{\mathbb{F}_{p}}\left(w \in W_{\text {af }}\right)$ uniquely exists.

Set $w \in W$ such that $s_{0} w=s_{\vartheta} w t_{-w^{-1}} \vartheta^{\vee} \leq_{\frac{\infty}{2}} w$. Then $R_{s_{0} w}$ is a quotient of $R_{w}$. Again by [14, Proposition 4.1.17 and Remark 4.1.18 (i)], the set of $\mathbf{I}$-canonical splittings of $\left(R_{s_{0} w}\right)_{\mathbb{F}_{p}}$ is in bijection with that of $\left(R_{w}\right)_{\mathbb{F}_{p}}$ that are compatible with $\left(R_{S_{0} w}\right)_{\mathbb{F}_{p}}$. By Corollary 3.28 and the previous paragraph, we find that the (unique) I-canonical splitting of $\left(R_{S_{0} w}\right)_{\mathbb{F}_{p}}$ is compatible with $\left(R_{w}\right)_{\mathbb{F}_{p}}$, and hence also compatible with $R_{\mathbb{F}_{p}}$ and $\left(R_{t_{-w^{-1} \vartheta} \vee}\right)_{\mathbb{F}_{p}}$. This forces the I-canonical splitting of $R_{\mathbb{F}_{p}}$ to be compatible with that of $\left(R_{u t_{-w^{-1}} \vartheta^{\vee}}\right)_{\mathbb{F}_{p}}$ for every $u, w \in W$ such that $-w^{-1} \vartheta^{\vee} \in \Delta_{+}^{\vee}$.

The set $\left(\left\{-w^{-1} \vartheta^{\vee}\right\}_{w \in W} \cap Q_{+}^{\vee}\right)$ is precisely the set of short positive coroots in $\Delta_{+}^{\vee}$. This spans $Q_{+}^{\vee}$ as monoids, by inspection. Since $w \in W_{\mathrm{af}}$ with $w \leq_{\frac{\infty}{2}} e$ is written as $w=u t_{\beta}$ for some $u \in W$ and $\beta \in Q_{+}^{\vee}$ ([74, Lecture 13, Proposition 1]), we conclude the assertion.

Theorem 3.37. Let $p$ be a prime and set $\mathrm{J} \subset \mathrm{I}$. The ring $R^{+}(\mathrm{J})_{\mathbb{F}_{p}}$ admits a Frobenius splitting that is I- and $\mathbf{I}^{-}$-canonically split. This splitting is compatible with $R_{w}(\mathrm{~J})_{\mathbb{F}_{p}}$ and the image of $R^{+}(\mathrm{J})_{\mathbb{F}_{p}} \subset R_{\mathbb{F}_{p}}^{+}$ under the quotient map $R_{\mathbb{F}_{p}}^{+} \rightarrow \theta^{*}\left(\left(R_{w w_{0}}\right)_{\mathbb{F}_{p}}\right)$ for each $w \in W_{\mathrm{af}}$.

Proof. Since the case of $\mathrm{J} \neq \emptyset$ follows by the restriction to a part of the $P_{+}$-grading, we concentrate on the case where $\mathrm{J}=\emptyset$.

The ring structure of $R_{\mathbb{F}_{p}}^{+}$is determined by $R_{\mathbb{F}_{p}}$ through the application of $U_{\mathbb{Z}}^{-}$before taking duals. By Corollary 3.36 (and its proof), it defines an $\mathbf{I}$-canonical splitting $\phi$ of $R_{\mathbb{F}_{p}}^{+}$compatible with $\left(R_{w}\right)_{\mathbb{F}_{p}}$ for each $w \in W_{\mathrm{af}}$. The ring $R_{\mathbb{F}_{p}}^{+}$admits an $S L(2, i)_{\mathbb{F}_{p}}$-action that integrates the actions of $E_{i}^{(n)}$ and $F_{i}^{(n)}$ ( $n \in \mathbb{Z}_{>0}$ ) for each $i \in \mathrm{I}_{\mathrm{af}}$. By [14, Proposition 2.10], this splitting $\phi$ is also $\mathbf{I}^{-}$-canonical.

The I-cocyclic $P^{\text {af }}$-weight vector $\mathbf{v}_{w w_{0} \lambda}^{\vee} \in \mathbb{W}_{w w_{0}}(\lambda)_{\mathbb{F}_{p}}^{\vee}$ is uniquely characterised by its $P^{\text {af }}$-weight. Hence we obtain a map

$$
\mathbb{X}(\lambda)_{\mathbb{F}_{p}}^{\vee} \supset \operatorname{Span}_{\mathbb{Z}} \prod_{i \in \mathrm{I}}\left(\mathbb{X}\left(\varpi_{i}\right)_{\mathbb{F}_{p}}^{\vee}\right)^{\left\langle\alpha_{i}, \lambda\right\rangle} \longrightarrow \mathbb{W}_{w w_{0}}(\lambda)_{\mathbb{F}_{p}}^{\vee} \longrightarrow \mathbb{F}_{p} \mathbf{v}_{w w_{0} \lambda}^{\vee}
$$

It gives rise to the ring surjections

$$
R_{\mathbb{F}_{p}}^{+} \longrightarrow\left(R_{w}\right)_{\mathbb{F}_{p}} \longrightarrow \bigoplus_{\lambda \in P_{+}} \mathbb{F}_{p} \mathbf{v}_{w w_{0} \lambda}^{\vee}
$$

which are compatible with $\phi$ by construction in the first surjection and by examining the $P^{\text {af }}$-weights in the second (we denote this composition surjective ring map by $\xi$ ). Consider the ideal

$$
I(w):=R_{\mathbb{F}_{p}}^{+} \cap \bigcap_{g \in \mathbf{I}^{-}\left(\overline{\mathbb{F}}_{p}\right)} g\left(\overline{\mathbb{F}}_{p} \otimes_{\mathbb{F}_{p}} \operatorname{ker} \xi\right) \subset R_{\overline{\mathbb{F}}_{p}}^{+} .
$$

(Here the action of $\mathbf{I}^{-}\left(\overline{\mathbb{F}}_{p}\right)$ is obtained by the unipotent one-parameter subgroups $\left\{\rho_{-\alpha_{i}}\right\}_{i \in \mathrm{I}_{\mathrm{af}}}$ defined through the exponentials, which are well defined, as we have all the divided powers. The passage from $\mathbb{F}_{p}$ to $\overline{\mathbb{F}}_{p}$ is necessary to ensure scheme-theoretic invariance, since we want an intersection that is equivalent to the geometric $\mathbb{G}_{a}$-actions through $\rho_{-\alpha_{i}}$ for all $i \in \mathrm{I}_{\mathrm{af}}$ by finding the Zariski dense subsets of $\mathbb{G}_{a}$. For this purpose, we want the image of each $\rho_{-\alpha_{i}}$ to be infinite, which cannot be achieved by sending $\mathbb{G}_{a}\left(\mathbb{F}_{p}\right) \cong \mathbb{F}_{p}$.) This ideal is the maximal $U_{\mathbb{F}_{p}}^{-}$-invariant ideal of $R_{\mathbb{F}_{p}}^{+}$that is contained in ker $\xi$. 
Let us denote the quotient ring by

$$
Q=\bigoplus_{\lambda \in P_{+}} Q(\lambda):=R_{\mathbb{F}_{p}}^{+} / I(w) .
$$

By the construction of $I(w)$, we deduce that

$$
U_{\mathbb{F}_{p}}^{-} \mathbf{v}_{w w_{0} \lambda} \subset Q(\lambda)^{\vee} \subset \mathbb{X}(\lambda)_{\mathbb{F}_{p}}
$$

for each $\lambda \in P_{+}$(otherwise we can differentiate a vector in $I(w)$ to obtain a nonzero element of $\left.\bigoplus_{\lambda \in P_{+}} \mathbb{F}_{p} \mathbf{v}_{w w_{0} \lambda}^{\vee}\right)$. Since $\theta^{*}\left(\mathbb{W}_{w w_{0}}\left(-w_{0} \lambda\right)_{\mathbb{F}_{p}}\right)$ is a cyclic $U_{\mathbb{F}_{p}}^{-}$-submodule of $\mathbb{X}(\lambda)_{\mathbb{F}_{p}}$, we have $U_{\mathbb{F}_{p}}^{-} \mathbf{v}_{w w_{0} \lambda}=\theta^{*}\left(\mathbb{W}_{w w_{0}}\left(-w_{0} \lambda\right)_{\mathbb{F}_{p}}\right)$. In particular, we deduce a vector space surjection

$$
R_{\mathbb{F}_{p}}^{+} / I(w) \longrightarrow \theta^{*}\left(\left(R_{w w_{0}}\right)_{\mathbb{F}_{p}}\right)
$$

(compare Corollary 3.26). Since the right-hand side is naturally a ring, we conclude

$$
R_{\mathbb{F}_{p}}^{+} / I(w) \cong \theta^{*}\left(\left(R_{w w_{0}}\right)_{\mathbb{F}_{p}}\right)
$$

by the maximality of $I(w)$.

The ideal $I(w) \subset R_{\mathbb{F}_{p}}^{+}$also splits compatibly by $\phi$ (since $\phi$ is $\mathbf{I}^{-}$-canonical and ker $\xi$ splits compatibly). In particular, each $\theta^{*}\left(\left(R_{w w_{0}}\right)_{\mathbb{F}_{p}}\right)$ compatibly splits under $\phi$, as required.

Corollary 3.38. For each $\mathrm{J} \subset \mathrm{I}$, the ind-scheme $\left(\mathbf{Q}_{G, \mathrm{~J}}^{\text {rat }}\right)_{\mathbb{F}_{p}}$ admits an $\mathbf{I}$ - and $\mathbf{I}^{-}$-canonical Frobenius splitting that is compatible with $\mathbf{Q}_{G, \mathrm{~J}}(w)_{\mathbb{F}_{p}}$ for each $w \in W_{\mathrm{af}}$.

Proof. The condition of the canonical splitting can be checked by line bundle twists, and hence we only need to show whether the Frobenius splitting of $R_{w}(\mathrm{~J})_{\mathbb{F}_{p}}$ descends to $\mathbf{Q}_{G, \mathrm{~J}}(w)_{\mathbb{F}_{p}}$ for each $w \in W_{\mathrm{af}}$. Since $\mathbf{Q}_{G, \mathrm{~J}}(w)_{\mathbb{F}_{p}}$ is the quotient of an open subset of $X:=\operatorname{Spec} R_{w}(\mathrm{~J})_{\mathbb{F}_{p}}$ by $H$ (corresponding to the $P_{\mathrm{J},+}$-grading), and our Frobenius splitting is $H$-fixed, it suffices to see whether the localisation to the nonirrelevant locus preserves the Frobenius splitting. This follows if the localisation of $\mathrm{Fr}_{*} \mathcal{O}_{X}=\mathcal{O}_{X^{(1)}}$ as a $\mathrm{Fr}_{*} \mathcal{O}_{X}$-module and as an $\mathcal{O}_{X}$-module are the same. This holds, as localisation by a multiplicative set $S$ is the same as localisation by $S^{p}$.

Recall that a scheme $X$ defined over a field $\mathbb{k}$ is called weakly normal if every finite bijective birational $(\mathbb{k}$-)morphism $f: Y \rightarrow X$ from a (reduced) scheme over $\mathbb{k}$ is in fact an isomorphism ([68] and [14, $\S 1.2 .3])$.

Corollary 3.39. For each $\mathrm{J} \subset \mathrm{I}$, the ind-scheme $\left(\mathbf{Q}_{G, \mathrm{~J}}^{\mathrm{rat}}\right)_{\mathbb{F}_{p}}$ and the schemes $\mathbf{Q}_{G, \mathrm{~J}}(w)_{\mathbb{F}_{p}}\left(w \in W_{\mathrm{af}}\right)$ are reduced. In addition, $\mathbf{Q}_{G, \mathrm{~J}}(w)_{\mathbb{F}_{p}}$ is weakly normal.

Proof. For the first assertion, apply [14, Proposition 1.2.1] to Corollary 3.38. For the second assertion, apply [14, Proposition 1.2.5] to Corollary 3.34.

\section{Frobenius splitting of quasi-map spaces}

We retain the settings of the previous section. In particular, we sometimes work over a ring or a nonalgebraically closed field. Moreover, the notational conventions explained in the beginning of Section 3 continue to apply. 


\subsection{The scheme $\mathbb{Q}_{j}^{\prime}(v, w)$ and its Frobenius splitting}

Set $v, w \in W_{\text {af }}$ and $\mathrm{J} \subset \mathrm{I}$. We set

$$
R_{w}^{v}(\mathrm{~J}):=R^{+}(\mathrm{J}) /\left(\operatorname{ker}\left(R^{+}(\mathrm{J}) \rightarrow R_{w}(\mathrm{~J})\right)+\operatorname{ker}\left(R^{+}(\mathrm{J}) \rightarrow \theta^{*}\left(R_{v w_{0}}\left(\mathrm{~J}^{\prime}\right)\right)\right)\right),
$$

where we have $\operatorname{ker}\left(R^{+}(\mathrm{J}) \rightarrow \theta^{*}\left(R_{v w_{0}}\left(\mathrm{~J}^{\prime}\right)\right)\right)=\operatorname{ker}\left(R^{+}(\mathrm{J}) \rightarrow \theta^{*}\left(R_{v w_{0}}\right)\right)$ for

$$
\mathrm{J}^{\prime}:=\left\{i \in \mathrm{I} \mid-w_{0} \alpha_{i}=\alpha_{j}, \exists j \in \mathrm{J}\right\} \subset \mathrm{I} .
$$

By construction, $R_{w}^{v}(\mathrm{~J})$ is a $P_{\mathrm{J},+}$-graded ring. We set

$$
R_{w}^{v}(\mathrm{~J}):=\bigoplus_{\lambda \in P_{\mathrm{J},+}} R_{w}^{v}(\mathrm{~J}, \lambda)
$$

Lemma 4.1. For each $w, v \in W_{\mathrm{af}}$ and $\mathrm{J} \subset \mathrm{I}$, the multiplication map

$$
R_{w}^{v}(\mathrm{~J}, \lambda) \otimes_{\mathbb{Z}} R_{w}^{v}(\mathrm{~J}, \mu) \rightarrow R_{w}^{v}(\mathrm{~J}, \lambda+\mu), \quad \lambda, \mu \in P_{\mathrm{J},+},
$$

is surjective.

Proof. We have a quotient

$$
R_{w}(\mathrm{~J})=\bigoplus_{\lambda \in P_{\mathrm{J},+}} \mathbb{W}_{w w_{0}}(\lambda)_{\mathbb{Z}}^{\vee} \longrightarrow \bigoplus_{\lambda \in P_{\mathrm{J},+}} R_{w}^{v}(\mathrm{~J}, \lambda)=R_{w}^{v}(\mathrm{~J})
$$

of homogeneous rings. Corollary 3.26 implies that the multiplication map of $R_{w}(\mathrm{~J})$ is surjective. Hence so is the quotient ring.

We set

$$
Q_{\jmath}^{\prime}(v, w):=\operatorname{Proj} R_{w}^{v}(\mathrm{\jmath})
$$

where our definition of Proj is equation (2.5). In case where $v=w_{0} t_{\beta}$ for $\beta \in Q^{\vee}$, we may write $R_{w}^{v}(\mathrm{~J})$ and $Q_{\mathrm{J}}^{\prime}(v, w)$ as $R_{w}^{\beta}(\mathrm{J})$ and $Q_{\mathrm{J}}^{\prime}(\beta, w)$, respectively.

Lemma 4.2. For each $w, v \in W$, the Chevalley involution induces an isomorphism

$$
\mathcal{Q}^{\prime}(v, w) \stackrel{\cong}{\longrightarrow} Q^{\prime}\left(w w_{0}, v w_{0}\right) .
$$

Proof. Apply Lemma 3.17 to the construction of $R_{w}^{v}$ to deduce an isomorphism

$$
\theta^{*}: R_{w}^{v} \stackrel{\cong}{\longrightarrow} R_{v w_{0}}^{w w_{0}}
$$

of graded rings, which yields the assertion.

Lemma 4.3. For each $w, v \in W_{\text {af }}$ and $\mathrm{J} \subset \mathrm{I}$, the scheme $Q_{\mathrm{J}}^{\prime}(v, w)$ is flat over $\mathbb{Z}$.

Proof. The ring $R^{+}(\mathrm{J})$ has a $\mathbb{Z}$-basis that is dual to $\bigsqcup_{\lambda \in P_{\mathrm{J},+}} \mathbf{B}(\mathbb{X}(\lambda))$. The rings $R_{w}(\mathrm{~J})$ and $\operatorname{Im}\left(R^{+}(\mathrm{J}) \rightarrow \theta^{*}\left(R_{v w_{0}}\right)\right)$ are quotients by subsets of such basis elements, by Lemma 3.17. Hence we have a free $\mathbb{Z}$-basis of $R_{w}^{v}(\mathrm{~J})$, as required.

Lemma 4.4. For each $w, v \in W_{\mathrm{af}}$ and $\mathrm{J} \subset \mathrm{I}$, the scheme $\mathbb{Q}_{\mathrm{J}}^{\prime}(v, w)$ is projective (of finite type) over $\mathbb{Z}$.

Proof. By [44, Proposition 5.16], we have $\operatorname{rank}_{\mathbb{Z}} R_{w}^{v}\left(\varpi_{i}\right)<\infty$ for each $i \in \mathrm{I}$. By Lemma 4.1, this forces $Q_{j}^{\prime}(v, w)$ to be a projective scheme (which usually implies finite type by definition; here we explicitly include it, since our Proj does not yield a finite-type scheme in general), as required. 
Lemma 4.5. Set $\mathrm{J} \subset \mathrm{I}$. We have $\mathbb{Q}_{\mathrm{J}}^{\prime}(v, w) \neq \emptyset$ if $v \leq_{\frac{\infty}{2}} w$.

Proof. We have $R_{w}^{v}(\mathrm{~J}, \lambda) \neq\{0\}$ if $\mathbf{v}_{v w_{0} \lambda} \in \mathbb{W}_{w w_{0}}(\lambda)_{\mathbb{Z}}$ by Lemma 3.17(4). Here $\mathbf{v}_{v w_{0} \lambda} \in \mathbb{W}_{w w_{0}}(\lambda)_{\mathbb{Z}}$ is equivalent to $\mathbb{W}_{v w_{0}}(\lambda)_{\mathbb{Z}} \subset \mathbb{W}_{w w_{0}}(\lambda)_{\mathbb{Z}}$. Now apply Lemma 3.15 to obtain the assertion.

Lemma 4.6. Set $v, w \in W_{\mathrm{af}}, \beta \in Q^{\vee}$ and $\mathrm{J} \subset \mathrm{I}$. We have $\mathbb{Q}_{\mathrm{J}}^{\prime}(v, w) \cong \mathbb{Q}_{\mathrm{J}}^{\prime}\left(v t_{\beta}, w t_{\beta}\right)$.

Proof. We borrow notation from Lemma 3.17. By the definition of our ring $R_{w}^{v}(\mathrm{~J})$, the assertion follows if

$$
\mathbb{W}_{w t_{\beta} w_{0}}(\lambda)_{\mathbb{Z}}=\tau_{w_{0} \beta} \mathbb{W}_{w w_{0}}(\lambda)_{\mathbb{Z}} \quad \text { and } \quad \tau_{w_{0} \beta} \theta^{*}\left(\mathbb{W}_{v}\left(-w_{0} \lambda\right)_{\mathbb{Z}}\right)=\theta^{*}\left(\mathbb{W}_{v t_{\beta}}\left(-w_{0} \lambda\right)_{\mathbb{Z}}\right)
$$

hold for each $w, v \in W_{\mathrm{af}}, \beta \in Q^{\vee}$ and $\lambda \in P_{+}$. These assertions themselves follow by chasing the weights of the cyclic vectors.

Lemma 4.7. Let $p$ be a prime and set $\mathrm{J} \subset \mathrm{I}$. For each $w, v \in W_{\mathrm{af}}$, the ring $R_{w}^{v}(\mathrm{~J})_{\mathbb{F}_{p}}$ admits a Frobenius splitting that is compatible with the quotient $R_{w^{\prime}}^{v^{\prime}}(\mathrm{J})_{\mathbb{F}_{p}}$ for $v^{\prime}, w^{\prime} \in W_{\text {af }}$ such that $v \leq_{\frac{\infty}{2}} v^{\prime} \leq_{\frac{\infty}{2}} w^{\prime} \leq_{\frac{\infty}{2}} w$. In particular, the scheme $Q_{\mathrm{J}}^{\prime}(v, w)_{\mathbb{F}_{p}}$ is reduced and weakly normal.

Proof. By construction, $\operatorname{ker}\left(R^{+}(\mathrm{J})_{\mathbb{F}_{p}} \rightarrow\left(R_{w}\right)_{\mathbb{F}_{p}}\right)$ and $\operatorname{ker}\left(R^{+}(\mathrm{J})_{\mathbb{F}_{p}} \rightarrow \theta^{*}\left(\left(R_{v w_{0}}\right)_{\mathbb{F}_{p}}\right)\right)$ are ideals of a ring $R^{+}(\mathrm{J})_{\mathbb{F}_{p}}$ that are compatible with the canonical Frobenius splitting of $R^{+}(\mathrm{J})_{\mathbb{F}_{p}}$ by Theorem 3.37. Hence so is their sum. It must be compatible with every quotient of the form $R_{w^{\prime}}^{v^{\prime}}(\mathrm{J})_{\mathbb{F}_{p}}$ with the given condition, Corollary 3.34 and Lemmas 3.17(4), 3.29 and 4.5. This proves the first assertion. We apply [14, Proposition 1.2.1 and 1.2.5] to deduce the second assertion.

Corollary 4.8. Let p be a prime. For each $w, v \in W$, the scheme $Q_{j}^{\prime}(v, w)_{\mathbb{F}_{p}}$ admits a Frobenius splitting compatible with $Q_{\mathrm{J}}^{\prime}\left(v^{\prime}, w^{\prime}\right)_{\mathbb{F}_{p}}$ for every $w^{\prime}, v^{\prime} \in W_{\mathrm{af}}$ such that $v \leq_{\frac{\infty}{2}} v^{\prime} \leq_{\frac{\infty}{2}} w^{\prime} \leq_{\frac{\infty}{2}} w$.

Proof. Apply Theorem 3.5 to Lemma 4.7.

Remark 4.9. Unlike the case of Corollary 3.38, the space Spec $R_{w}^{v}(\mathrm{~J})_{\mathbb{F}_{p}}$ is not irreducible in general. In fact, we discard some of its irreducible components from Lemma 4.7 to Corollary 4.8.

Corollary 4.10. The ring $R_{w}^{v}(\mathrm{~J})$ is reduced. In particular, the scheme $\mathscr{Q}_{\mathrm{J}}^{\prime}(v, w)$ is reduced.

Proof. By Lemma 4.3, every nonzero element of $R_{w}^{v}(\mathrm{~J})$ is annihilated by reduction mod $p$ for only finitely many primes. Now it remains to apply Lemma 4.7 .

\subsection{Modular interpretation of $\mathrm{Q}_{G, \mathrm{~J}}^{\mathrm{rat}}$}

We have an identification

$$
W_{\mathrm{af}} \cong N_{G((z))}(H(\mathbb{K})) / H(\mathbb{K})
$$

regardless of the (algebraically closed) base field $\mathbb{K}$. We denote a lift of $w \in W_{\text {af }}$ in $N_{G((z))}(H(\mathbb{K})) / H(\mathbb{K})$ by $\dot{w}$.

Lemma 4.11. For each $w \in W$ and $\mathrm{J} \subset \mathrm{I}$, the scheme $\mathbf{Q}_{G, \mathrm{~J}}(w)_{\mathbb{K}}$ contains an affine Zariski open $\mathbf{I}_{\mathbb{K}}$-orbit $\mathbb{O}(\mathrm{J}, w)_{\mathbb{K}}$ that is isomorphic to

$$
\mathbf{I}_{\mathbb{K}} /\left(H_{\mathbb{K}} \cdot\left(\operatorname{Ad}\left(\dot{w} \dot{w}_{0}\right)([P(\mathrm{~J}), P(\mathrm{~J})]((z))) \cap \mathbf{I}_{\mathbb{K}}\right)\right)
$$

as a scheme over $\mathbb{K}$. (By abuse of notation, here we identify the set of $\mathbb{K}$-valued points $\left(\operatorname{Ad}\left(\dot{w} \dot{w}_{0}\right)([P(\mathrm{~J}), P(\mathrm{~J})]((z))) \cap \mathbf{I}_{\mathbb{K}}\right)$ with its Zariski closure in $\mathbf{I}_{\mathbb{K}}$.) It is an open neighbourhood of the unique $\left(H \times \mathbb{G}_{m}\right)_{\mathbb{K}}$-fixed point of $\mathbb{O}(\mathrm{J}, w)_{\mathbb{K}}$. 
Proof. Recall that $\mathbf{v}_{w w_{0} \lambda}^{\vee} \cdot \mathbf{v}_{w w_{0} \mu}^{\vee}=\mathbf{v}_{w w_{0}(\lambda+\mu)}^{\vee}$ for each $\lambda, \mu \in P_{+}$. Since $\mathbb{W}_{w w_{0}}(\lambda)$ is compatible with the positive global basis, the ring

$$
\mathbb{Z}[w]:=\sum_{\lambda \in P_{\mathrm{J},+}}\left(\mathbf{v}_{w w_{0} \lambda}^{\vee}\right)^{-1} \mathbb{W}_{w w_{0}}(\lambda)_{\mathbb{Z}}^{\vee} \subset\left(U_{\mathbb{Z}}^{+}\right)^{\vee}
$$

admits its dual basis. By construction, $\mathbb{Z}[w]$ is the coordinate ring of an affine Zariski open set of $\mathbf{Q}_{G}(w)_{\mathbb{Z}}$. In addition, it inherits a natural $P^{\text {af }}$-grading from $R_{w}(\mathrm{~J})$. Therefore, $\mathbb{Z}[w]$ defines an open neighbourhood of an $\left(H \times \mathbb{G}_{m}\right)$-fixed point of $\mathbf{Q}_{G, J}(w)$ obtained by the linear forms $\left\{\mathbf{v}_{w w_{0} \lambda}\right\}_{\lambda}$. We set $\mathbb{C}[w]:=\mathbb{C} \otimes_{\mathbb{Z}} \mathbb{Z}[w]$ and $\mathbb{K}[w]:=\mathbb{K} \otimes_{\mathbb{Z}} \mathbb{Z}[w]$.

The defining relation of $(\mathbb{C}[e])^{\vee}$ in terms of the $U_{\mathbb{C}}^{+}$-action is

$$
U_{\mathbb{C}}^{+}\left(\operatorname{Ad}\left(\dot{w}_{0}\right)([\mathfrak{p}(J), \mathfrak{p}(J)]) \otimes \mathbb{C}[z] \cap \mathfrak{I}\right) .
$$

by (the limit of) Chari, Fourier and Khandai [17, Proposition 3.3]. Since $\mathbb{W}_{u w_{0}}(\lambda)_{\mathbb{Z}} \subset \mathbb{W}_{w_{0}}(\lambda)_{\mathbb{Z}}$ for each $u \in W$, the defining relation of $(\mathbb{C}[u])^{\vee}$ in terms of the $U_{\mathbb{C}}^{+}$-action is

$$
U_{\mathbb{C}}^{+}\left(\operatorname{Ad}\left(\dot{u} \dot{w}_{0}\right)([\mathfrak{p}(J), \mathfrak{p}(J)]) \otimes \mathbb{C}[z] \cap \mathfrak{I}\right)
$$

by applying the action of $\dot{u} \in N_{G}(H)_{\mathbb{C}}$ that lifts $u \in W$. In particular, we have

$$
\operatorname{Spec} \mathbb{C}[w] \cong \mathbf{I}_{\mathbb{C}} /\left(H_{\mathbb{C}} \cdot \operatorname{Ad}\left(\dot{w} \dot{w}_{0}\right)\left(\left[P(J)_{\mathbb{C}}, P(J)_{\mathbb{C}}\right][[z]]\right) \cap \mathbf{I}_{\mathbb{C}}\right)
$$

as schemes over $\mathbb{C}$. We put $\mathbf{I}_{\mathbb{Z}}^{1}:=\left[\mathbf{I}_{\mathbb{Z}}, \mathbf{I}_{\mathbb{Z}}\right]$. Let $\mathbf{L}_{\mathbb{C}}$ and $\mathbf{R}_{\mathbb{C}}$ be prounipotent subgroups of $\mathbf{I}_{\mathbb{C}}^{1}$ whose closed points are

$$
\text { Specm } \mathbb{C}[w] \text { and } \operatorname{Ad}\left(\dot{w} \dot{w}_{0}\right)\left(\left[P(J)_{\mathbb{C}}, P(J)_{\mathbb{C}}\right][[z]]\right) \cap \mathbf{I}_{\mathbb{C}}^{1},
$$

respectively, and they are stable by the natural $\left(H \times \mathbb{G}_{m}\right)_{\mathbb{C}}$-action on $\mathbf{I}_{\mathbb{C}}$. The isomorphism (4.2) gives rise to an isomorphism

$$
m_{\mathbb{C}}: \mathbf{L}_{\mathbb{C}} \times \mathbf{R}_{\mathbb{C}} \stackrel{\cong}{\longrightarrow} \mathbf{I}_{\mathbb{C}}^{1}
$$

of schemes over $\mathbb{C}$, where $m_{\mathbb{C}}$ is the multiplication map, as all the groups are prounipotent and the isomorphism between the sets of closed points is compatible with truncations by $P^{\text {af }}$-weight considerations. The Hopf algebra $\mathbb{Z}\left[\mathbf{I}_{\mathbb{Z}}^{1}\right]:=\left(U_{\mathbb{Z}}^{+}\right)^{\vee}$ is the coordinate ring of $\mathbf{I}_{\mathbb{Z}}^{1}$ (compare [49, Theorem 1.3]). Sending $\mathbb{Z}\left[\mathbf{I}^{1}\right]$ by the restriction morphisms $\mathbb{C}\left[\mathbf{I}_{\mathbb{C}}^{1}\right] \rightarrow \mathbb{C}\left[\mathbf{L}_{\mathbb{C}}\right]$ and $\mathbb{C}\left[\mathbf{I}_{\mathbb{C}}^{1}\right] \rightarrow \mathbb{C}\left[\mathbf{R}_{\mathbb{C}}\right]$, we have the corresponding group schemes $\mathbf{L}_{\mathbb{Z}}$ and $\mathbf{R}_{\mathbb{Z}}$ over $\mathbb{Z}$ (we denote their coordinate rings as $\mathbb{Z}[\mathbf{L}]$ and $\mathbb{Z}[\mathbf{R}]$, respectively). For each real root $\alpha \in \Delta_{\mathrm{af},+}$, one of the restricted dual rings $\mathbb{C}\left[\mathbf{L}_{\mathbb{C}}\right]^{\vee}$ or $\mathbb{C}\left[\mathbf{R}_{\mathbb{C}}\right]^{\vee}$ contains the image of a primitive element of $U_{\mathbb{Z}}^{+}$with $\widetilde{\mathfrak{h}}$-weight $\alpha$ (obtained by conjugations of $\left\{E_{i}\right\}_{i \in \mathrm{I}_{\mathrm{af}}} \mathrm{s}$; compare [66, Proposition 40.1.3] or [31, Lemma 6.6]), and hence the corresponding one-parameter subgroup lands in either $\mathbf{L}_{\mathbb{Z}}$ or $\mathbf{R}_{\mathbb{Z}}$. In view of [5, Theorem 3.13] (or [31, Theorem 5.8]), they generate a closed normal subgroup scheme $\mathbf{N}_{\mathbb{Z}}^{-}$of $\mathbf{L}_{\mathbb{Z}}$ that is a projective limit of extensions of $\mathbb{G}_{a}$ over $\mathbb{Z}$. (The same is true and exhausts the whole of $\mathbf{R}_{\mathbb{Z}}$ if $\mathrm{J}=\emptyset$.)

We examine the action of the imaginary Poincaré-Birkhoff-Witt generators $\left\{\tilde{P}_{i, m \delta}\right\}_{i \in \mathrm{I} \backslash \mathrm{J}, m>0}$ (the weight of $\tilde{P}_{i, m \delta}$ is $m \delta$ ) in [5, (3.7)]. By applying them on the direct sum of $P$-weight $\lambda$-parts of $\mathbb{X}(\lambda)_{\mathbb{C}}$ for all $\lambda \in P_{\mathrm{J}_{+}}$, we obtain a quotient group scheme $\mathbf{L}_{\mathbb{Z}} \rightarrow \mathbf{T}_{\mathbb{Z}}$ whose kernel is $\mathbf{N}_{\mathbb{Z}}^{-}$. By [5, Proposition 3.22], the group scheme $\operatorname{Spec} \mathbb{Z}\left[\tilde{P}_{i, m \delta} \mid m>1\right]^{\vee}$ for each $i \in \mathrm{I}$ is isomorphic to a projective limit of extensions of $\mathbb{G}_{a}$ (given by truncations with respect to the duals of $\left\{\tilde{P}_{i, m \delta}\right\}_{m>N}$ for $N \in \mathbb{Z}_{>0}$ ), which is flat over $\mathbb{Z}$. Thus so is $\mathbf{T}_{\mathbb{Z}}$.

From these, we deduce that $\mathbf{L}_{\mathbb{Z}} \subset \mathbf{I}_{\mathbb{Z}}^{1}$ defines a group subscheme over $\mathbb{Z}$ such that $\mathbb{Z}\left[\mathbf{I}_{\mathbb{Z}}^{1}\right] \rightarrow \mathbb{Z}[\mathbf{L}]$ splits as $\mathbb{Z}$-modules. The $P$-weight decomposition of the coordinate ring defines a splitting $\mathbf{T}_{\mathbb{Z}} \subset \mathbf{L}_{\mathbb{Z}} \subset \mathbf{I}_{\mathbb{Z}}^{1}$. 
Since $[L(J), L(J)]$ defines a product of connected and simply connected simple algebraic groups, its (prounipotent part of the) Iwahori subgroup (we take the product over simple factors) defines a subgroup scheme $\mathbf{R}_{\mathbb{Z}}^{0} \subset \mathbf{R}_{\mathbb{Z}}$ such that

$$
\mathbf{R}_{\mathbb{Z}}^{0} \cap \mathbf{T}_{\mathbb{Z}}=\operatorname{Spec} \mathbb{Z}
$$

(scheme-theoretically). Since $[L(J), L(J)] \subset G$ defines a closed group subscheme over $\mathbb{Z}$ (thanks to the fact that the corresponding modified enveloping algebras share the same $\mathbb{Z}$-basis, by [41, 43, 67]), we deduce that the map $\mathbb{Z}\left[\mathbf{I}_{\mathbb{Z}}^{1}\right] \rightarrow \mathbb{Z}\left[\mathbf{R}_{\mathbb{Z}}^{0}\right]$ must be surjective. The one-parameter subgroups corresponding to the unipotent radical of $P(\mathrm{~J})$ generate a subgroup $\mathbf{R}_{\mathbb{Z}}^{+} \subset \mathbf{R}_{\mathbb{Z}} \subset \mathbf{I}_{\mathbb{Z}}^{1}$ such that $\mathbb{Z}\left[\mathbf{I}_{\mathbb{Z}}^{1}\right] \rightarrow \mathbb{Z}\left[\mathbf{R}_{\mathbb{Z}}^{+}\right]$is surjective. In view of the $P$-weight decomposition, we deduce that $\mathbf{R}_{\mathbb{Z}}^{0} \times \mathbf{R}_{\mathbb{Z}}^{+} \cong \mathbf{R}_{\mathbb{Z}}$. From equation (4.4), we derive $\mathbb{Z}[\mathbf{L}] \cap \mathbb{Z}[\mathbf{R}]=\mathbb{Z}$. Therefore, we have a surjection

$$
m_{\mathbb{Z}}^{*}: \mathbb{Z}\left[\mathbf{I}^{1}\right] \longrightarrow \mathbb{Z}[\mathbf{L}] \otimes_{\mathbb{Z}} \mathbb{Z}[\mathbf{R}]
$$

The ideal ker $m_{\mathbb{Z}}^{*}$ must be flat over $\mathbb{Z}$, and hence ker $m_{\mathbb{C}}^{*} \neq 0$ if ker $m_{\mathbb{Z}}^{*} \neq 0$. We know that $m_{\mathbb{C}}^{*}$ must be an isomorphism by formula (4.3). This is a contradiction, and hence $m_{\mathbb{Z}}^{*}$ must be an isomorphism (compare [31, Theorem 5.8]).

In view of the flatness of the group schemes over $\mathbb{Z}$ afforded by the foregoing, we conclude that

$$
\mathbf{R}_{\mathbb{K}} \subset \operatorname{Ad}\left(\dot{w} \dot{w}_{0}\right)([P(\mathrm{~J}), P(\mathrm{~J})][[z]]) \cap \mathbf{I}_{\mathbb{K}}^{1}
$$

is in fact an isomorphism as schemes. By construction, $\mathbb{Z}[w]$ is precisely the subring of $\mathbb{Z}\left[\mathbf{I}_{\mathbb{K}}^{1}\right]$ that is invariant under the $\mathbf{R}_{\mathbb{Z}}$-action. It follows that the image of the composition map

$$
\mathbb{Z}[w] \hookrightarrow \mathbb{Z}\left[\mathbf{I}^{1}\right] \stackrel{\cong}{\longrightarrow} \mathbb{Z}[\mathbf{L}] \otimes \mathbb{Z}[\mathbf{R}]
$$

is equal to $\mathbb{Z}[\mathbf{L}] \otimes_{\mathbb{Z}} \mathbb{Z}$. Hence we have

$$
\operatorname{Spec} \mathbb{K}[w]=\mathbf{L}_{\mathbb{K}} \cong \mathbf{I}_{\mathbb{K}} /\left(H_{\mathbb{K}} \cdot \operatorname{Ad}\left(\dot{w} \dot{w}_{0}\right)([P(\mathrm{~J}), P(\mathrm{~J})][[z]]) \cap \mathbf{I}_{\mathbb{K}}\right)
$$

Therefore, each $\mathbf{Q}_{G}(w)_{\mathbb{K}}$ contains Spec $\mathbb{K}[w]$ as a Zariski open I-orbit, and it admits a unique $\left(H \times \mathbb{G}_{m}\right)_{\mathbb{K}}$-fixed point, as required.

Corollary 4.12. The ring $R(\mathrm{~J})_{\mathbb{K}}$ is integral for each $\mathrm{J} \subset \mathrm{I}$.

Remark 4.13. We refer to Proposition A.1 for the general case of Corollary 4.12.

Proof of Corollary 4.12. It suffices to prove the case where $\mathrm{J}=\emptyset$, since a subring of an integral ring is integral. We borrow notation from the proof of Lemma 4.11. We have an inclusion

$$
R_{e}=\bigoplus_{\lambda \in P_{+}} \mathbb{W}(\lambda)_{\mathbb{Z}}^{\vee} \hookrightarrow \mathbb{Z}[e] \otimes \bigoplus_{\lambda \in P_{+}} \mathbb{Z} \mathbf{v}_{w_{0} \lambda}^{\vee}
$$

as the Rees construction of formula (4.1). Taking the tensor product $\mathbb{K} \otimes_{\mathbb{Z}} \bullet$ preserves the inclusion in view of the definition of $\mathbb{Z}[e]$. Hence, $\left(R_{e}\right)_{\mathbb{K}}$ is integral as a subring of an integral ring.

Proposition 4.14. Set $\mathrm{J} \subset \mathrm{I}$. The ind-scheme $\left(\mathbf{Q}_{G, \mathrm{~J}}^{\mathrm{rat}}\right)_{\mathbb{K}}$ admits a $G((z))$-action. We have a subset $\mathbf{Q}_{\mathbb{K}}^{\prime}$ of the set of $\mathbb{K}$-valued points of $\left(\mathbf{Q}_{G, \mathrm{~J}}^{\mathrm{rat}}\right)_{\mathbb{K}}$ that is in bijection with

$$
G((z)) /(H(\mathbb{K}) \cdot[P(\mathrm{~J}), P(\mathrm{~J})]((z))) .
$$

Proof. In view of Lemma 4.11, the proof for general $\mathrm{J}$ is completely parallel to the case of $\mathrm{J}=\emptyset$. Hence we concentrate in this proof on the case where $J=\emptyset$. 
Set $w \in W_{\text {af }}$ and $\beta \in Q^{\vee}$. We have an isomorphism $R_{w} \cong R_{w t_{\beta}}$ as rings with $U_{\mathbb{Z}}^{+}$-action, by Corollary 3.28 .

In particular, we have an isomorphism $\mathbf{Q}_{G}(w)_{\mathbb{K}} \cong \mathbf{Q}_{G}\left(w t_{\beta}\right)_{\mathbb{K}}$ of schemes with $\mathbf{I}$-actions for each $\beta \in Q^{\vee}$. This implies that $\mathbf{Q}_{G}\left(w t_{\beta}\right)_{\mathbb{K}}$ has a Zariski open subset of the shape

$$
\mathbf{I} /\left(H \cdot\left(\operatorname{Ad}\left(\dot{w} \dot{t}_{\beta} \dot{w}_{0}\right)(N((z))) \cap \mathbf{I}\right)\right) \cong \mathbf{I} /\left(H \cdot\left(\operatorname{Ad}\left(\dot{w} \dot{w}_{0}\right)(N((z))) \cap \mathbf{I}\right)\right),
$$

where we used the fact that $\operatorname{Ad}\left(\dot{w}_{0}\right)(N((z)))$ is invariant under the $\operatorname{Ad}\left(\dot{t}_{\beta}\right)$-action.

The ring $\left(R_{w}\right)_{\mathbb{K}}$ admits an action of $S L(2, i)$ whenever $s_{i} w \leq_{\frac{\infty}{2}} w\left(i \in \mathrm{I}_{\mathrm{af}}\right)$, since each $\mathbb{W}_{w w_{0}}(\lambda)_{\mathbb{K}}$ $\left(\lambda \in P_{+}\right)$admits an action of $S L(2, i)$ by Corollary 3.16. Hence, $\operatorname{Proj}\left(R_{w}\right)_{\mathbb{K}}$ admits an action of $S L(2, i)$ if $s_{i} w \leq_{\frac{\infty}{2}} w$. In particular, the proalgebraic group $\mathbf{I}(i)$ acts on $\operatorname{Proj}\left(R_{w}\right)_{\mathbb{K}}$ if $s_{i} w \leq_{\frac{\infty}{2}} w$. Hence the ind-limit

$$
\left(\mathbf{Q}_{G}^{\mathrm{rat}}\right)_{\mathbb{K}}=\bigcup_{w \in W_{\mathrm{af}}} \mathbf{Q}_{G}(w)_{\mathbb{K}}=\underset{w}{\lim } \operatorname{Proj}\left(R_{w}\right)_{\mathbb{K}}
$$

admits an action of $\mathbf{I}(i)$ for each $i \in \mathrm{I}_{\mathrm{af}}$ (which coincide on $\mathbf{I}$ ). By rank 2 calculations, they induce an action of $N_{G((z))}(H(\mathbb{K}))$ on $\left(\mathbf{Q}_{G}^{\text {rat }}\right)_{\mathbb{K}}$. The intersections of $N_{G((z))}(H(\mathbb{K}))$ and $\mathbf{I}(\mathbb{K})$ or $\mathbf{I}(i)(\mathbb{K})\left(i \in \mathrm{I}_{\mathrm{af}}\right)$ inside $G((z))$ define common actions on $\left(\mathbf{Q}_{G}^{\text {rat }}\right)_{\mathbb{K}}$. The system of groups $\left(\mathbf{I}(\mathbb{K}), N_{G((z))}(H(\mathbb{K})), \mathbf{I}(i)(\mathbb{K}) ; i \in \mathrm{I}_{\mathrm{af}}\right)$ (in the sense of [57, Definition 5.1.6]) admits a map from the system of groups in $[57, \S 6.1 .16]$ for $\widetilde{\mathfrak{g}}$ (with exponential maps replaced by one-parameter subgroups). Hence, [57, Theorem 6.1.17] asserts that $G((z))$ acts on $\left(\mathbf{Q}_{G}^{\text {rat }}\right)_{\mathbb{K}}$.

The Bruhat decomposition of $S L(2, i)$ asserts that $O(w)_{\mathbb{K}} \sqcup \mathbb{O}\left(s_{i} w\right)_{\mathbb{K}}$ admits an $S L(2, i)$-action. This induces an action of $S L(2, i)(\mathbb{K})\left(i \in \mathrm{I}_{\mathrm{af}}\right)$ on the union

$$
\mathbf{Q}_{\mathbb{K}}^{\prime}:=\bigsqcup_{w \in W_{\mathrm{af}}} \mathbb{O}(w)_{\mathbb{K}}(\mathbb{K}) \subset \mathbf{Q}_{G}^{\mathrm{rat}}(\mathbb{K})
$$

Taking into account the fact that each $\mathbb{O}(w)_{\mathbb{K}}$ admits an I-action (Lemma 4.11), we conclude that $\mathbf{Q}_{\mathbb{K}}^{\prime}$ admits an action of $G((z))$ thanks to the Iwasawa decomposition (compare [38, Theorem 2.5])

$$
G((z))=\bigsqcup_{w \in W_{\mathrm{af}}} \mathbf{I}(\mathbb{K}) \dot{w} \dot{w}_{0} H(\mathbb{K}) \cdot N((z)),
$$

as required.

For $\lambda, \mu \in P_{+}$, we have a unique injective $\dot{U}_{\mathbb{Z}}^{0}$-module map

$$
V(\lambda+\mu)_{\mathbb{Z}} \longrightarrow V(\lambda)_{\mathbb{Z}} \otimes_{\mathbb{Z}} V(\mu)_{\mathbb{Z}}
$$

obtained by sending $\mathbf{v}_{\lambda+\mu}^{0}$ to $\mathbf{v}_{\lambda}^{0} \otimes \mathbf{v}_{\mu}^{0}$, which is in fact a $\mathbb{Z}$-direct summand (Corollary 3.12). By extending the scalar, we obtain a unique injective $\left(\dot{U}_{\mathbb{K}}^{0}, \mathbb{K}[[z]]\right)$-bimodule map

$$
\eta_{\lambda, \mu}: V(\lambda+\mu)_{\mathbb{K}} \otimes \mathbb{K}[[z]]\left(V(\lambda)_{\mathbb{K}} \otimes \mathbb{K}[[z]) \otimes_{\mathbb{K}[[z]]}\left(V(\mu)_{\mathbb{K}} \otimes \mathbb{K}[[z]]\right)\right.
$$

Lemma 4.15. For each $\lambda, \mu, \gamma \in P_{+}$, we have

$$
\eta_{\lambda+\mu, \gamma} \circ\left(\mathrm{id} \otimes \eta_{\lambda, \mu}\right)=\eta_{\lambda, \mu+\gamma} \circ\left(\mathrm{id} \otimes \eta_{\mu, \gamma}\right) .
$$

Proof. This is straightforward from the construction (compare Lemma 3.27). 
Proposition 4.16. Assume that char $\mathbb{K} \neq 2$. For each $w \in W_{\mathrm{af}}$ and $\mathrm{J} \subset \mathrm{I}$, we have an $\mathbf{I}$-equivariant rational map

$$
\psi_{w}: \mathbf{Q}_{G, J}(w)_{\mathbb{K}} \rightarrow \bigcup_{m \in \mathbb{Z}} \prod_{i \in \mathbf{I} \backslash J} \mathbb{P}_{\mathbb{K}}\left(V\left(\varpi_{i}\right)_{\mathbb{K}} \otimes z^{m} \mathbb{K}[[z]]\right)=\prod_{i \in \mathbb{I} \backslash J} \mathbb{P}_{\mathbb{K}}\left(V\left(\varpi_{i}\right)_{\mathbb{K}} \otimes \mathbb{K}((z))\right),
$$

which gives rise to a $G((z))$-equivariant rational map

$$
\psi:\left(\mathbf{Q}_{G, J}^{\mathrm{rat}}\right)_{\mathbb{K}} \rightarrow \prod_{i \in \mathbb{I} \backslash \mathrm{J}} \mathbb{P}_{\mathbb{K}}\left(V\left(\varpi_{i}\right)_{\mathbb{K}} \otimes \mathbb{K}((z))\right) .
$$

In addition, the set $(\operatorname{Im} \psi)(\mathbb{K})$ defines a closed (ind-)subscheme of $\prod_{i \in \mathbb{I} \backslash \mathrm{J}} \mathbb{P}_{\mathbb{K}}\left(V\left(\varpi_{i}\right)_{\mathbb{K}} \otimes \mathbb{K}((z))\right)$.

Proof. We have a surjective map

$$
\mathbb{W}\left(\varpi_{i}\right)_{\mathbb{K}} \rightarrow V\left(\varpi_{i}\right)_{\mathbb{K}} \otimes \mathbb{K}[z]
$$

as $\dot{U}_{\mathbb{K}}^{\geq 0}$-modules, since we have the corresponding map over $\dot{U}_{\mathbb{Z}}^{\geq 0}$ such that the $P$-weight $\varpi_{i}$-part is the same (and $V\left(\varpi_{i}\right)_{\mathbb{K}}$ is cyclic as a $\dot{U}_{\mathbb{K}}^{0}$-module). The identification of the $P$-weight $\varpi_{i}$-part also implies that this map commutes with the action of $\tau_{\beta}\left(\beta \in Q^{\vee}\right)$ and extends to a surjective map

$$
\mathbb{X}\left(\varpi_{i}\right)_{\mathbb{K}} \rightarrow V\left(\varpi_{i}\right)_{\mathbb{K}} \otimes \mathbb{K}\left[z, z^{-1}\right]
$$

of $\dot{U}_{\mathbb{K}}$-modules.

This gives a rational map

$$
\mathbb{P}\left(\mathbb{W}\left(\varpi_{i}\right)_{\mathbb{K}}\right) \rightarrow \mathbb{P}\left(V\left(\varpi_{i}\right)_{\mathbb{K}} \otimes \mathbb{K}[z]\right)
$$

and its graded completion

$$
\mathbb{P}\left(\mathbb{W}\left(\varpi_{i}\right)_{\mathbb{K}}^{\wedge}\right) \rightarrow \mathbb{P}\left(V\left(\varpi_{i}\right)_{\mathbb{K}} \otimes \mathbb{K}[[z]]\right)
$$

Taking Corollary 3.26 into account, we have an embedding

$$
\mathbf{Q}_{G, \mathrm{~J}}(e)_{\mathbb{K}} \hookrightarrow \prod_{i \in \mathbb{I} \backslash \mathrm{J}} \mathbb{P}\left(\mathbb{W}\left(\varpi_{i}\right)_{\mathbb{K}}^{\wedge}\right)
$$

This yields a rational map

$$
\psi_{e}: \mathbf{Q}_{G, J}(e)_{\mathbb{K}} \rightarrow \prod_{i \in \mathbb{I} \backslash \mathrm{J}} \mathbb{P}\left(V\left(\varpi_{i}\right)_{\mathbb{K}} \otimes \mathbb{K}[[z]]\right)
$$

as a composition. This map is $G[[z]]$-equivariant by construction.

For each $w \in W$, we can choose $\beta \in Q_{+}^{\vee}$ such that $\mathbf{Q}_{G, \mathrm{~J}}(w)_{\mathbb{K}} \cong \mathbf{Q}_{G, \mathrm{~J}}\left(w t_{\beta}\right)_{\mathbb{K}} \subset \mathbf{Q}_{G, \mathrm{~J}}(e)_{\mathbb{K}}$, by Corollary 3.28 and Lemma 3.29. Hence we obtain the map $\psi_{w}$ for every $w \in W_{\text {af }}$ as the composition of the foregoing maps (up to grading shifts). We fix the effect of grading shifts from $\psi_{w}$ to $\psi_{w t_{\beta}}$ by applying $\tau_{w_{0} \beta}$ on $\mathbb{W}\left(\varpi_{i}\right)_{\mathbb{K}}^{\wedge}$ for each $i \in \mathrm{I} \backslash \mathrm{J}$ in formula (4.6). Then the $\tau_{w_{0} \beta}$-action (on the completions of $\left.\left\{\mathbb{X}\left(\varpi_{i}\right)_{\mathbb{K}}\right\}_{i \in \mathrm{I} \backslash \mathrm{J}}\right)$ transfers $\psi_{w}$ to $\psi_{w t_{\beta}}$ for each $\beta \in Q^{\vee}$, and is compatible with the restrictions to $\mathbf{Q}_{G}(\bullet)$ s by Lemma 3.17(1). Hence, we obtain the map $\psi$ of ind-schemes. This map is $G((z))$-equivariant in our sense. This proves the first assertion.

From now on, we concentrate on the second assertion. 
Each $\left(H \times \mathbb{G}_{m}\right)$-fixed point of $\mathbb{O}(w)_{\mathbb{K}}\left(w \in W_{\text {af }}\right)$ is contained in the domain of $\psi$, and their images are distinct by inspection. It follows that $\mathbf{Q}_{\mathbb{K}}^{\prime}$ is contained in the domain of $\psi$, and the restriction of $\psi$ to $\mathbf{Q}_{\mathbb{K}}^{\prime}$ is injective by examining the stabiliser of the $\mathbf{I}(\mathbb{K})$-actions at each $\left(H \times \mathbb{G}_{m}\right)$-fixed point.

In view of Theorem 3.18, the maps $\left\{\eta_{\lambda, \mu}\right\}_{\lambda, \mu}\left(\lambda, \mu \in P_{\mathrm{J},+}\right)$ induce a commutative diagram of $\dot{U}_{\mathbb{K}}^{\geq 0}-$ modules:

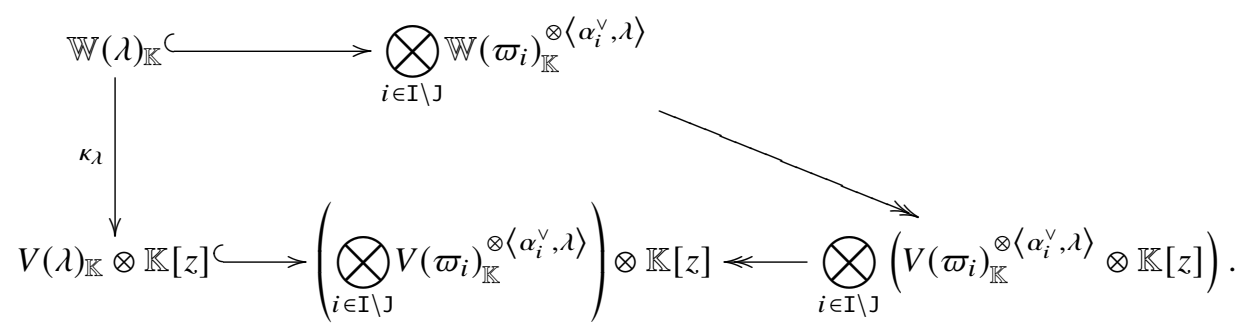

Here the map $\kappa_{\lambda}$ is well defined by examination of the degree 0 part and the action of $E_{0}=F_{\vartheta} \otimes z$ (where $F_{\vartheta}$ is a nonzero vector in the $-\vartheta$-weight space of $\boldsymbol{n}^{-}$). This commutative diagram also commutes with the translation by $\tau_{\beta}\left(\beta \in Q^{\vee}\right)$ by construction. Moreover, we have $\kappa_{\lambda}\left(\mathbf{v}_{w \lambda}\right) \neq 0$ for each $w \in W_{\text {af }}$. Therefore, the map $\kappa_{\lambda}$ must be surjective whenever its $d$-degree belongs to $\sum_{i \in \mathrm{I} \backslash \mathrm{J}} \mathbb{Z}\left\langle\alpha_{i}^{\vee}, \lambda\right\rangle$.

For each $i \in \mathrm{I} \backslash J$, the $\mathbb{Z}\left[\frac{1}{2}\right]$-integral structure of $V\left(2 \varpi_{i}\right) \otimes_{\mathbb{C}} \mathbb{C}\left[z, z^{-1}\right]$ at the odd degree and even

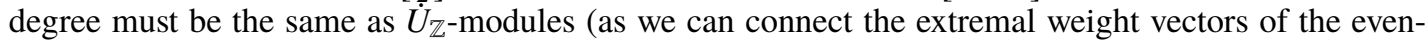
degree and odd-degree parts using the $\mathfrak{s l}(2)$-strings of length 3). Therefore, for $\lambda=\varpi_{i}, \varpi_{i}+\varpi_{j}, 2 \varpi_{i}$ $(i, j \in \mathrm{I} \backslash \mathrm{J})$, the map $\kappa_{\lambda}$ is surjective.

Consider a (representative of the) image

$$
\psi(x)=\left(x_{i}\right) \in \prod_{i \in \mathrm{I} \backslash \mathrm{J}} V\left(\varpi_{i}\right)_{\mathbb{K}} \otimes \mathbb{K}((z))
$$

of a $\mathbb{K}$-valued point $x \in \mathbf{Q}_{G, \mathrm{~J}}(e)_{\mathbb{K}}$ under $\psi$. We consider its lifts $\widetilde{x}_{i} \in \mathbb{W}\left(\varpi_{i}\right)_{\mathbb{K}}^{\wedge}$ and $\widetilde{x}_{j} \in \mathbb{W}\left(\varpi_{j}\right)_{\mathbb{K}}^{\wedge}$. They must belong to

$$
\operatorname{Im}\left(\mathbb{W}\left(\varpi_{i}+\varpi_{j}\right)_{\mathbb{K}}^{\wedge} \longrightarrow\left(\mathbb{W}\left(\varpi_{i}\right)_{\mathbb{K}} \otimes \mathbb{W}\left(\varpi_{j}\right)_{\mathbb{K}}\right)^{\wedge}\right)
$$

in order to satisfy the defining relations of $R_{\mathbb{K}}$. In view of the commutative diagram (4.7) for $\lambda=\varpi_{i}+\varpi_{j}$, we deduce an equation

$$
x_{i} \otimes_{\mathbb{K}[[z]]} x_{j} \in \operatorname{Im} \eta_{\varpi_{i}, \varpi_{j}}, \quad i, j \in \mathrm{I} \backslash J
$$

Since the relation of the ring $\bigoplus_{\lambda \in P_{\mathrm{J}_{+}}} V(\lambda)_{\mathbb{K}}^{*}$ is generated by $P$-degrees $2 \varpi_{i}$ and $\varpi_{i}+\varpi_{j}$ for $i, j \in \mathrm{I}$ ([14, Theorem 3.5.3]), this defines an element of

$$
G((z)) /[P(\mathrm{~J}), P(\mathrm{~J})]((z)) \subset \overline{G /[P(\mathrm{~J}), P(\mathrm{~J})]}(\mathbb{K}((z)))
$$

through quadratic relations $[29, \S 4]$, where

$$
\overline{G /[P(\mathrm{~J}), P(\mathrm{~J})]}=\operatorname{Spec} \mathbb{K}[G /[P(\mathrm{~J}), P(\mathrm{~J})]]
$$

is the basic affine space. Therefore, applying some $\tau_{\beta}\left(\beta \in Q^{\vee}\right)$ if necessary, we conclude that if a $\mathbb{K}$ valued point $x$ of $\mathbf{Q}_{G, \text { rat }}^{\text {rat }}$ belongs to the domain of $\psi$, then $\psi(x)$ belongs to the set of $\mathbb{K}$-valued points of the image given by formula (4.9). Taking Proposition 4.14 (and its $G((z))$-action) and formula (4.8) into account, we conclude the second assertion. 
Corollary 4.17. Keep the setting of Proposition 4.16. The map $\psi$ induces a bijection of $\mathbb{K}$-valued points between the domain and the range.

Proof. By abuse of convention, we regard $\mathbf{Q}_{\mathbb{K}}^{\prime}$ as subsets of both $\mathbf{Q}_{G}^{\text {rat }}(\mathbb{K})$ and $(\operatorname{Im} \psi)(\mathbb{K})$. Let $x^{\prime} \in$ $\mathbf{Q}_{G}^{\text {rat }}(\mathbb{K})$ be a preimage of an $\left(H \times \mathbb{G}_{m}\right)(\mathbb{K})$-fixed point $x \in \mathbf{Q}_{\mathbb{K}}^{\prime}$ through $\psi$. Consider the embedding

$$
\left(\mathbf{Q}_{G, \mathrm{~J}}^{\mathrm{rat}}\right)_{\mathbb{K}} \hookrightarrow \prod_{i \in \mathrm{I} \backslash \mathrm{J}} \mathbb{P}\left(\mathbb{X}\left(\varpi_{i}\right)_{\mathbb{K}}^{\wedge}\right)
$$

that prolongs formula (4.6). Let $x^{\prime}=\left(x_{i}^{\prime}\right)_{i \in \mathrm{I} \backslash \mathrm{J}}\left(\operatorname{resp} ., x=\left(x_{i}\right)_{i \in \mathrm{I} \backslash \mathrm{J}}\right.$ ) be the coordinate of $x^{\prime}$ (resp., $x$ ) through this embedding. We can regard $x_{i}^{\prime} \in \mathbb{X}\left(\varpi_{i}\right)_{\mathbb{K}}^{\wedge}$, which admits a decomposition

$$
x_{i}^{\prime}=\prod_{\mu} x_{i}^{\prime}[\mu]
$$

where $\mu \in P$ runs over the $P$-weights of $\mathbb{X}\left(\varpi_{i}\right)_{\mathbb{K}}^{\wedge}\left(\right.$ or $\left.\mathbb{W}\left(\varpi_{i}\right)_{\mathbb{K}}^{\wedge}\right)$. Let $d_{i}^{\prime}[\mu]$ be the degree of the lowest $d$-degree nonzero contribution of $x_{i}^{\prime}[\mu]$ (or $\infty$ if $x_{i}^{\prime}[\mu]=0$ ) for each $i \in \mathrm{I} \backslash \mathrm{J}$ and $\mu \in P$. Let $d_{i}$ be the $d$-degree of $x_{i}$ for each $i \in \mathrm{I} \backslash \mathrm{J}$ (remember that $x$ is $\left(H \times \mathbb{G}_{m}\right)(\mathbb{K})$-fixed). Note that we have $x_{i}\left[u \varpi_{i}\right]=x_{i}^{\prime}\left[u \varpi_{i}\right]$ for every $i \in \mathrm{I}$ and $u \in W$, since

$$
\mathbb{W}\left(\varpi_{i}\right)_{\mathbb{K}} \text { and } V\left(\varpi_{i}\right)_{\mathbb{K}} \otimes \mathbb{K}[z] \text { share the same } P \text {-weight } u \varpi_{i} \text {-parts. }
$$

For each $\gamma \in Q^{\vee}$, we have a collection of the automorphisms of the vector spaces $\left\{\mathbb{X}(\lambda)_{\mathbb{K}}\right\}_{\lambda \in P_{+}}$by shifting the $\left(d\right.$-)gradings of the weight $\mu$ parts by $\langle\gamma, \mu\rangle(\mu \in P)$. This defines an automorphism of $R_{\mathbb{K}}^{+}$, and hence defines an automorphism of $\mathbf{Q}_{G}^{\text {rat }}(\mathbb{K})$. By using this twist for an appropriate $\gamma \in Q^{\vee}$, we can assume that

$$
d_{i}^{\prime}[\mu] \gg d_{i}^{\prime}\left[u \varpi_{i}\right]=d_{i}, \quad \mu \neq u \varpi_{i},
$$

for each $i \in \mathrm{I} \backslash \mathrm{J}$ and fixed $u \in W$, from the fact that every $P$-weight of $W\left(\varpi_{i}\right)_{\mathbb{K}}$ is $\leq \varpi_{i}$. We have $x_{i} \in \mathbb{W}_{w w_{0}}\left(\varpi_{i}\right)_{\mathbb{K}}$ for some $w \in W_{\text {af }}$ and all $i \in \mathrm{I} \backslash J$. Formula (4.11) implies $x_{i}^{\prime} \in \mathbb{W}_{w w_{0}}\left(\varpi_{i}\right)_{\mathbb{K}}^{\wedge}$ for all $i \in \mathrm{I} \backslash \mathrm{J}$ ([44, Theorem 5.17]). Hence, we have $x^{\prime} \in \mathbf{Q}_{G, \mathrm{~J}}(w)_{\mathbb{K}}$ for $w \in W_{\text {af }}$ such that $x$ is the unique $\left(H \times \mathbb{G}_{m}\right)$-fixed point of $\mathbb{O}(\mathrm{J}, w)_{\mathbb{K}}$.

From fact (4.10), the $\left(H \times \mathbb{G}_{m}\right)$-action shrinks $x^{\prime}$ to $x$. In view of its proof, the Zariski open set $\mathbb{O}(\mathrm{J}, w)_{\mathbb{R}}$ in Lemma 4.11 is obtained by localising along the vectors $\left\{\mathbf{v}_{w w_{0} \lambda}^{\vee}\right\}_{\lambda \in P_{\mathrm{J},+}}$, which pairs nontrivially with $x^{\prime}$. Therefore, we necessarily have $x^{\prime} \in \mathbb{O}(\mathrm{J}, w)_{\mathbb{K}}(\mathbb{K})$. As $\mathbb{O}(\mathrm{J}, w)_{\mathbb{K}}(\mathbb{K}) \subset \mathbf{Q}_{\mathbb{K}}^{\prime}$ by Proposition 4.14 and its proof, we deduce $x=x^{\prime}$. Since $\mathbf{Q}_{\mathbb{K}}^{\prime}$ is stable under the action of $Q^{\vee}$, as well as $G((z))$, we conclude that $x=x^{\prime}$ holds for a preimage of $x^{\prime}$ of every $x \in \mathbf{Q}_{\mathbb{K}}^{\prime}$, as required.

Theorem 4.18. Assume that char $\mathbb{K} \neq 2$. For each $\mathrm{J} \subset \mathrm{I}$, we have a closed immersion of ind-schemes

$$
\left(\mathbf{Q}_{G, \mathrm{~J}}^{\mathrm{rat}}\right)_{\mathbb{K}} \longrightarrow \prod_{i \in \mathrm{I} \backslash J} \mathbb{P}\left(V\left(\varpi_{i}\right)_{\mathbb{K}} \otimes \mathbb{K}((z))\right) .
$$

In particular, the set of $\mathbb{K}$-valued points of the ind-scheme $\left(\mathbf{Q}_{G, \mathrm{~J}}^{\mathrm{rat}}\right)_{\mathbb{K}}$ is in bijection with

$$
G((z)) /(H(\mathbb{K}) \cdot[P(\mathrm{~J}), P(\mathrm{~J})]((z))) .
$$

Proof. By construction, the locus $E \subset \mathbf{Q}_{G, \text { J }}^{\text {rat }}$ on which $\psi$ (borrowed from Proposition 4.16) is not defined is an ind-subscheme. The map $\psi$ is $G((z))$-equivariant. It follows that $E$ admits a $G((z))$-action as ind-schemes. The map

$$
\mathbf{Q}_{G, J}(e)_{\mathbb{K}} \longrightarrow \mathbb{P}\left(\mathbb{W}\left(\varpi_{i}\right)_{\mathbb{K}}^{\wedge}\right)
$$


(borrowed from the proof of Proposition 4.16) sends an irreducible component of $\left(E \cap \mathbf{Q}_{G, \mathrm{~J}}(e)_{\mathbb{K}}\right)$ onto a closed subscheme of $\mathbb{P}\left(\operatorname{ker}\left(\mathbb{W}\left(\varpi_{i}\right)_{\mathbb{K}}^{\wedge} \rightarrow V\left(\varpi_{i}\right)_{\mathbb{K}} \otimes \mathbb{K}[[z]]\right)\right)$ for some $i \in I \backslash J$. In particular, $\left(E \cap \mathbf{Q}_{G, J}(e)_{\mathbb{K}}\right) \subset \mathbf{Q}_{G, \mathrm{~J}}(e)_{\mathbb{R}}$ is a closed subscheme. Taking into account the $\left\{\tau_{\beta}\right\}_{\beta}$-actions (Lemma 3.17), we have $E \neq \emptyset$ if and only if $\left(E \cap \mathbf{Q}_{G, \mathrm{~J}}(e)_{\mathbb{K}}\right) \neq \emptyset$, and $\psi$ is a closed immersion if and only if $\psi_{e}$ is a closed immersion (compare Lemma 3.29).

The one-parameter subgroup $a=(\xi, 1): \mathbb{G}_{m} \rightarrow\left(H \times \mathbb{G}_{m}\right)$ for $\xi \in Q_{<}^{\vee}$ attracts every point of $\mathbb{P}\left(\mathbb{W}\left(\varpi_{i}\right)_{\mathbb{K}}\right)$ into its $\left(H \times \mathbb{G}_{m}\right)$-fixed points (by setting $\left.t \rightarrow 0\right)$. In particular, $E$ has an $\left(H \times \mathbb{G}_{m}\right)$-fixed point that is not realised by $\mathbf{Q}_{\mathbb{K}}^{\prime}$. Since the $\left(H \times \mathbb{G}_{m}\right)$-fixed point of bounded $d$-degree is captured by the corresponding degree terms of $R_{\mathbb{K}}$, it follows that the ind-scheme $E$ intersects with $\mathbb{Q}_{j}^{\prime}(v, e)$ for some $v \in W_{\text {af }}$. The intersection of $\mathscr{Q}_{j}^{\prime}(v, e)_{\mathbb{K}}$ and $\mathbf{Q}_{\mathbb{K}}^{\prime}$ (as the set of $\mathbb{K}$-valued points) defines a closed subset $Y$ of (a product of) a finite-dimensional projective space, by Proposition 4.16 and Corollary 4.17. In particular, $Y$ acquires the structure of a proper scheme through $\psi$. From this viewpoint, Corollary 4.17 provides a $\psi$-section of $Y$ that defines a bijection of $\mathbb{K}$-valued points with a Zariski open subset $Q_{j}^{\prime}(v, e)_{\mathbb{K}} \backslash E$ of $Q_{j}^{\prime}(v, e)_{\mathbb{K}}$. Since $\mathbf{Q}_{\mathbb{K}}^{\prime}$ admits a homogeneous $G((z))$-action, we can think of $\psi$ as an everywhere-defined section of a vector bundle over the image (whose fibre is a product of $\left.\operatorname{ker}\left(\mathbb{W}\left(\varpi_{i}\right)_{\mathbb{K}}^{\wedge} \rightarrow V\left(\varpi_{i}\right)_{\mathbb{K}} \otimes \mathbb{K}[[z]]\right) s\right)$. This implies that $Q_{\jmath}^{\prime}(v, e)_{\mathbb{K}} \backslash E$ can be seen as an everywheredefined section of a vector bundle over the image (whose fibre is a product of finite-dimensional subspaces of $\left.\operatorname{ker}\left(\mathbb{W}\left(\varpi_{i}\right)_{\mathbb{K}} \rightarrow V\left(\varpi_{i}\right)_{\mathbb{K}} \otimes \mathbb{K}[z]\right) s\right)$. Since $Q_{\jmath}^{\prime}(v, e)_{\mathbb{K}}$ is a finite-type scheme defined over an algebraically closed field $\mathbb{K}$, it has a dense subset formed by its $\mathbb{K}$-valued points ([36, Corollarie 10.4.8]). Thus, the section $\psi$ can be seen as that of schemes. Therefore, $\mathscr{Q}_{\jmath}^{\prime}(v, e)_{\mathbb{R}} \backslash E$ is proper by itself. In conclusion, $Q_{j}^{\prime}(v, e)_{\mathbb{K}} \backslash E$ can be seen as a connected component of $Q_{\mathrm{J}}^{\prime}(v, e)_{\mathbb{K}}$ (as being an open and closed subset) which does not intersect with $E$. It follows that if $E \neq \emptyset$ as a scheme, then the scheme $Q_{j}^{\prime}(v, e)$ must have at least two connected components. Therefore, the projective coordinate ring $R_{e}^{v}(\mathrm{~J})_{\mathbb{K}}$ of $Q_{\jmath}^{\prime}(v, e)$ must be nonintegral. The same is true if we replace $v$ with a smaller element with respect to $<\frac{\infty}{2}$.

Hence, we can find $f, g \in R_{e}^{v}(J)_{\mathbb{K}} \backslash\{0\}$ such that $f g=0$ for $v \ll \frac{\infty}{2} e$. Since $E$ and its complement are $\mathbb{G}_{m}$-stable, we can assume that $f$ and $g$ are $\mathbb{G}_{m}$-eigenfunctions. Since $E$ defines a connected component of $Q_{\jmath}^{\prime}(v, e)$ for every $v \ll \frac{\infty}{2} e$, we can fix the degrees of $f$ and $g$ for every $v \ll \frac{\infty}{2} e$. For a fixed degree, the $d$ graded component of $R_{e}^{v}(\mathrm{~J})_{\mathbb{K}}$ and $R_{e}(\mathrm{~J})_{\mathbb{K}}$ are in common for $v \ll \frac{\infty}{2} e$. Since $R_{e}^{v}(\mathrm{~J})_{\mathbb{K}}$ is a quotient ring of $R_{e}(\mathrm{~J})_{\mathbb{K}}$, we can find $\mathbb{G}_{m}$-eigenfunctions $f, g \in R_{e}(\mathrm{~J})_{\mathbb{K}} \backslash\{0\}$ such that $f g=0$. This implies that the ring $R_{\mathbb{K}}$ is also nonintegral. This contradicts Corollary 4.12, and hence we deduce $E=\emptyset$ as an ind-scheme. Therefore, we conclude that $\psi$ is in fact a genuine morphism (instead of a rational map) of ind-schemes.

Next we prove that $\psi$ (or rather $\psi_{e}$ ) defines a closed immersion. By Lemma 4.11 and the fact that $\psi$ is a morphism, we deduce that $\psi$ induces an isomorphism between the function fields of $\mathbf{Q}_{G, \mathrm{~J}}(e)_{\mathbb{K}}$ and its image under $\psi$. An irreducible component $Z$ of a reduced scheme $Q_{j}^{\prime}(v, e)_{\mathbb{R}}$ shares a Zariski dense subset with a unique orbit $\mathbb{O}(\mathrm{J}, w)_{\mathbb{K}}\left(w \in W_{\mathrm{af}}\right)$. Hence the variety $\left(Z \cap \mathbb{O}(\mathrm{J}, w)_{\mathbb{K}}\right)$ and its image under $\psi$ are presented by the quotients of a common polynomial ring $\mathbb{K}\left[O(J, w)_{\mathbb{K}}\right]$ of infinite variables. In view of the $d$-degree bounds from above and below offered by $\mathbb{W}_{v w_{0}}^{-}\left(\varpi_{i}\right)_{\mathbb{K}}$ and $\mathbb{W}_{w_{0}}\left(\varpi_{i}\right)_{\mathbb{K}}$ for each $i \in \mathrm{I} \backslash \mathrm{J}$, these two quotients of $\mathbb{K}\left[O(J, w)_{\mathbb{K}}\right]$ factor through a common polynomial ring of finite variables. Thus, we deduce that $\psi$ also induces an isomorphism between the function fields of $Z$ and $\psi(Z)$. Once we fix an integer $d_{0}$, two rings $R_{e}^{v}(\mathrm{~J})_{\mathbb{K}}$ and $R_{e}(\mathrm{~J})_{\mathbb{K}}$ share the same $\left(d\right.$-)grading $d_{0}$-part for $v \ll \frac{\infty}{2} e$. Hence, it suffices to prove that $\psi$ restricts to a closed immersion of $Q_{J}^{\prime}(v, e)_{\mathbb{K}}$ for $v \ll \frac{\infty}{2} e$. Here $R_{e}^{v}(\mathrm{~J})_{\mathbb{K}}$ is weakly normal by Lemma 4.7. Let $R^{\prime}$ be the multihomogeneous coordinate ring of the (reduced induced structure of the) closed subscheme of $\prod_{i \in \mathrm{I} \backslash \mathrm{J}} \mathbb{P}\left(V\left(\varpi_{i}\right)_{\mathbb{K}} \otimes \mathbb{K}((z))\right)$ defined by $\psi\left(\mathcal{Q}_{\mathrm{J}}^{\prime}(v, e)_{\mathbb{K}}\right)$. Then if we consider each maximal integral quotient, we deduce that the weak normalisation of $R^{\prime}$ is precisely $R_{e}^{v}(\mathrm{~J})_{\mathbb{K}}$ (up to irrelevant locus). This implies that $\psi\left(Q_{\mathrm{J}}^{\prime}(v, e)_{\mathbb{R}}\right)$ defines a closed subscheme for each $v \in W_{\text {af }}$ as required.

Since $\psi$ is a closed immersion, the rest of the assertion follows from Proposition 4.16. This completes the proof.

Corollary 4.19 (Corollary of the proof of Theorem 4.18). Set $w \in W_{\mathrm{af}}$ and $\mathrm{J} \subset \mathrm{I}$. Every two rational functions on $\mathbf{Q}_{G, \mathrm{~J}}(w)_{\mathbb{K}}$ are distinguished by a pair of $\mathbb{K}$-valued points of $Q_{\mathrm{J}}^{\prime}(v, w)_{\mathbb{K}}$ for some $v \in W_{\mathrm{af}}$. In particular, the union $\bigcup_{v \in W_{\mathrm{af}}} Q_{\mathrm{J}}^{\prime}(v, w)_{\mathbb{R}}$ is Zariski dense in $\mathbf{Q}_{G, \mathrm{~J}}(w)_{\mathbb{K}}$. 
Corollary 4.20. The conclusions of Theorems 2.4 and 2.5 hold when we replace $\mathbb{C}$ with an algebraically closed field $\mathbb{K}$ of characteristic $\neq 2$.

Proof. In view of Theorem 4.18, all the results in Theorems 2.4 and 2.5 are consequences of the corresponding set-theoretic considerations.

Corollary 4.21. We have $Q^{\prime}(v, w)_{\mathbb{K}} \neq \emptyset$ if and only if $v \leq_{\frac{\infty}{2}} w$.

Proof. By Lemma 4.5, it remains to prove that $Q^{\prime}(v, w)_{\mathbb{K}}=\emptyset$ if $\left.v\right\rfloor_{\frac{\infty}{2}} w$. By Theorem 4.18 and the definition of $Q^{\prime}(v, w)_{\mathbb{K}}$, the $\left(H \times \mathbb{G}_{m}\right)$-fixed point of $\mathbb{O}(u)_{\mathbb{K}}$ is contained in $Q^{\prime}(v, w)_{\mathbb{K}}\left(v, w \in W_{\text {af }}\right)$ only if $v \leq_{\frac{\infty}{2}} u \leq_{\frac{\infty}{2}} w$. Since $\leq_{\frac{\infty}{2}}$ is a partial order, the condition $v \Varangle_{\frac{\infty}{2}} w$ implies that $Q^{\prime}(v, w)_{\mathbb{K}}$ has no $\left(H \times \mathbb{G}_{m}\right)$-fixed point. Since $Q^{\prime}(v, w)_{\mathbb{K}}$ is projective by Lemma 4.4 , it carries an $\left(H \times \mathbb{G}_{m}\right)$-fixed point if it is nonempty. Thus, we conclude $\mathbb{Q}^{\prime}(v, w)_{\mathbb{K}}=\emptyset$ as required.

\subsection{Coarse representability of the scheme $\mathrm{Q}_{G}^{\text {rat }}$}

Material in this subsection is rather special in this paper, and is irrelevant to the arguments in the later part such as the normality of quasi-map spaces.

In this subsection, we assume that char $\mathbb{K} \neq 2$, and we drop subscripts $\mathbb{K}$ from $\left(\mathbf{Q}_{G}^{\text {rat }}\right)_{\mathbb{K}}$ and its subschemes to simplify notation.

Let $A f f_{\mathbb{K}}$ be the category of affine schemes over $\mathbb{K}$. We identify $A f f_{\mathbb{K}}^{o p}$ with the category of commutative rings over $\mathbb{K}$. Let $Z a r_{\mathbb{K}}$ denote a big Zariski site over $\mathbb{K}[77$, Section $020 \mathrm{~N}]$. For $X \in Z_{\mathbb{K}}$, the assignment

$$
\operatorname{Zar}_{\mathbb{K}}^{o p} \ni U \mapsto \operatorname{Hom}_{Z a r_{\mathbb{K}}}(U, X) \in \text { Sets }
$$

defines a sheaf $h_{X}$ on $\operatorname{Zar}_{\mathbb{K}}$ [77, Definition 00WR].

For the definition on the coarse moduli functors, we refer to [78, Definition 1.10]. However, we use some modified definitions:

Definition 4.22 (Strict ind-scheme). Let $\mathfrak{X}=\bigcup_{n \geq 0} X_{n}$ be an increasing union of schemes in $Z a r_{\mathbb{K}}$. We call $\left(\mathfrak{X},\left\{X_{n}\right\}_{n}\right)$ (or simply $\left.\mathfrak{X}\right)$ a strict ind-scheme if each inclusion $X_{k} \subset X_{k+1}(k \geq 0)$ is a closed immersion.

Definition 4.23 (Filtered sheaf on $\left.Z a r_{\mathbb{K}}\right)$. A filtered (pre)sheaf $\left(\mathcal{F},\left\{\mathcal{F}_{n}\right\}_{n \geq 0}\right)$ on $\operatorname{Zar}_{\mathbb{K}}$ is a family of (pre)sheaves such that $\mathcal{F}_{k} \subset \mathcal{F}_{k+1}$ for each $k \in \mathbb{Z}_{\geq 0}$ and $\mathcal{F}=\bigcup_{n} \mathcal{F}_{n}$. Let $\left(\mathcal{F},\left\{\mathcal{F}_{n}\right\}_{n \geq 0}\right)$ and $\left(\mathcal{G},\left\{\mathcal{G}_{n}\right\}_{n \geq 0}\right)$ be filtered (pre)sheaves on $\operatorname{Zar}_{\mathbb{K}}$. A morphism $f: \mathcal{F} \rightarrow \mathcal{G}$ of (pre)sheaves is said to be continuous if for each $n \in \mathbb{Z}_{\geq 0}$, there is some $m \in \mathbb{Z}_{\geq 0}$ such that

$$
f\left(\mathcal{F}_{n}\right) \subset \mathcal{G}_{m} \quad \text { and } \quad \mathcal{G}_{n} \cap \operatorname{Im} f \subset f\left(\mathcal{F}_{m}\right)
$$

Let $\left(\mathfrak{F},\left\{F_{n}\right\}_{n}\right)$ be a strict ind-scheme. Then we call $h_{\mathfrak{F}}:=\left(\bigcup_{n} h_{F_{n}},\left\{h_{F_{n}}\right\}_{n \geq 0}\right)$ the filtered sheaf associated to $\mathfrak{F}$.

Definition 4.24 (Coarse ind-representability). Let $\mathcal{X}$ be a filtered (pre)sheaf on $Z a r_{\mathbb{R}}$. Let $\mathfrak{X}$ be a strict ind-scheme over $\mathbb{K}$. We say that $\mathcal{X}$ is coarsely ind-representable by $\mathfrak{X}$ if the following conditions hold:

- We have a continuous morphism $u: \mathcal{X} \rightarrow h_{\mathfrak{X}}$ of filtered (pre)sheaves.

○ $\mathcal{X}(\mathbb{K})=h_{\mathfrak{X}}(\mathbb{k})$ for an overfield $\mathbb{k} \supset \mathbb{K}$. 
○ If $\mathfrak{Y}$ is a strict ind-scheme and we have a continuous morphism $f: \mathcal{X} \rightarrow h_{\mathfrak{Y}}$, it factors as

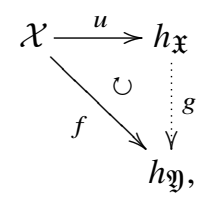

where $g$ is a morphism of presheaves. It is automatic that $g$ is continuous, and hence is induced by a morphism of ind-schemes.

We consider the assignment $\mathcal{Q}$ on $A f f_{\mathbb{K}}^{o p}$ defined as

$$
A f f_{\mathbb{K}}^{o p} \ni R \mapsto \mathcal{Q}(R):=G(R((z))) /(H(R) N(R((z)))) \in \text { Sets. }
$$

For each $n \in \mathbb{Z}_{\geq 0}$, we consider an assignment

$$
A f f_{\mathbb{K}}^{o p} \ni R \mapsto \mathcal{Q}_{n}(R):=\{g \bmod H(R) N(R((z))) \in \mathcal{Q}(R) \mid(\star)\} \in \text { Sets, }
$$

where

$(\star) g \mathbf{v}_{\varpi_{i}}$ has at worst pole of order $n$ on $V\left(\varpi_{i}\right)_{\mathbb{Z}} \otimes_{\mathbb{Z}} R((z))$ for each $i \in \mathrm{I}$.

The assignments $\left(\mathcal{Q},\left\{\mathcal{Q}_{n}\right\}_{n}\right)$ define a filtered presheaf on $Z_{a r_{\mathbb{R}}}$ that we denote by $\mathcal{Q}$.

Lemma 4.25. The ind-scheme $\mathbf{Q}_{G}^{\text {rat }}$ defines a filtered sheaf on $Z a r_{\mathbb{K}}$ given by a strict ind-scheme structure

Proof. A scheme over $\mathbb{K}$ defines a sheaf over $Z a r_{\mathbb{K}}$, and so is its increasing union. In view of Proposition 4.16, the pole order $n$ condition amounts to choosing the I-orbits $\mathbb{O}\left(u t_{\beta}\right)\left(u \in W, \beta \in Q^{\vee}\right)$ such that $\left\langle\beta, \varpi_{i}\right\rangle \geq-n$ (for every $i \in \mathrm{I}$ ), which makes the smaller one a closed subscheme of the larger one (compare Lemma 3.29).

Proposition 4.26. The scheme $\mathbf{Q}_{G}^{\text {rat }}$ coarsely ind-represents the presheaf $\mathcal{Q}$.

Proof. We first construct an injective continuous morphism $\mathcal{Q} \rightarrow h_{\mathbf{Q}_{G}^{\text {rat }}}$ as filtered presheaves on $\operatorname{Zar}_{\mathbb{K}}$ such that $\mathcal{Q}(\mathbb{K})=h_{\mathbf{Q}_{G}^{\text {rat }}}(\mathbb{k})$ for an overfield $\mathbb{k} \supset \mathbb{K}$.

For $R \in A f f_{\mathbb{K}}^{o p}$, the set $\mathcal{Q}(R)$ is represented by a class of $g \in G(R((z)))$ modulo the right action of $H(R) N(R((z)))$. It defines a point of $\mathbf{Q}_{G}^{\text {rat }}(R)$ by applying $g$ on $\left\{\left[\mathbf{v}_{\varpi_{i}}\right]\right\}_{i \in \mathrm{I}} \in \prod_{i \in \mathrm{I}} \mathbb{P}_{R}\left(V\left(\varpi_{i}\right)_{\mathbb{Z}} \otimes_{\mathbb{Z}} R((z))\right)$. Since the $G(R((z)))$-stabiliser of $\mathbf{v}_{\varpi_{i}}$ is precisely $H(R) N(R((z)))$, we conclude an inclusion $\mathcal{Q}(R) \subset$ $h_{\mathbf{Q}_{G}^{\text {rat }}}(R)$. By examining the construction, we deduce that this defines an injective continuous morphism of filtered presheaves.

By the Bruhat decomposition, we have

$$
G(\mathbb{k}((z))) / N(\mathbb{K}((z)))=(G / N)(\mathbb{k}((z)))
$$

for an overfield $\mathbb{k} \supset \mathbb{K}$ (and hence $\mathbb{k}((z))$ is a field). In view of Theorem 4.18, we conclude that $\mathcal{Q} \subset h_{\mathbf{Q}_{G}^{\text {rat }}}$ is an inclusion of filtered presheaves with $\mathcal{Q}(\mathbb{k})=h_{\mathbf{Q}_{G}^{\text {rat }}}(\mathbb{k})$ when $\mathbb{k} \supset \mathbb{K}$ is an overfield.

We verify the versality property. Suppose that we have a strict ind-scheme $\left(\mathfrak{X},\left\{X_{n}\right\}_{n}\right)$ and a continuous morphism $\mathcal{Q} \rightarrow h_{\mathfrak{X}}$. By Lemma 4.11, we deduce that each I-orbit of $\mathbf{Q}_{G}^{\text {rat }}$ defines a subfunctor of $\mathcal{Q}_{n}$ for some $n \geq 0$. The zeroth ind-piece $\left(\mathbf{Q}_{G}^{\text {rat }}\right)_{0}$ in Lemma 4.25 is $\mathbf{Q}_{G}(e)$.

For each $t_{\beta} \leq_{\frac{\infty}{2}} e\left(\beta \in Q_{+}^{\vee}\right)$, we find a reduced expression $t_{\beta}^{-1}=s_{i_{1}} \cdots s_{i_{\ell}}$ (which we record as $\left.\mathbf{i}:=\left(i_{1}, \ldots, i_{\ell}\right)\right)$ and form a scheme

$$
Z(\mathbf{i})^{\circ}:=\mathbf{I}\left(i_{1}\right) \times{ }^{\mathbf{I}} \mathbf{I}\left(i_{2}\right) \times{ }^{\mathbf{I}} \cdots \times \times^{\mathbf{I}} \mathfrak{O}\left(t_{\beta}\right)
$$


and the map

$$
Z(\mathbf{i})^{\circ}=\mathbf{I}\left(i_{1}\right) \times \mathbf{I} \mathbf{I}\left(i_{2}\right) \times^{\mathbf{I}} \cdots \times^{\mathbf{I}} \mathbb{O}\left(t_{\beta}\right) \rightarrow \mathbf{Q}_{G}(e)
$$

(see, e.g., [57, Chapter VIII]; compare [46, §6]). In view of Lemma 4.11, the image of this map contains an open neighbourhood of $\mathbb{O}\left(t_{\beta}\right)$. In view of the $G((z))$-action (or the various $S L(2, i)$-actions for $i \in \mathrm{I}_{\mathrm{af}}$ ) on $\mathbf{Q}_{G}^{\text {rat }}$ and $\mathcal{Q}$, we have a morphism

$$
f_{\mathbf{i}}: h_{Z(\mathbf{i})^{\circ}} \longrightarrow \mathcal{Q}_{0} .
$$

By varying $\mathbf{i}$ (and consequently varying $t_{\beta} \leq_{\frac{\infty}{2}} e$ ), we deduce that the union of the image of the morphisms $\left\{f_{\mathbf{i}}\right\}_{\mathbf{i}}$ exhausts $\mathcal{Q}_{0}(\mathbb{k})$ for an overfield $\mathbb{k} \supset \mathbb{K}$. From the Yoneda embedding, we derive a map

$$
Z(\mathbf{i})^{\circ} \longrightarrow X_{n}
$$

of schemes for some fixed $n \in \mathbb{Z}$. This map factors through a scheme $Z$ that glues (among is) all the closed points that map to the same points in $\mathcal{Q}_{0}$. Such a scheme is integral, as $Z(\mathbf{i})^{\circ} \mathrm{s}$ are integral, and the gluing identifies the Zariski open dense subset $\mathcal{O}(e)$ for distinct is. In addition, we have a birational map $\pi: Z \rightarrow \mathbf{Q}_{G}(e)$, and hence we have

$$
Z(\mathbb{k})=\mathbf{Q}_{G}(e)(\mathbb{k})=\mathcal{Q}_{0}(\mathbb{k}) \text { for an overfield } \mathbb{k} \supset \mathbb{K} .
$$

We prove that $Z=\mathbf{Q}_{G}(e)$ by induction. For each $m \in \mathbb{Z}_{\geq 0}$, let $\mathbf{Q}_{G}(e)_{<m}\left(\operatorname{resp}\right.$., $\left.\mathbf{Q}_{G}(e)_{\leq m}\right)$ be the union of $\mathbf{I}$-orbits in $\mathbf{Q}_{G}(e)$ of the shape $\mathbb{O}(v)$ for $\ell^{\frac{\infty}{2}}(v)<m$ (resp., $\left.\leq m\right)$.

Assume that the map $\pi$ is an isomorphism when restricted to $\mathbf{Q}_{G}(e)_{<m}$; we prove the same is true when restricted to $\mathbf{Q}_{G}(e)_{\leq m}$. The $m=1$ case is afforded by $\mathbb{O}(e) \subset Z$, already used in the foregoing construction.

We have a partial compactification $Z(\mathbf{i})$ of $Z(\mathbf{i})^{\circ}$ with a map $f_{\mathbf{i}}^{+}$:

$$
Z(\mathbf{i}):=\mathbf{I}\left(i_{1}\right) \times \mathbf{I} \mathbf{I}\left(i_{2}\right) \times \mathbf{I} \cdots \times \times^{\mathbf{I}} \mathbf{Q}_{G}\left(t_{\beta}\right) \stackrel{f_{\mathbf{i}}^{+}}{\longrightarrow} \mathbf{Q}_{G}(e) .
$$

Note that we have a surjective morphism induced by $\mathbb{O}\left(t_{\beta}\right) \rightarrow \operatorname{Spec} \mathbb{K}:$

$$
\eta_{\mathbf{i}}: Z(\mathbf{i}) \longrightarrow \mathbf{I}\left(i_{1}\right) \times{ }^{\mathbf{I}} \mathbf{I}\left(i_{2}\right) \times{ }^{\mathbf{I}} \cdots \times{ }^{\mathbf{I}} \operatorname{Spec} \mathbb{K}
$$

where we denote the image (the right-hand side) by $Z^{\prime}(\mathbf{i})$. Since $Z^{\prime}(\mathbf{i})$ is a finite successive $\mathbb{P}^{1}$-fibration, it is proper. The map $f_{\mathbf{i}}^{+}$is proper, as the product map

$$
\left(\eta_{\mathbf{i}} \times f_{\mathbf{i}}^{+}\right): Z(\mathbf{i}) \hookrightarrow Z^{\prime}(\mathbf{i}) \times \mathbf{I}\left(i_{1}\right) \cdots \mathbf{I}\left(i_{\ell}\right) \mathbf{Q}_{G}\left(t_{\beta}\right)=Z^{\prime}(\mathbf{i}) \times \mathbf{Q}_{G}(e)
$$

is a closed immersion. In view of the isomorphism $\mathbf{Q}_{G}\left(t_{\beta}\right) \cong \mathbf{Q}_{G}(e)$, we transplant $\mathbf{Q}_{G}(e)_{<m}$ to $\mathbf{Q}_{G}\left(t_{\beta}\right)_{<m}$.

Claim A. For each closed point $x \in \mathbf{Q}_{G}(e)_{\leq m}$, the scheme

$$
\left(f_{\mathbf{i}}^{+}\right)^{-1}(x) \backslash\left(\left(f_{\mathbf{i}}^{+}\right)^{-1}(x) \cap\left(\mathbf{I}\left(i_{1}\right) \times{ }^{\mathbf{I}} \mathbf{I}\left(i_{2}\right) \times \times^{\mathbf{I}} \cdots \times \times^{\mathbf{I}} \mathbf{Q}_{G}\left(t_{\beta}\right)_{<m}\right)\right) \subset\left(f_{\mathbf{i}}^{+}\right)^{-1}(x)
$$

is a closed subscheme that is zero-dimensional. In other words, it is a finite union of points (potentially an empty set).

Proof. For each sequence $\left(j_{1}, \ldots, j_{s}\right) \in \mathrm{I}_{\text {af }}^{s}\left(s \in \mathbb{Z}_{>0}\right)$ and $v \leq_{\frac{\infty}{2}} e$ such that $\ell^{\frac{\infty}{2}}(v)=\ell$, the image of the map

$$
f: \mathbf{I}\left(j_{1}\right) \times{ }^{\mathbf{I}} \mathbf{I}\left(j_{2}\right) \times{ }^{\mathbf{I}} \cdots \times{ }^{\mathbf{I}} \mathbf{I}\left(j_{s}\right) \times{ }^{\mathbf{I}} \mathbf{Q}_{G}(v) \longrightarrow \mathbf{Q}_{G}^{\text {rat }}
$$


induced by the multiplication is a union of I-orbits $\mathbb{O}\left(v^{\prime}\right)$ with $\ell^{\frac{\infty}{2}}\left(v^{\prime}\right) \geq \ell-s$ (as we have $\ell^{\frac{\infty}{2}}\left(s_{i} w\right) \in$ $\left\{\ell^{\frac{\infty}{2}}(w) \pm 1\right\}$ for each $i \in \mathrm{I}_{\mathrm{af}}$ and $w \in W_{\text {af }}$ by [74, Lecture 13, Proposition $\left.\ell_{s}\right]$ ). In addition, if the image of the map $f$ contains $\mathbb{O}\left(v^{\prime}\right)$ for $\ell^{\frac{\infty}{2}}\left(v^{\prime}\right)=\ell-s$, then $f$ is an isomorphism along $\mathbb{O}\left(v^{\prime}\right)$ (as the isomorphism between open subsets). By collecting these for I-orbits in the closed subset $\mathbf{Q}_{G}\left(t_{\beta}\right) \backslash \mathbf{Q}_{G}\left(t_{\beta}\right)_{<m}$ of $\mathbf{Q}_{G}\left(t_{\beta}\right)$ in the construction of the (proper) map $f_{\mathbf{i}}^{+}$, we conclude the result.

We return to the proof of Proposition 4.26. By Claim A, we deduce that

$$
\overline{\left(f_{\mathbf{i}}^{+}\right)^{-1}(x) \cap \mathbf{I}\left(i_{1}\right) \times \mathbf{I} \mathbf{I}\left(i_{2}\right) \times \mathbf{I} \cdots \times \times^{\mathbf{I}} \mathbf{Q}_{G}\left(t_{\beta}\right)_{<m}} \subset\left(f_{\mathbf{i}}^{+}\right)^{-1}(x)
$$

is a union of connected components of $\left(f_{\mathbf{i}}^{+}\right)^{-1}(x)$ for each closed point $x \in \mathbf{Q}_{G}(e)_{\leq m}$.

Requiring regular functions on $\left(f_{\mathbf{i}}^{+}\right)^{-1}\left(\mathbf{Q}_{G}(e)_{\leq m}\right)$ to be constant along all the fibres yields sections in $\left(f_{\mathbf{i}}^{+}\right)_{*} \mathcal{O}_{Z(\mathbf{i})}$. From this (for arbitrary $t_{\beta} \leq_{\frac{\infty}{2}} e$ and $\mathbf{i}$ ) and the induction hypothesis, we conclude that $\pi^{-1}\left(\mathbf{Q}_{G}(e)_{\leq m}\right) \subset Z$ is a union of proper schemes over $\mathbf{Q}_{G}(e)_{\leq m}$ that contains a Zariski open subset $\mathbf{Q}_{G}(e)_{<m}$. In view of Corollary 3.39 (when char $\mathbb{K}>0$ ) or Proposition A.1 (when char $\mathbb{K}=0$; compare [51, Theorem A]), we deduce that

$$
(Z \supset) \pi^{-1}\left(\mathbf{Q}_{G}(e)_{\leq m}\right) \rightarrow \mathbf{Q}_{G}(e)_{\leq m}
$$

defines an isomorphism as schemes (as $\pi^{-1}\left(\mathbf{Q}_{G}(e)_{\leq m}\right) \rightarrow \mathbf{Q}_{G}(e)_{\leq m}$ is finite bijective and birational; compare [77, Section 02LQ]). Therefore, induction on $m$ proceeds and we conclude $Z \cong \mathbf{Q}_{G}(e)$ as schemes. Thus we obtain a morphism $\mathbf{Q}_{G}(e) \rightarrow X_{n}$ of schemes.

Rearranging $\mathbf{Q}_{G}(e)$ by the right $Q^{\vee}$-translations, we deduce a morphism $\mathbf{Q}_{G}^{\text {rat }} \rightarrow \mathfrak{X}$ as ind-schemes. This yields a continuous morphism $h_{\mathbf{Q}_{G}^{\text {rat }}} \rightarrow h_{\mathfrak{X}}$. Therefore, $h_{\mathbf{Q}_{G}^{\text {rat }}}$ is an initial object in the category of sheaves on $Z a r_{\mathbb{K}}$ ind-representable by strict ind-schemes that admits a continuous morphism from $\mathcal{Q}$, as required.

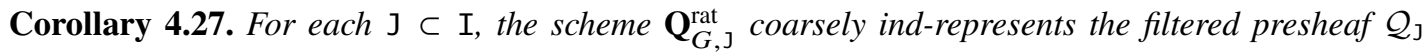
defined by

$$
A f f_{\mathbb{K}}^{o p} \ni R \mapsto \mathcal{Q}_{\mathrm{J}}(R):=G(R((z))) /\left(H(R) \cdot\left[P_{\mathrm{J}}, P_{\mathrm{J}}\right](R((z)))\right) \in \text { Sets. }
$$

Proof. By construction, we have a continuous morphism of presheaves $\mathcal{Q} \rightarrow \mathcal{Q}_{\text {J }}$ (by transplanting subsheaves $\mathcal{Q}_{n}$ to $\mathcal{Q}_{\text {J }}$ via this map). Thus, the coarse ind-representability of $\mathbf{Q}_{G}^{\text {rat }}$ implies that the maximal ind-scheme $X$ obtained by gluing points of $\mathbf{Q}_{G}^{\text {rat }}$ admits a continuous morphism $\mathcal{Q}_{\mathrm{J}} \rightarrow h_{X}$ that coarsely ind-represents the filtered presheaf $\mathcal{Q}_{\mathrm{J}}$. Every two rational functions on $\mathbf{Q}_{G}(w)\left(w \in W_{\mathrm{af}}\right)$ are distinguished by some pair of $\mathbb{K}$-valued points (Corollary 4.19). Since we have $\mathcal{Q}_{\mathrm{J}}(\mathbb{K})=\mathbf{Q}_{G, \mathrm{~J}}^{\text {rat }}(\mathbb{K})$, we conclude that $X=\mathbf{Q}_{G, \mathrm{~J}}^{\text {rat }}$.

\subsection{The properties of the schemes $Q_{j}^{\prime}(v, w)$}

In the rest of this section, we assume char $\mathbb{K} \neq 2$.

Lemma 4.28. Set $\mathrm{J} \subset \mathrm{I}$. For each $\beta \in Q_{+}^{\vee}$, the set of $\mathbb{K}$-valued points of $Q_{\mathrm{J}}^{\prime}(\beta, e)_{\mathbb{K}}$ is in bijection with the collection $\left\{u_{\lambda}(z)\right\}_{\lambda \in P_{\mathrm{J},+}}$ such that

$\circ u_{\lambda}(z) \in V(\lambda)_{\mathbb{K}} \otimes \bigoplus_{j=0}^{-\left\langle w_{0} \beta, \lambda\right\rangle} \mathbb{K} z^{j}$ and

○ for each $\lambda, \mu \in P_{+}$, we have $\eta_{\lambda, \mu}\left(u_{\lambda}(z) \otimes u_{\mu}(z)\right)=u_{\lambda+\mu}(z)$.

Proof. We have

$$
\mathbf{Q}_{G, \mathrm{~J}}(e)_{\mathbb{K}}=\left(\mathbf{Q}_{G, \mathrm{~J}}^{\mathrm{rat}}\right)_{\mathbb{K}} \cap \prod_{i \in \mathbb{I} \backslash \mathrm{J}} \mathbb{P}\left(V\left(\varpi_{i}\right)_{\mathbb{K}} \otimes \mathbb{K}[[z]]\right) \subset \prod_{i \in \mathrm{I} \backslash \mathrm{J}} \mathbb{P}\left(V\left(\varpi_{i}\right)_{\mathbb{K}} \otimes \mathbb{K}((z))\right)
$$


by Theorem 4.18. By the symmetry of the construction of $Q_{j}^{\prime}(\beta, e)$ in terms of $\theta$ (compare Remark 3.31), we conclude that

$$
\widehat{Q}_{J}^{\prime}(\beta, e)_{\mathbb{K}}=\left(\mathbf{Q}_{G, J}^{\mathrm{rat}}\right)_{\mathbb{K}} \cap \prod_{i \in \mathbb{I} \backslash \mathrm{J}} \mathbb{P}\left(V\left(\varpi_{i}\right)_{\mathbb{K}} \otimes \mathbb{K}[[z]]\right) \cap \prod_{i \in \mathrm{I} \backslash \mathrm{J}} \mathbb{P}\left(V\left(\varpi_{i}\right)_{\mathbb{K}} \otimes \mathbb{K}\left[\left[z^{-1}\right]\right] z^{-\left\langle\beta, w_{0} \varpi_{i}\right\rangle}\right)
$$

inside $\prod_{i \in \mathrm{I} \backslash \mathrm{J}} \mathbb{P}\left(V\left(\varpi_{i}\right)_{\mathbb{K}} \otimes \mathbb{K}\left[\left[z, z^{-1}\right]\right]\right)$, which is our degree bound. In view of this, it suffices to remember that the second condition is the same as the Plücker relation that defines $G((z)) / H(\mathbb{K})$. $[P(\mathrm{~J}), P(\mathrm{~J})]((z))$ in the last two paragraphs of the proof of Proposition 4.16.

Set $w, v \in W_{\text {af }}$ and $\mathrm{J} \subset \mathrm{I}$. For each $\lambda \in P_{\mathrm{J},+}$, we have a line bundle $\mathcal{O}_{Q_{\mathrm{J}}^{\prime}(v, w)}(\lambda)$ on $\mathbb{Q}_{\mathrm{J}}^{\prime}(v, w)$ such that we have a map

$$
R_{w}^{v}(\mathrm{~J}, \lambda) \rightarrow \Gamma\left({Q_{\mathrm{J}}^{\prime}}^{\prime}(v, w), \mathcal{O}_{Q_{\jmath}^{\prime}(v, w)}(\lambda)\right)
$$

that commutes with the multiplications. This yields $\mathcal{O}_{\mathscr{Q}_{\jmath}^{\prime}}(v, w)(\lambda)$ for each $\lambda \in P_{\jmath}$ by tensor products.

Lemma 4.29. For each $w, v \in W_{\mathrm{af}}$, the line bundle $\mathcal{O}_{\mathscr{Q}_{j}^{\prime}(v, w)}(\lambda)$ is very ample if $\left\langle\alpha_{i}^{\vee}, \lambda\right\rangle>0$ for every $i \in \mathrm{I} \backslash \mathrm{J}$.

Proof. We can assume that $\mathbb{Q}_{\jmath}^{\prime}(v, w) \neq \emptyset$ without the loss of generality.

By Lemma 4.1, we have

$$
\widehat{Q}_{\mathrm{J}}^{\prime}(v, w) \hookrightarrow \prod_{i \in \mathrm{I} \backslash \mathrm{J}} \mathbb{P}_{\mathbb{Z}}\left(R_{w}^{v}\left(\varpi_{i}\right)^{\vee}\right)
$$

From this and again by Lemma 4.1, we deduce that the following diagram is commutative:

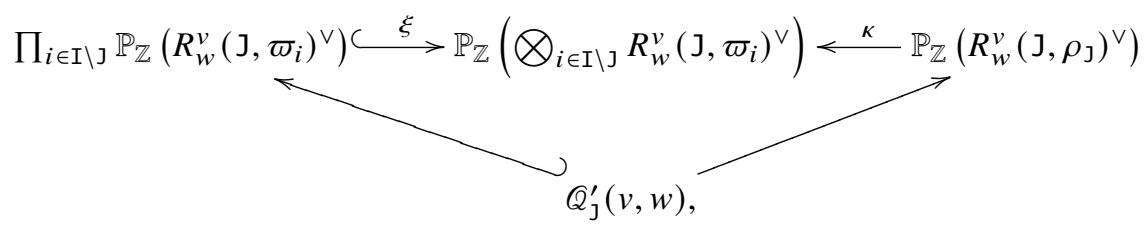

where $\xi$ is the Veronese embedding and $\kappa$ is induced from the multiplication map. The ring $R_{w}^{v}(J)$ is reduced by Corollary 4.10. Since $\left\langle\alpha_{i}^{\vee}, \rho_{\mathrm{J}}\right\rangle>0$ for every $i \in \mathrm{I} \backslash \mathrm{J}$ and the multiplication maps are surjective, a nonzero element of $R_{w}^{v}\left(\mathrm{~J}, \rho_{\mathrm{J}}\right)$ is not supported in the irrelevant locus. It follows that $R_{w}^{v}\left(\mathrm{~J}, \rho_{\mathrm{J}}\right) \subset H^{0}\left({Q^{\prime}}_{\mathrm{J}}(v, w), \mathcal{O}_{Q_{\mathrm{Q}}^{\prime}(v, w)}\left(\rho_{\mathrm{J}}\right)\right)$ and it is spanned by a product of linear functions that separates closed points of $\mathscr{Q}_{j}^{\prime}(v, w)$. In particular, $\mathcal{O}_{Q_{j}^{\prime}(v, w)}\left(\rho_{\mathrm{J}}\right)$ is very ample. Since we have embeddings

$$
R_{w}^{v}\left(\mathrm{~J}, \rho_{\mathrm{J}}\right) \subset R_{w}^{v}(\mathrm{~J}, \lambda) \subset H^{0}\left(Q_{\mathrm{J}}^{\prime}(v, w), \mathcal{O}_{Q_{\mathrm{J}}^{\prime}(v, w)}(\lambda)\right)
$$

obtained through multiplications corresponding to the duals of extremal weight vectors (so that the image is base-point free along an $\left(H \times \mathbb{G}_{m}\right)$-stable Zariski open neighbourhood of each $\left(H \times \mathbb{G}_{m}\right)$-fixed point of a projective variety), we conclude that $\mathcal{O}_{Q_{\jmath}^{\prime}(v, w)}(\lambda)$ is also very ample, as required.

Theorem 4.30. For each $w=u t_{\beta^{\prime}} \in W_{\mathrm{af}}\left(u \in W, \beta^{\prime} \in Q^{\vee}\right)$ and $\beta \in Q_{+}^{\vee}$, we have an isomorphism $\mathbb{Q}^{\prime}(\beta, w)_{\mathbb{C}} \cong \mathbb{Q}\left(\beta-\beta^{\prime}, u\right)$ as varieties. Moreover, $\mathbb{Q}^{\prime}(\beta, w)_{\mathbb{K}}$ is irreducible, and its dimension is given as

$$
\operatorname{dim} \mathscr{Q}^{\prime}(\beta, w)_{\mathbb{K}}=2\left\langle\beta-\beta^{\prime}, \rho\right\rangle+\operatorname{dim} \mathscr{B}(u) .
$$

Proof. By Lemma 4.7, we know that $\left(R_{v}^{\beta}\right)_{\mathbb{K}}$ is a quotient of $\left(R_{w}^{\beta}\right)_{\mathbb{K}}$ for $v \in W_{\text {af }}$ such that $v \leq_{\frac{\infty}{2}} w$. Hence, we have $Q^{\prime}(\beta, w)_{\mathbb{K}} \cap \mathbf{Q}_{G}(v)_{\mathbb{K}}=\mathscr{Q}^{\prime}(\beta, v)_{\mathbb{K}}$. By Lemma 4.6, the scheme $\mathbb{Q}^{\prime}(\beta, w)_{\mathbb{K}} \cap \mathbb{O}(v)_{\mathbb{K}}$ is 
isomorphic to $Q^{\prime}\left(\beta-\gamma, u^{\prime}\right)_{\mathbb{K}} \cap \mathbb{O}\left(u^{\prime}\right)_{\mathbb{K}}$, where $v=u^{\prime} t_{\gamma}\left(u^{\prime} \in W, \gamma \in Q_{+}^{\vee}\right)$. By Lemma 4.28 and Theorem 2.8, we have the following:

$\circ\left(Q^{\prime}(\beta, w)_{\mathbb{K}} \cap \mathbb{O}(v)_{\mathbb{K}}\right)=\left(Q^{\prime}(\beta, v)_{\mathbb{K}} \cap \mathbb{O}(v)_{\mathbb{K}}\right)$ for each $v \in W_{\text {af }}$ such that $v \leq_{\frac{\infty}{2}} w$.

$\circ$ The variety $\left(Q^{\prime}(\beta, w)_{\mathbb{R}} \cap \mathbb{O}(v)_{\mathbb{K}}\right)$ is irreducible for every $v \in W_{\mathrm{af}}$.

We have

$$
\operatorname{dim}\left(\mathbb{Q}^{\prime}(\beta, w)_{\mathbb{C}} \cap \mathbb{O}(v)_{\mathbb{C}}\right)=\operatorname{dim} \mathscr{Q}\left(\beta-\gamma, u^{\prime}\right)=2\langle\beta-\gamma, \rho\rangle+\operatorname{dim} \mathscr{B}\left(u^{\prime}\right)
$$

for $v=u^{\prime} t_{\gamma}\left(u^{\prime} \in W, \gamma \in Q_{+}^{\vee}\right)$ by Lemma 4.28 and Theorem 2.7 (whenever $\left.\mathbb{Q}^{\prime}(\beta, w)_{\mathbb{C}} \cap \mathbb{O}(v)_{\mathbb{C}} \neq \emptyset\right)$. In addition, Lemma 4.3 implies

$\circ \operatorname{dim} \mathbb{Q}^{\prime}(\beta, w)_{\mathbb{C}}=\operatorname{dim} \mathbb{Q}^{\prime}(\beta, w)_{\mathbb{K}}$.

In particular, we have the desired dimension formula if $\mathbb{Q}^{\prime}(\beta, w)_{\mathbb{K}}$ is irreducible with its Zariski open dense subset $\left(\mathscr{Q}^{\prime}(\beta, w)_{\mathbb{K}} \cap \mathbb{O}(w)_{\mathbb{K}}\right)$.

Since $\mathbb{Q}(\beta, w)$ and $\mathbb{Q}^{\prime}(\beta, w)_{\mathbb{C}}$ share an open subset and the former is irreducible, we have $\mathbb{Q}(\beta, w)=$ $\mathbb{Q}^{\prime}(\beta, w)_{\mathbb{C}}$ as closed subvarieties of $\left(\mathbf{Q}_{G}^{\text {rat }}\right)_{\mathbb{C}}$ if $\mathbb{Q}^{\prime}(\beta, w)_{\mathbb{C}}$ is irreducible.

Therefore, it suffices to prove that $Q^{\prime}(\beta, w)_{\mathbb{K}}$ is irreducible (with its Zariski open dense subset $\left.\left(Q^{\prime}(\beta, w)_{\mathbb{K}} \cap \mathbb{O}(w)_{\mathbb{R}}\right)\right)$. By Theorem 2.1 and Corollary 4.21, it suffices to prove that

$$
\left(\mathscr{Q}^{\prime}(\beta, w)_{\mathbb{K}} \cap \mathbb{O}(s w)_{\mathbb{K}}\right) \subset \overline{\left(Q^{\prime}(\beta, w)_{\mathbb{K}} \cap \mathbb{O}(w)_{\mathbb{K}}\right)}
$$

for every $w \in W_{\text {af }}$ and every reflection $s \in W_{\text {af }}$ such that $\ell^{\frac{\infty}{2}}(s w)=\ell^{\frac{\infty}{2}}(w)+1$ and $w_{0} t_{\beta} \leq_{\frac{\infty}{2}} s w \leq_{\frac{\infty}{2}} w$. Here $\mathbf{Q}_{G}(s v)_{\mathbb{K}} \subset \mathbf{Q}_{G}(v)_{\mathbb{K}}$ is an irreducible component of the boundary by Theorem 2.4(3) (compare Corollary 4.20). This boundary component is these cut out as (a part of) the zero of $\mathbf{v}_{v w_{0} \lambda}^{\vee} \in \mathbb{W}_{v w_{0}}(\lambda)^{\vee}$ $\left(\lambda \in P_{+}\right)$. Thus, we deduce that $\overline{\left(\widehat{Q}^{\prime}(\beta, w)_{\mathbb{K}} \cap \mathbb{O}(s w)_{\mathbb{K}}\right)}$ contains an irreducible component of

$$
\overline{\left(Q^{\prime}(\beta, w)_{\mathbb{K}} \cap \mathbb{O}(w)_{\mathbb{K}}\right)} \cap\{f=0\}
$$

for some single equation $f$ (an instance of $\mathbf{v}_{v w_{0} \lambda}^{\vee} \mathrm{s}$ ) if it is nonempty. By comparison of dimensions, this forces

$$
\overline{\left(Q^{\prime}(\beta, w)_{\mathbb{K}} \cap \mathbb{O}(w)_{\mathbb{K}}\right)} \cap\{f=0\} \cap \mathbb{O}(s w)_{\mathbb{K}} \subset\left(Q^{\prime}(\beta, w)_{\mathbb{K}} \cap \mathbb{O}(s w)_{\mathbb{K}}\right)
$$

to be an irreducible component if the left-hand side is nonempty. Consider the $\left(H \times \mathbb{G}_{m}\right)$-invariant curve $C$ that connects the (unique) $\left(H \times \mathbb{G}_{m}\right)$-fixed points $p_{s w}$ in $\mathbb{O}(s w)_{\mathbb{K}}$ and $p_{w}$ in $\mathbb{O}(w)_{\mathbb{K}}$. The curve $C$ is the closure of the orbit of a one-parameter unipotent subgroup action corresponding to a root in $\Delta_{\mathrm{af},+}$ applied to $p_{w}$. In particular, we have $C \subset \mathbb{O}(s w)_{\mathbb{K}} \sqcup \mathbb{O}(w)_{\mathbb{K}}$. By the degree bound (from above) offered by Lemma 4.28 , we conclude that $C \subset \overline{\left(Q^{\prime}(\beta, w)_{\mathbb{K}} \cap \mathbb{O}(w)_{\mathbb{K}}\right)}$. Therefore, the left-hand side of formula (4.13) is nonempty. Hence, the irreducibility of $\left(Q^{\prime}(\beta, w)_{\mathbb{R}} \cap \mathbb{O}(s w)_{\mathbb{K}}\right)$ forces

$$
\overline{\left(Q^{\prime}(\beta, w)_{\mathbb{K}} \cap \mathbb{O}(w)_{\mathbb{K}}\right)} \cap\{f=0\} \cap \mathbb{O}(s w)_{\mathbb{K}}=\left(Q^{\prime}(\beta, w)_{\mathbb{K}} \cap \mathbb{O}(s w)_{\mathbb{K}}\right) .
$$

Therefore, we conclude formula (4.12). This implies $\mathbb{Q}\left(\beta-\beta^{\prime}, u\right)=\mathbb{Q}^{\prime}(\beta, w)_{\mathbb{C}}$ as an irreducible (reduced) closed subvariety of $\left(\mathbf{Q}_{G}^{\text {rat }}\right)_{\mathbb{C}}$, and $Q^{\prime}(\beta, w)_{\mathbb{K}}$ is irreducible in general. Its dimension $\operatorname{dim} Q(\beta-$ $\left.\beta^{\prime}, u\right)$ comes from the dimension of $\left(Q^{\prime}(\beta, w)_{\mathbb{K}} \cap \mathbb{O}(w)_{\mathbb{K}}\right)$, which is a Zariski open dense subset of $Q^{\prime}(\beta, w)_{\mathbb{R}}$.

Corollary 4.31. For each $w, v \in W_{\mathrm{af}}$, the dimension of an irreducible component of $\mathbb{Q}^{\prime}(v, w)_{\mathbb{K}}$ is $\ell^{\frac{\infty}{2}}(v)-\ell^{\frac{\infty}{2}}(w)$ if $Q^{\prime}(v, w)_{\mathbb{K}} \neq \emptyset$ (which is equivalent to $v \leq_{\frac{\infty}{2}} w$ by Corollary 4.21). In particular, $Q^{\prime}(v, w) \mathbb{K}$ is equidimensional. 
Proof. The case where $v=w_{0} t_{\beta}$ with $\beta \in Q^{\vee}$ follows from Theorem 4.30. We have $Q^{\prime}(w, w)_{\mathbb{K}}=\operatorname{Spec} \mathbb{K}$ $\left(w \in W_{\text {af }}\right)$, since $\operatorname{dim} \mathbb{W}_{w w_{0}}(\lambda)_{\mathbb{K}} \cap \mathbb{W}_{w w_{0}}^{-}(\lambda)_{\mathbb{K}}=1$ for every $\lambda \in P_{+}$by $P^{\text {af }}$-weight comparison.

Let $v=u t_{\beta}(u \in W)$. In view of the proof of Theorem 4.30, the variety $Q^{\prime}(v, w)_{\mathbb{K}}$ is obtained from $Q^{\prime}\left(w_{0} t_{\beta}, w\right)_{\mathbb{K}}$ by $\left(\ell\left(w_{0}\right)-\ell(u)\right)$-successive hyperplane cuts by the $\theta$-twists of the I-stable boundaries of $\mathbf{Q}_{G}(\bullet)_{\mathbb{K}}$ (compare Remark 3.31). Each of these hyperplane cuts lowers the dimension of an irreducible component by at most 1 if the intersection is nonempty. Since $\ell\left(w_{0}\right)-\ell(u)=\ell^{\frac{\infty}{2}}\left(w_{0} t_{\beta}\right)-\ell^{\frac{\infty}{2}}(v)$, the dimension inequality $\geq$ always holds.

Every irreducible component of $\mathcal{Q}^{\prime}(v, w)_{\mathbb{K}}$ is $\left(H \times \mathbb{G}_{m}\right)$-stable by construction. Since we have only one $\left(H \times \mathbb{G}_{m}\right)$-fixed point in each $\mathbf{I}$-orbit of $\left(\mathbf{Q}_{G}^{\text {rat }}\right)_{\mathbb{K}}$, all but one $\left(H \times \mathbb{G}_{m}\right)$-fixed point of $\mathbf{Q}_{G}(w)_{\mathbb{K}}$ $\left(w \in W_{\mathrm{af}}\right)$ lie in the boundary. Thus, if an irreducible component of $\mathscr{Q}^{\prime}(v, w)_{\mathbb{K}}$ does not meet any $\mathbf{I}$ stable boundary divisors of $\mathbf{Q}_{G}(w)_{\mathbb{K}}$ or the $\theta$-twists of any I-stable boundary divisors of $\mathbf{Q}_{G}\left(v w_{0}\right)_{\mathbb{K}}$, then such an irreducible component does not contain an $\left(H \times \mathbb{G}_{m}\right)$-fixed point unless $v=w$. This is a contradiction (of the properness of $Q^{\prime}(v, w)_{\mathbb{K}}$ ). Hence, every irreducible component of $\mathbb{Q}^{\prime}(v, w)_{\mathbb{K}}$ meets its boundary cut out by an I-stable boundary divisor of $\mathbf{Q}_{G}(w)_{\mathbb{K}}$ or the $\theta$-twist of an I-stable boundary divisor of $\mathbf{Q}_{G}\left(v w_{0}\right)_{\mathbb{K}}$.

Assume the contrary to deduce a contradiction. Then we have $\mathbb{Q}^{\prime}(v, w)_{\mathbb{K}}$, whose irreducible components have dimension $\left(\ell^{\frac{\infty}{2}}(v)-\ell^{\frac{\infty}{2}}(w)\right)$, but one of its irreducible components is in fact contained in $Q^{\prime}\left(v^{\prime}, w^{\prime}\right)_{\mathbb{K}}$ with $Q^{\prime}\left(v^{\prime}, w^{\prime}\right)_{\mathbb{K}} \subsetneq Q^{\prime}(v, w)_{\mathbb{K}}$ for $v^{\prime}, w^{\prime} \in W_{\text {af }}$, and hence it gives an irreducible component of $Q^{\prime}\left(v^{\prime}, w^{\prime}\right)_{\mathbb{K}}$ with dimension $>\left(\ell^{\frac{\infty}{2}}\left(v^{\prime}\right)-\ell^{\frac{\infty}{2}}\left(w^{\prime}\right)\right)$. From this, we can lower the dimension of an irreducible component of $Q^{\prime}\left(v^{\prime}, w^{\prime}\right)_{\mathbb{K}}$ by intersecting with a divisor to raise $v^{\prime}$ or lower $w^{\prime}$ successively to reach the case $v^{\prime}=w^{\prime}$ (as in the second paragraph). Since the dimension drops at most 1 in each step, we find that $\operatorname{dim} Q^{\prime}(w, w)_{\mathbb{K}}>0$ for some $w \in W_{\text {af }}$. This is a contradiction, and hence this case does not happen.

These imply that the dimension equality always holds, as required.

Remark 4.32. The analogous assertions from Theorem 4.30 and Corollary 4.31 hold for the case where $\mathrm{J} \subsetneq \mathrm{I}$ (compare Corollaries 5.21 and 5.22).

Theorem 4.33. For each $w, v \in W_{\mathrm{af}}$, $\mathrm{J} \subset \mathrm{I}$ and $\lambda \in P_{\mathrm{J},+}$, we have

$$
H^{i}\left({Q_{\jmath}^{\prime}}^{\prime}(v, w)_{\mathbb{K}}, \mathcal{O}_{Q_{\jmath}^{\prime}(v, w)_{\mathbb{K}}}(\lambda)\right) \cong \begin{cases}R_{w}^{v}(\lambda)_{\mathbb{K}}, & i=0, \lambda \in P_{\mathrm{J},++}, \\ \{0\}, & i \neq 0 .\end{cases}
$$

Moreover, if $w^{\prime}, v^{\prime} \in W_{\mathrm{af}}$ satisfies $\mathcal{Q}_{\mathrm{J}}^{\prime}\left(v^{\prime}, w^{\prime}\right) \subset{Q_{\mathrm{J}}^{\prime}}^{\prime}(v, w)$ and $\lambda \in P_{\mathrm{J},+}$, then the restriction map induces a surjection

$$
H^{0}\left(Q_{J}^{\prime}(v, w)_{\mathbb{R}}, \mathcal{O}_{Q_{J}^{\prime}(v, w)_{\mathbb{K}}}(\lambda)\right) \longrightarrow H^{0}\left(Q_{J}^{\prime}\left(v^{\prime}, w^{\prime}\right)_{\mathbb{K}}, \mathcal{O}_{Q_{J}^{\prime}\left(v^{\prime}, w^{\prime}\right)_{\mathbb{K}}}(\lambda)\right)
$$

Remark 4.34. The strict dominance condition in Theorem 4.33 cannot be removed naively. For $G=$ $S L(3), \mathbb{K}=\mathbb{C}$ and $w=e$, we have

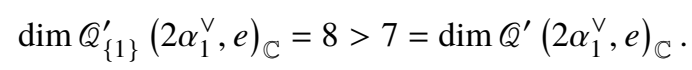

This results in the noninjectivity of the pullback map

$$
H^{0}\left({Q^{\prime}}_{\{1\}}\left(2 \alpha_{1}^{\vee}, e\right)_{\mathbb{C}}, \mathcal{O}_{Q_{\{1\}}^{\prime}\left(2 \alpha_{1}^{\vee}, e\right)_{\mathbb{C}}}\left(3 \varpi_{1}\right)\right) \longrightarrow H^{0}\left(Q^{\prime}\left(2 \alpha_{1}^{\vee}, e\right)_{\mathbb{C}}, \mathcal{O}_{Q^{\prime}}\left(2 \alpha_{1}^{\vee}, e\right)_{\mathbb{C}}\left(3 \varpi_{1}\right)\right),
$$

where the left-hand side is $R_{e}^{2 \alpha_{1}^{\vee}}\left(3 \varpi_{1}\right)_{\mathbb{C}}=R_{e}^{2 \alpha_{1}^{\vee}}\left(\{1\}, 3 \varpi_{1}\right)_{\mathbb{C}} \cong S^{3}\left(\mathbb{C}^{3} \oplus \mathbb{C}^{3} z \oplus \mathbb{C}^{3} z^{2}\right)^{\vee}$ and the righthand side is its quotient by the $G$-invariants of $\left(\mathbb{C}^{3} \otimes \mathbb{C}^{3} z \otimes \mathbb{C}^{3} z^{2}\right)^{\vee} \subset S^{3}\left(\mathbb{C}^{3} \oplus \mathbb{C}^{3} z \oplus \mathbb{C}^{3} z^{2}\right)^{\vee}$. In fact, 
the ring $\left(R_{e}^{2 \alpha_{1}^{\vee}}\right)_{\mathbb{C}}$ is reduced but not integral, and the extra irreducible component is contained in the irrelevant locus.

Proof of Theorem 4.33. We first observe that it is enough to show the surjectivity part of the assertion only for the $w=w^{\prime}$ case, since the $v=v^{\prime}$ case follows by applying $\theta$ (and the rest of the cases follow by repeated applications of the two cases).

Since $\mathcal{O}(\mathrm{J}, w)_{\mathbb{F}_{p}} \subset \mathbf{Q}_{G, \mathrm{~J}}(w)_{\mathbb{F}_{p}}$ is affine, the union $Z$ of codimension one I-orbits contains the support of an ample divisor. The reduced union of the irreducible components of $Z$ affords a Weil divisor $D$ (by Corollary A.2). Since our Frobenius splitting of $\mathbf{Q}_{G, J}(w)_{\mathbb{F}_{p}}$ is $\mathbf{I}$-canonical and compatible with the I-orbit closures (Corollary 3.38), we deduce that $\mathbf{Q}_{G, \mathrm{~J}}(w)_{\mathbb{F}_{p}}$ is $D$-split in the sense of [15, Definition 3] (compare [75, Definition 1.2]). Each irreducible component of $Z$ intersects properly with $\mathscr{Q}_{\mathrm{J}}^{\prime}\left(v^{\prime}, w\right)_{\mathbb{F}_{p}} \subset \mathbb{Q}_{\mathrm{J}}^{\prime}(v, w)_{\mathbb{F}_{p}}\left(v^{\prime} \in W_{\mathrm{af}}\right)$ whenever the intersection is nonempty, by Corollary 4.31 (and Remark 4.32). Thus, we deduce that $Q_{j}^{\prime}(v, w)_{\mathbb{F}_{p}}$ admits a Frobenius $D$-splitting compatible with $Q_{j}^{\prime}\left(v^{\prime}, w\right)_{\mathbb{F}_{p}}\left(v^{\prime} \in W_{\text {af }}\right)$. Therefore, the cohomology vanishing part of the assertion and the surjectivity part (for $w^{\prime}=w$ ) follow from [75, Proposition 1.13 (ii)]. The cohomology vanishing part lifts to char $\mathbb{K}=0$ by [14, Proposition 1.6.2]. The surjectivity part lifts to char $\mathbb{K}=0$ by [14, Corollary 1.6.3] and Lemma 4.3.

It remains to calculate $H^{0}\left(Q_{\mathrm{J}}^{\prime}(v, w)_{\mathbb{K}}, \mathcal{O}_{Q_{\mathrm{J}}^{\prime}(v, w)_{\mathbb{K}}}(\lambda)\right)$ for each $\lambda \in P_{\mathrm{J},++}$. Here $H^{0}\left(Q_{\jmath}^{\prime}(v, w)_{\mathbb{K}}, \mathcal{O}_{Q_{j}^{\prime}(v, w)_{\mathbb{K}}}(\lambda)\right)$ is obtained as the degree $\lambda$ part of the (graded) normalisation of the ring $\left(R_{w}^{v}(\mathrm{~J})\right)_{\mathbb{K}^{*}}$. For each prime $p$, our ring $\left(R_{w}^{v}(\mathrm{~J})\right)_{\mathbb{F}_{p}}$ is weakly normal by Lemma 4.7. Let us consider the $P_{\mathrm{J},+}$-graded quotient $R^{\prime}=\bigoplus_{\lambda \in P_{\mathrm{J},+}} R^{\prime}(\lambda)$ of $R_{w}^{v}(\mathrm{~J}, \lambda)_{\mathbb{F}_{p}}$ that annihilates all the irrelevant irreducible components. Since $R_{w}^{v}(\mathrm{~J})_{\mathbb{F}_{p}}$ is a reduced ring, we have $R_{w}^{v}(\mathrm{~J}, \lambda)_{\mathbb{F}_{p}}=R^{\prime}(\lambda)$ for $\lambda \in P_{\mathrm{J},++}$. The ring $R^{\prime}$ is generated by $\bigoplus_{i \in \mathrm{I} \backslash \mathrm{J}} R^{\prime}\left(\varpi_{i}\right)$, because $R_{w}^{v}(\mathrm{~J})_{\mathbb{F}_{p}}$ is. We have

$$
H^{0}\left(Q_{\jmath}^{\prime}(v, w)_{\mathbb{F}_{p}}, \mathcal{O}_{Q_{\jmath}^{\prime}(v, w)_{\mathbb{F}_{p}}}(\lambda)\right) \cong R_{w}^{v}(J, \lambda)_{\mathbb{F}_{p}}
$$

for sufficiently large $\lambda \in P_{\mathrm{J},+}$ (see, e.g., [37, II Excecise 5.9]). In other words, formula (4.14) holds for $m \lambda$, where $\lambda \in P_{\mathrm{J},++}$ is arbitrary and $m \gg 0$.

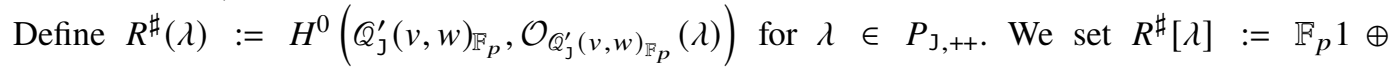
$\bigoplus_{m \geq 1} R^{\sharp}(m \lambda)$ and $R^{\prime}[\lambda]:=\bigoplus_{m \geq 0} R^{\prime}(m \lambda) \subset R^{\prime}$ for $\lambda \in P_{\mathrm{J},++}$. Both are naturally rings. Then we have a ring extension $R^{\prime}[\lambda] \subset R^{\sharp}[\lambda]$ such that their degree $m \gg 0$ parts are the same.

We prove $R^{\prime}(\lambda)=R^{\sharp}(\lambda)$. The ring $R^{\prime}[\lambda]$ is weakly normal, as we have an inclusion $R^{\prime}[\lambda] \hookrightarrow$ $R_{w}^{v}(\mathrm{~J})_{\mathbb{F}_{p}}$ (and the reasoning that $R_{w}^{v}(\mathrm{~J})_{\mathbb{F}_{p}}$ is weakly normal equally applies to $R^{\prime}[\lambda]$ ). Since it is generated by $R^{\prime}(\lambda)$, we deduce that the rings $R^{\prime}[\lambda]$ and $R^{\sharp}[\lambda]$ share the same spectrum (the irrelevant locus of $R^{\prime}[\lambda]$ is one point, as it is a $\mathbb{Z}_{\geq 0}$-graded ring, and other points are just the same). This forces $R^{\prime}(\lambda)=R^{\sharp}(\lambda)$ by the weak normality of $R^{\prime}[\lambda]$.

This yields the $H^{0}$-part of the assertion for char $\mathbb{K}>0$ through the extension of scalars. The $H^{0}$-part of the assertion for char $\mathbb{K}=0$ is obtained by taking the generic specialisation of the base scheme Spec $\mathbb{Z}$ of $Q_{j}^{\prime}(v, w)$.

Corollary 4.35. Set $w, v \in W_{\text {af }}$ and $\mathrm{J} \subset \mathrm{I}$. Assume that the map $\Pi_{\mathrm{J}}: \mathbb{Q}^{\prime}(v, w)_{\mathbb{K}} \rightarrow \mathbb{Q}_{\mathrm{J}}^{\prime}(v, w)_{\mathbb{K}}$ defined through the projective coordinate ring is surjective. We have

$$
\mathbb{R}^{>0}\left(\Pi_{\mathrm{J}}\right)_{*} \mathcal{O}_{Q^{\prime}(v, w)_{\mathbb{K}}} \cong\{0\} \quad \text { and } \quad\left(\Pi_{\mathrm{J}}\right)_{*} \mathcal{O}_{Q^{\prime}(v, w)_{\mathbb{K}}} \cong \mathcal{O}_{Q^{\prime}}(v, w)_{\mathbb{K}} .
$$

Remark 4.36. Since the map $\mathbf{Q}_{G}^{\text {rat }} \rightarrow \mathbf{Q}_{G, J}^{\text {rat }}$ is surjective, we can replace $v$ with $v t_{\beta}$ for some $\beta \in$ $\sum_{j \in \mathrm{J}} \mathbb{Z}_{\geq 0} \alpha_{j}^{\vee}$ to obtain a surjection

$$
\Pi_{\mathrm{J}}:{Q^{\prime}}^{\prime}\left(v t_{\beta}, w\right)_{\mathbb{K}} \rightarrow Q_{\mathrm{J}}^{\prime}\left(v t_{\beta}, w\right)_{\mathbb{K}}=Q_{\mathrm{J}}^{\prime}(v, w)_{\mathbb{K}}
$$


For $v=w_{0}$ and $w=e$, there is an optimal choice of $\beta$, which is sometimes referred to as the PetersonWoodward threshold ([79]).

Proof of Corollary 4.35. Thanks to Lemma 4.29 and [57, Lemma A.31], it suffices to prove

$$
H^{>0}\left(Q^{\prime}(v, w)_{\mathbb{K}}, \mathcal{O}_{Q^{\prime}}(v, w)_{\mathbb{K}}(\lambda)\right) \cong\{0\}
$$

and

$$
H^{0}\left(Q^{\prime}(v, w)_{\mathbb{K}}, \mathcal{O}_{Q^{\prime}(v, w)_{\mathbb{K}}}(\lambda)\right) \cong H^{0}\left(Q_{\jmath}^{\prime}(v, w)_{\mathbb{K}}, \mathcal{O}_{Q_{j}^{\prime}(v, w)_{\mathbb{K}}}(\lambda)\right)
$$

for each $\lambda \in P_{\mathrm{J},++}$. The $H^{>0}$-part of the assertion follow from Theorem 4.33. The $H^{0}$-part of the assertion follows as in the latter part of the proof of Theorem 4.33, since the surjectivity of $\Pi_{\mathrm{J}}$ guarantees that every nonzero element of $R_{w}^{v}(\lambda)_{\mathbb{F}_{p}}$ is supported outside of the irrelevant locus by pullback, and that the (graded) normalisation of the ring $R_{w}^{v}(\mathrm{~J})_{\mathbb{F}_{p}}$ is the degree $P_{\mathrm{J},+}$ part of the (graded) normalisation of the ring $\left(R_{w}^{v}\right)_{\mathbb{F}_{p}}$ by the degree reasons.

Corollary 4.37. Set $\lambda \in P_{+}$and $w, v \in W_{\mathrm{af}}$. We set $\mathrm{J}:=\left\{i \in \mathrm{I} \mid\left\langle\alpha_{i}^{\vee}, \lambda\right\rangle=0\right\}$. Assume that $\Pi_{\mathrm{J}}\left(\widehat{Q}^{\prime}(v, w)_{\mathbb{K}}\right)=\widehat{Q}_{\mathrm{J}}^{\prime}(v, w)_{\mathbb{K}}$. Then we have

$$
H^{0}\left(Q^{\prime}(v, w)_{\mathbb{K}}, \mathcal{O}_{Q^{\prime}(v, w)_{\mathbb{R}}}(\lambda)\right)^{\vee}=\mathbb{W}_{w w_{0}}(\lambda)_{\mathbb{K}} \cap \theta\left(\mathbb{W}_{v}\left(-w_{0} \lambda\right)_{\mathbb{K}}\right) .
$$

Proof. Combine Theorem 4.33 for $\mathbb{Q}_{\jmath}^{\prime}(v, w)_{\mathbb{K}}$ and Corollary 4.35 .

Lemma 4.38. Set $w, v \in W_{\mathrm{af}}$ and $\mathrm{J} \subset \mathrm{I}$. For each $i \in \mathrm{I}_{\mathrm{af}}$ such that $s_{i} w>_{\frac{\infty}{2}} w$ and $s_{i} v>_{\frac{\infty}{2}} v$, the variety $Q_{\mathrm{J}}^{\prime}(v, w)_{\mathbb{K}}$ is $B_{i}$-stable. In addition, we have a morphism

$$
\pi_{i}: S L(2, i) \times{ }^{B_{i}} Q_{\jmath}^{\prime}(v, w)_{\mathbb{K}} \rightarrow Q_{\jmath}^{\prime}\left(v, s_{i} w\right)_{\mathbb{R}}
$$

The map $\pi_{i}$ is a $\mathbb{P}^{1}$-fibration if $Q_{\jmath}^{\prime}(v, w)_{\mathbb{R}}$ is $S L(2, i)$-stable (this never happens under our assumption when $\mathrm{J}=\emptyset)$. In general, the fibre of $\pi_{i}$ is either a point or $\mathbb{P}^{1}$ if it is nonempty.

Proof. For each $\lambda \in P_{\mathrm{J},++}$, the module $\mathbb{W}_{v}\left(-w_{0} \lambda\right)_{\mathbb{K}}$ is $\mathbf{I}(i)$-stable by Corollary 3.16 and formula (2.2), and the module $\mathbb{W}_{w w_{0}}(\lambda)_{\mathbb{R}}$ is I-stable. In particular,

$$
\mathbb{W}_{w w_{0}}(\lambda)_{\mathbb{K}} \cap \theta\left(\mathbb{W}_{v}\left(-w_{0} \lambda\right)_{\mathbb{K}}\right) \equiv \mathbb{W}_{w w_{0}}(\lambda)_{\mathbb{K}} \cap \mathbb{W}_{v w_{0}}^{-}(\lambda)_{\mathbb{R}}
$$

is $B_{i}$-stable. It follows that the ring $R_{w}^{v}(\mathrm{~J})_{\mathbb{K}}$ is $B_{i}$-stable. Hence the scheme $\mathscr{Q}_{\jmath}^{\prime}(v, w)_{\mathbb{K}}$ is also $B_{i}$-stable.

The map $\pi_{i}$ is a $\mathbb{P}^{1}$-fibration if $Q_{j}^{\prime}(v, w)_{\mathbb{R}}$ is $S L(2, i)$-stable, since in this case we have $Q_{j}^{\prime}(\beta, w)_{\mathbb{R}}=$ $\mathcal{Q}_{\mathrm{J}}^{\prime}\left(\beta, s_{i} w\right)_{\mathbb{K}}$ (which in turn holds if and only if $\mathbb{W}_{s_{i} w w_{0}}(\lambda)_{\mathbb{K}}=\mathbb{W}_{w w_{0}}(\lambda)_{\mathbb{K}}$ for $\lambda \in P_{\mathrm{J},++}$, since $\mathbb{W}_{v w_{0}}^{-}(\lambda)_{\mathbb{K}}$ is $S L(2, i)$-stable; hence it never happens when $\mathrm{J}=\emptyset$, by Lemma 3.15). The fibre of the map $\pi_{i}$ is either a point or $\mathbb{P}^{1}$ if $Q_{j}^{\prime}(v, w)_{\mathbb{R}}$ is not $S L(2, i)$-stable, as the corresponding statement holds true for $\mathbf{Q}_{G, \mathrm{~J}}(w)_{\mathbb{K}} \subset \mathbf{Q}_{G, \mathrm{~J}}\left(s_{i} w\right)_{\mathbb{K}}$ by a set-theoretic consideration (and it carries over to any $B_{i}$-stable locus). Therefore, we conclude the result.

Proposition 4.39. Set $w, v \in W_{\mathrm{af}}$ and $\mathrm{J} \subset \mathrm{I}$. For each $i \in \mathrm{I}_{\mathrm{af}}$ such that $s_{i} w>_{\frac{\infty}{2}} w$ and $s_{i} v>_{\frac{\infty}{2}} v$, we have a surjective map

$$
\pi_{i}: S L(2, i) \times{ }^{B_{i}} Q_{\mathrm{J}}^{\prime}(v, w)_{\mathbb{K}} \rightarrow Q_{\mathrm{J}}^{\prime}\left(v, s_{i} w\right)_{\mathbb{K}}
$$

such that $\left(\pi_{i}\right)_{*} \mathcal{O}_{S L(2, i) \times{ }^{B_{i}} \mathscr{Q}_{j}^{\prime}(v, w)_{\mathbb{K}}} \cong \mathcal{O}_{\mathscr{Q}_{j}^{\prime}\left(v, s_{i} w\right)_{\mathbb{K}}}$ and

$$
\mathbb{R}^{>0}\left(\pi_{i}\right)_{*} \mathcal{O}_{S L(2, i) \times{ }^{B_{i} Q_{\mathfrak{J}}^{\prime}}(v, w)_{\mathbb{K}}} \cong\{0\} .
$$


Proof. For each $\lambda \in P_{\mathrm{J},++}$, the $\lambda$-graded component

$$
R_{w}^{v}(\mathrm{~J}, \lambda)^{\vee}=\mathbb{W}_{w w_{0}}(\lambda)_{\mathbb{Z}} \cap \theta^{*}\left(\mathbb{W}_{v}\left(-w_{0} \lambda\right)_{\mathbb{Z}}\right) \subset \mathbb{X}(\lambda)_{\mathbb{Z}}
$$

of the coordinate ring of $\widehat{Q}_{\jmath}^{\prime}(v, w)$ is obtained as the $\mathbb{Z}$-span of a subset of positive global basis of $\mathbb{W}_{w w_{0}}(\lambda)_{\mathbb{Z}}$.

We set $Q_{j}^{+}(v, w)_{\mathbb{K}}:=S L(2, i) \times{ }^{B_{i}} \mathscr{Q}_{j}^{\prime}(v, w)_{\mathbb{K}}$ for simplicity in what follows.

In view of [72, Theorem 4.2.1] (compare Corollary 3.16), the basis elements of $\theta^{*}\left(\mathbb{W}_{v}\left(-w_{0} \lambda\right)_{\mathbb{Z}}\right)$ afforded by the negative global basis, regarded as a subset of the $\widetilde{\mathfrak{g}}$-crystal $\mathbf{B}(\mathbb{X}(\lambda))$, decompose into the disjoint union of connected $\mathfrak{s l}(2, i)$-crystals (which is the set of labels of a basis of an irreducible $\mathfrak{s l}(2)$-module equipped with combinatorial operations $\widetilde{e}_{i}$ and $\widetilde{f_{i}}$ corresponding to $E_{i}^{(\bullet)}$ and $F_{i}^{(\bullet)}-$ see, e.g., [41, Definition 2.3.1]; it is the $i$-string in [42, 45]). Moreover, the basis elements of $\mathbb{W}_{s_{i} w w_{0}}(\lambda)_{\mathbb{Z}}$ afforded by the positive global basis, regarded as a subset of the $\widetilde{\mathfrak{g}}$-crystal $\mathbf{B}(\mathbb{X}(\lambda)$ ), also decompose into the disjoint union of $\mathfrak{s l}(2, i)$-crystals $([45,46])$, and the set $S$ of basis elements corresponding to each connected $\mathfrak{s l}(2, i)$-crystal satisfies

$$
S \cap \mathbb{W}_{w w_{0}}(\lambda)_{\mathbb{Z}}=S \text { or }\{\mathbf{v}\}
$$

where $\mathbf{v} \in S$ is the highest weight vector as a $\mathfrak{s l}(2, i)$-crystal, which has dominant weight as a weight of $\mathfrak{s l}(2, i)([45$, Lemma 2.6]). In particular, we have

$$
S \cap R_{w}^{v}(\mathrm{~J}, \lambda)^{\vee}=S \text { or }\{\mathbf{v}\} \text { or } \emptyset .
$$

Therefore, a reinterpretation of [42, (0.5)] using Theorem 4.33 (compare [39, 46]) reads as

$$
\begin{aligned}
\operatorname{ch} H^{0}\left(\mathcal{O}_{Q_{j}^{\prime}\left(v, s_{i} w\right)_{\mathbb{K}}}(\lambda)\right) & =\operatorname{ch} H^{0}\left(\mathcal{O}_{Q_{j}^{+}(v, w)_{\mathbb{K}}}(\lambda)\right) \\
0 & =\operatorname{ch} H^{1}\left(\mathcal{O}_{Q_{j}^{+}(v, w)_{\mathbb{K}}}(\lambda)\right)
\end{aligned}
$$

for each $\lambda \in P_{\mathrm{J},++}$. Since the zeroth part of the Demazure functor is the same as taking the maximal integrable $S L(2, i)$-inflation of a (weight-semisimple) $B_{i}$-module, we conclude that the inclusion $R_{w}^{v}(\mathrm{~J}, \lambda)_{\mathbb{K}}^{\vee} \subset R_{s_{i} w}^{v}(\mathrm{~J}, \lambda)_{\mathbb{K}}^{\vee}$ induces a natural inclusion

$$
H^{0}\left({Q_{J}^{\prime}}^{\prime}\left(v, s_{i} w\right)_{\mathbb{K}}, \mathcal{O}_{Q_{j}^{\prime}\left(\beta, s_{i} w\right)_{\mathbb{K}}}(\lambda)\right) \hookrightarrow H^{0}\left(Q_{\mathcal{J}}^{+}(v, w)_{\mathbb{K}}, \mathcal{O}_{Q_{j}^{+}(v, w)_{\mathbb{K}}}(\lambda)\right),
$$

which is an isomorphism by the character comparison. Thus, Lemma 4.29 implies

$$
\left(\pi_{i}\right)_{*} \mathcal{O}_{Q_{j}^{+}(v, w)_{\mathbb{K}}} \cong \mathcal{O}_{Q_{j}^{\prime}\left(v, s_{i} w\right)_{\mathbb{K}}} \text { and } \quad \mathbb{R}^{1}\left(\pi_{i}\right)_{*} \mathcal{O}_{Q_{J}^{+}(\beta, w)_{\mathbb{K}}} \cong\{0\} .
$$

Since we have $\mathbb{R}^{\geq 2}\left(\pi_{i}\right)_{*} \mathcal{O}_{Q_{J}^{+}}(v, w)_{\mathbb{K}} \cong\{0\}$ by dimension reasons, we conclude the result.

A particular case of Proposition 4.39 is worth noting:

Corollary 4.40. Set $\beta \in Q^{\vee}, w \in W$ and $\mathrm{J} \subset \mathrm{I}$. For each $i \in \mathrm{I}$ such that $s_{i} w<w$, we have a surjective map

$$
\pi_{i}: P_{i} \times{ }^{B} Q_{\jmath}^{\prime}(\beta, w)_{\mathbb{K}} \rightarrow Q_{\jmath}^{\prime}\left(\beta, s_{i} w\right)_{\mathbb{K}}
$$

such that $\mathbb{R}^{\bullet}\left(\pi_{i}\right)_{*} \mathcal{O}_{P_{i} \times{ }^{B} Q_{j}^{\prime}(\beta, w)_{\mathbb{K}}} \cong \mathcal{O}_{Q_{j}^{\prime}\left(\beta, s_{i} w\right)_{\mathbb{K}}}$.

\subsection{Lifting to and from characteristic 0}

Theorem 4.41. Let $\mathfrak{X}$ be a Noetherian scheme flat over $\mathbb{Z}$. If $\mathfrak{X}_{\mathbb{F}_{p}}$ is weakly normal for $p \gg 0$, then $\mathfrak{X}_{\mathbb{C}}$ is also weakly normal. 
Proof. Since the weak normalisation commutes with localisation [68, Theorem IV.3], we can argue locally. Let $(S, \mathfrak{m})$ be a local ring of $\mathfrak{X}_{\overline{\mathbb{Q}}}$ and let $S^{-}$be the weak normalisation of $S$ ([80, Remark 1]). By the Noetherian hypothesis, we can invert finitely many primes and take a finite algebraic extension of $\mathbb{Z}$ to obtain a ring $A$ such that we have a commutative $\operatorname{ring} S_{A}$ over $A$ and its ideal $\mathrm{m}_{A}$ with the following properties:

$\circ\left(S_{A} \otimes_{A} \overline{\mathbb{Q}}, \mathrm{m}_{A} \otimes_{A} \overline{\mathbb{Q}}\right) \cong(S, \mathfrak{m})$.

- The $A$-modules $S_{A}, \mathrm{~m}_{A}$ and $S_{A} / \mathrm{m}_{A}$ are torsion free.

- The specialisation of $A$ to the algebraic closure of a finite field yields a weakly normal (local) ring ([68, Theorem V.2]).

As $A$ is a Dedekind domain, we find that $S_{A}, \mathfrak{m}_{A}$ and $S_{A} / \mathfrak{m}_{A}$ are flat over $A$.

We have $S^{-}=S\left[f_{1}, \ldots, f_{n}\right]$, where $f_{1}, \ldots, f_{n}$ are integral elements. Multiplying with elements in $\overline{\mathbb{Q}}$, we can assume that $f_{1}, \ldots, f_{n}$ are integral over $S_{A}$. By inverting additional primes in $\mathbb{Z}$ if necessary (to assume that the denominator of $f_{i}$ in $\operatorname{Frac}(S)$ does not vanish along specialisations and achieve the conditions in the following), we can further assume that $S_{A}^{-}:=S_{A}\left[f_{1}, \ldots, f_{n}\right]$ is flat over $A$ and is integral for every specialisation of $A$ to a field.

The ring $\left(S_{A}^{-} / \mathfrak{m}_{A} S_{A}^{-}\right) \otimes_{A} \mathbb{C}$ is a finite-dimensional local commutative $\mathbb{C}$-algebra by the weak normality assumption on $S^{-}$[80, Remark 1]. In particular, the multiplication action of each element of $\left(S_{A}^{-} / \mathfrak{m}_{A} S_{A}^{-}\right) \otimes_{A} \mathbb{C}$ has a unique eigenvalue. Hence, if we present $\left(S_{A}^{-} / \mathfrak{m}_{A} S_{A}^{-}\right)=A\left[X_{1}, \ldots, X_{m}\right] / \sim$ (where $\sim$ contains the minimal polynomials of the $A$-valued matrix $X_{i}$ ), then the minimal polynomial of $X_{i}$ is of the form $\left(T-a_{i}\right)^{m_{i}}\left(a_{i} \in A\right)$. Therefore, we can assume that each $X_{i}$ is nilpotent by changing $X_{i}$ to $X_{i}+a_{i}$ if necessary. Hence, we conclude that $\left(X_{1}, \ldots, X_{m}\right) \subset\left(S_{A}^{-} / \mathrm{m}_{A} S_{A}^{-}\right)$have eigenvalues 0 after specialising to $\overline{\mathbb{F}}_{p}$.

By assumption, $\left(S_{A}\right) \otimes_{A} \overline{\mathbb{F}}_{p}$ is weakly normal for every possible prime $p$ and every ring homomorphism $A \rightarrow \overline{\mathbb{F}}_{p}$. Hence, the specialisation $\left(S_{A}^{-} / \mathrm{m}_{A} S_{A}^{-}\right) \otimes_{A} \overline{\mathbb{F}}_{p}$ must contain $\overline{\mathbb{F}}_{p}$ as its ring direct summand (if it is nonzero). This forces rank $\left(S_{A}^{-} / \mathrm{m}_{A} S_{A}^{-}\right)=1$, since we cannot have two linearly independent idempotents that have distinct eigenspaces, by the previous paragraph.

Therefore, we deduce that $S_{A}^{-}=S_{A}$, which implies that $(S, \mathfrak{m})$ is itself weakly normal. In view of [68, Theorem V.2 and Corollary V.3], we conclude the assertion.

Corollary 4.42. For each $\mathrm{J} \subset \mathrm{I}, w, v \in W_{\mathrm{af}}$, the scheme $\mathbb{Q}_{\mathrm{J}}^{\prime}(v, w)_{\mathbb{C}}$ is weakly normal.

Proof. Apply Theorem 4.41 to Lemma 4.7.

Proposition 4.43. For each $\mathrm{J} \subset \mathrm{I}, v, w \in W$, the variety ${Q_{\mathrm{J}}^{\prime}}(v, w)_{\overline{\mathbb{F}}_{p}}$ is normal for $p \gg 0$, provided $\mathbb{Q}_{\mathrm{J}}^{\prime}(v, w)_{\mathbb{C}}$ is normal. The same is true for irreducibility.

Proof. Since $\mathscr{Q}_{j}^{\prime}(v, w)$ is defined over $\mathbb{Z}$, the scheme $\mathscr{Q}_{\jmath}^{\prime}(v, w)_{\mathbb{C}}$ is a scalar extension of $\mathbb{Q}_{\jmath}^{\prime}(v, w)_{\overline{\mathbb{Q}}}$. By [77, Lemma 038P], we deduce that $\widehat{Q}_{j}^{\prime}(v, w)_{\bar{Q}}$ is normal. We apply [77, Lemma 0364] to derive the irreducibility of $\mathbb{Q}_{J}^{\prime}(v, w)_{\bar{Q}}$. Now apply [36, Proposition 9.9.4 and Théorème 9.7.7] to $\mathbb{Q}_{J}^{\prime}(v, w)_{\mathbb{Z}} \rightarrow$ $\operatorname{Spec} \mathbb{Z}$.

\section{Normality of quasi-map spaces}

In this section, we continue to work in the setting of the previous section, with the exception that $\mathbb{K}=\mathbb{C}$. Also, a point of a scheme (over $\mathbb{C}$ ) means a closed point unless stated otherwise.

\subsection{Graph-space resolution of $\mathbb{Q}(\beta)$}

We refer to [56, 30, 6, 23, 33] for precise explanations of the material in this subsection. For each nonnegative integer $n$ and $\beta \in Q_{+}^{\vee}$, we set $\mathscr{G}_{\mathscr{B}_{n, \beta}}$ to be the space of stable maps of genus 0 curves with $n$-parked points to $\left(\mathbb{P}^{1} \times \mathscr{B}\right)$ of bidegree $(1, \beta)$, which is also called the graph space of $\mathscr{B}$. A point 
of $\mathscr{G} \mathscr{B}_{n, \beta}$ represents a genus 0 curve $C$ with $n$-marked points, together with a map to $\mathbb{P}^{1}$ of degree 1 (obtained by composing the map from $C$ to $\mathbb{P}^{1} \times \mathscr{B}$ with the first projection of the target). Hence, we have a unique $\mathbb{P}^{1}$-component of $C$ that maps isomorphically onto $\mathbb{P}^{1}$. We call this component the main component of $C$ and denote it by $C_{0}$. The space $\mathscr{G}_{\mathscr{B}_{n, \beta}}$ is a normal projective variety by [30, Theorem 2], which has at worst quotient singularities arising from the automorphism of curves (and hence is smooth as an orbifold). The natural $\left(H \times \mathbb{G}_{m}\right)$-action on $\left(\mathbb{P}^{1} \times \mathscr{B}\right)$ induces a natural $\left(H \times \mathbb{G}_{m}\right)$-action on $\mathscr{G}_{n, \beta}$.

We have a morphism $\pi_{n, \beta}: \mathscr{G}_{\mathscr{B}_{n, \beta}} \rightarrow \mathscr{Q}(\beta)$ that factors through $\mathscr{G}_{\mathscr{B}_{0, \beta}}$ (Givental's main lemma [34]; see [23, §8.3]). Let $\mathrm{e}_{j}: \mathscr{B}_{n, \beta} \rightarrow \mathscr{B}(1 \leq j \leq n)$ be the evaluation at the $j$ th marked point, and let $\mathrm{ev}_{j}: \mathscr{G} \mathscr{B}_{n, \beta} \rightarrow \mathscr{B}$ be the $j$ th evaluation map to $\mathbb{P}^{1} \times \mathscr{B}$ composed with the second projection.

Since $\mathbb{Q}(\beta)$ is irreducible (Theorem 2.7), [23, §8.3] asserts that $\mathscr{G} \mathscr{B}_{n, \beta}$ is irreducible (as a special feature of flag varieties; see [30, §1.2] and [53]).

\subsection{The variety $Q(\beta, v, w)$}

Let $\mathscr{G} \mathscr{B}_{2, \beta}^{\mathrm{b}}$ denote the subvariety of $\mathscr{G} \mathscr{B}_{2, \beta}$ consisting of points such that the first marked point projects to $0 \in \mathbb{P}^{1}$ and the second projects to $\infty \in \mathbb{P}^{1}$ through the projection of quasi-stable curves $C$ to their main component $C_{0} \cong \mathbb{P}^{1}$. Let us denote the restrictions of $\operatorname{ev}_{i}(i=1,2)$ and $\pi_{2, \beta}$ to $\mathscr{G}_{2, \beta}^{\mathrm{b}}$ by the same letter. By $[9,10], \mathscr{G} \mathscr{B}_{2, \beta}^{\mathrm{b}}$ gives a resolution of singularities of $\mathcal{Q}(\beta)$ (in an orbifold sense).

Recall that each Schubert cell $\mathbb{O}_{\mathscr{B}}(w)$ contains a unique $H$-fixed point $p_{w}$. For each $w \in W$, we set

$$
\mathbb{O}_{\mathscr{B}}^{\mathrm{op}}(w):=N^{-} p_{w} \subset \mathscr{B}, \quad \mathscr{B}^{\mathrm{op}}(w):=\overline{\mathbb{O}_{\mathscr{B}}^{\mathrm{op}}(w)}=\overline{N^{-} p_{w}} \subset \mathscr{B} .
$$

For $w, v \in W$, we define

$$
\mathscr{G} \mathscr{B}_{2, \beta}^{\mathrm{b}}(w, v):=\operatorname{ev}_{1}^{-1}(\mathscr{B}(w)) \cap \mathrm{ev}_{2}^{-1}\left(\mathscr{B}^{\mathrm{op}}(v)\right) \subset \mathscr{G}_{2, \beta}^{\mathrm{b}}
$$

and

$$
\mathscr{B}_{2, \beta}(w, v):=\mathrm{e}_{1}^{-1}(\mathscr{B}(w)) \cap \mathrm{e}_{2}^{-1}\left(\mathscr{B}^{\mathrm{op}}(v)\right) \subset \mathscr{B}_{2, \beta} .
$$

Theorem 5.1 ([16]). For each $v, w \in W$ and $\beta \in Q_{+}^{\vee}$, the variety $\mathscr{G} \mathscr{B}_{2, \beta}^{\mathrm{b}}(w, v)$ is either empty or a unirational (and hence connected and irreducible) variety that has rational singularities. The same is true for $\mathscr{B}_{2, \beta}(w, v)$. In particular, they are normal.

Proof. Since both cases are parallel, we concentrate on the case of $\mathscr{G}_{2, \beta}^{b}(w, v)$.

As $\mathbb{P}^{1} \times \mathscr{B}$ is a homogeneous variety under the group action of $(S L(2) \times G)$, [53, Theorem 2 and Theorem 3] apply and hence $\mathscr{G}_{2, \beta}$ is a rational variety. Then a pair of Schubert subvarieties (with respect to a pair of opposite Borel subgroups of $S L(2) \times G)$ of $\mathbb{P}^{1} \times \mathscr{B}$ presented as $\{0\} \times \mathscr{B}(w)$ and $\{\infty\} \times \mathscr{B}^{\mathrm{op}}(v)$ is used to define $\mathscr{G} \mathscr{B}_{2, \beta}^{\mathrm{b}}(w, v)$. Hence, [16, Proposition $\left.3.2 \mathrm{c}\right)$ ] implies that $\mathscr{G} \mathscr{B}_{2, \beta}^{\mathrm{b}}(w, v)$ is either empty or unirational (and hence connected). Since a pair of Schubert varieties with respect to the opposite Borel subgroups forms the dense subset of the pair of translations of Schubert varieties by applying the $G$-action, it must contain a pair of Schubert varieties in general position. Therefore, [16, Corollary 3.1] implies that $\mathscr{G} \mathscr{B}_{2, \beta}^{b}(w, v)$ has rational singularity. The last assertion is a well-known property of rational singularities [55, Definition 5.8].

Corollary 5.2. In the setting of Theorem 5.1, we additionally assume that $\mathscr{G}_{2, \beta}^{b}(w, v)$ is nonempty. Then we have

$$
\operatorname{dim} \mathscr{G} \mathscr{B}_{2, \beta}^{\mathrm{b}}(w, v)=\operatorname{dim} \mathscr{Q}(\beta)-\ell(w)-\ell\left(v w_{0}\right) .
$$

Proof. By [16, Proposition 3.2 b)], the dimension count of (a Zariski open subset of) $\mathscr{G} \mathscr{B}_{2, \beta}^{b}(w, v)$ can be borrowed from [54, Theorem 2] applied to the $(S L(2) \times G)$-action on $\mathbb{P}^{1} \times \mathscr{B}$. In addition, the map 
$\pi_{2, \beta}$ is birational. Therefore, we have

$$
\begin{aligned}
\operatorname{dim} \mathscr{G}_{2, \beta}^{\mathrm{b}}(w, v) & =\operatorname{dim} Q(\beta)-\operatorname{codim}_{\mathscr{B}} \mathscr{B}(w)-\operatorname{codim}_{\mathscr{B}} \mathscr{B}^{\mathrm{op}}(v) \\
& =\operatorname{dim} Q(\beta)-\ell(w)-\ell\left(v w_{0}\right),
\end{aligned}
$$

as required.

Proposition 5.3. For each $v, w \in W$ and $\beta \in Q_{+}^{\vee}$, the variety $Q^{\prime}\left(v t_{\beta}, w\right)_{\mathbb{C}}$ is irreducible.

Proof. We define

$$
\stackrel{Q}{Q}^{\prime}\left(v t_{\beta}, w\right)_{\mathbb{C}}:=\mathscr{Q}^{\prime}\left(v t_{\beta}, w\right)_{\mathbb{C}} \backslash\left(\left(\bigcup_{w^{\prime}<\frac{\infty}{2} w} Q^{\prime}\left(v t_{\beta}, w^{\prime}\right)_{\mathbb{C}}\right) \cup \underset{v^{\prime}>\frac{\infty}{2} v}{\bigcup} Q^{\prime}\left(v^{\prime} t_{\beta}, w\right)_{\mathbb{C}}\right) .
$$

In view of Corollary 4.31, every irreducible component of ${Q^{\prime}}^{\prime}\left(v t_{\beta}, w\right)_{\mathbb{C}}$ has dimension $\ell^{\frac{\infty}{2}}\left(v t_{\beta}\right)-\ell^{\frac{\infty}{2}}(w)$ if it is nonempty. We have $\mathbb{Q}^{\prime}\left(v t_{\beta}, w\right)_{\mathbb{C}} \neq \emptyset$ if and only if $v t_{\beta} \leq \frac{\infty}{2} w$, by Corollary 4.21. In particular, we find that $\stackrel{\circ}{Q}^{\prime}\left(v t_{\beta}, w\right)_{\mathbb{C}} \neq \emptyset$ if $v t_{\beta} \leq \frac{\infty}{2} w$, and in that case all of its irreducible components have dimension

$$
\ell^{\frac{\infty}{2}}\left(v t_{\beta}\right)-\ell^{\frac{\infty}{2}}(w)=\ell(v)-\ell(w)+2\langle\beta, \rho\rangle .
$$

A point of $\stackrel{Q}{Q}^{\prime}\left(v t_{\beta}, w\right)_{\mathbb{C}}$ can be seen as a quasi-map of degree $\beta$ with no defects at 0 and $\infty$ (Theorem 4.30). In particular, $\mathscr{G}_{2, \beta}^{b}(w, v) \neq \emptyset$ if $\mathscr{Q}^{\prime}\left(v t_{\beta}, w\right)_{\mathbb{C}} \neq \emptyset$. When $\mathscr{Q}^{\prime}\left(v t_{\beta}, w\right)_{\mathbb{C}} \neq \emptyset$, we have

$$
\begin{aligned}
\operatorname{dim} \mathscr{G} \mathscr{B}_{2, \beta}^{b}(w, v) & =\operatorname{dim} \mathscr{Q}(\beta)-\ell(w)-\ell\left(v w_{0}\right) \\
& =2\langle\beta, \rho\rangle+\operatorname{dim} \mathscr{B}-\ell\left(w_{0}\right)+\ell(v)-\ell(w)=(5.1),
\end{aligned}
$$

where the first equality follows from Corollary 5.2 and the second follows from Theorem 2.7. By the surjectivity of $\pi_{2, \beta}$ and equation (5.2), we find that the rational map $\mathscr{G}_{\mathscr{B}_{2, \beta}^{b}}^{b}(w, v) \rightarrow \mathscr{Q}^{\prime}\left(v t_{\beta}, w\right)_{\mathbb{C}}$ is surjective and generically finite (it is in fact birational, by Theorem 5.7 and Proposition 5.11). In view of Theorem 5.1, the variety $\mathscr{G} \mathscr{B}_{2, \beta}^{b}(w, v)$ is irreducible if it is nonempty. Therefore, $\mathscr{Q}^{\prime}\left(v t_{\beta}, w\right)_{\mathbb{C}} \neq \emptyset$ implies that $\stackrel{\circ}{Q}^{\prime}\left(v t_{\beta}, w\right)_{\mathbb{C}}$ is irreducible. Again by Corollary 4.31 , we conclude that $\mathbb{Q}^{\prime}\left(v t_{\beta}, w\right)_{\mathbb{C}}$ has a unique irreducible component, as required.

In view of Proposition 5.3, we set $\mathcal{Q}_{\mathrm{J}}(\beta, v, w):=\mathscr{Q}_{\mathrm{J}}^{\prime}\left(v t_{\beta}, w\right)_{\mathbb{C}}$ for each $\mathrm{J} \subset \mathrm{I}, \beta \in Q_{+}^{\vee}$ and $v, w \in W$ in what follows (see also Corollary 5.21). We have $Q\left(\beta, w_{0}, w\right)=\mathscr{Q}(\beta, w)$ by Theorem 4.30.

Corollary 5.4 (Corollary of the proof of Proposition 5.3). For each $\beta \in Q_{+}^{\vee}$ and $v, w \in W$, the scheme $\mathbb{Q}(\beta, v, w)$ is nonempty if and only if $v t_{\beta} \leq_{\frac{\infty}{2}} w$. If it is nonempty, then it has dimension $\ell(v)-\ell(w)+2\langle\beta, \rho\rangle$. Moreover, the space $\mathbb{Q}(\beta, v, w)$ (viewed as a subspace of $\mathbb{Q}(\beta)$ ) contains a quasi-map with no defects at 0 and $\infty$.

Remark 5.5. Corollary 5.4 removes the condition $\gamma \gg 0$ from [29, Lemma 8.5.2].

Thanks to Proposition 5.3 and its proof, we deduce that the map $\pi_{2, \beta}$ restricts to an $\left(H \times \mathbb{G}_{m}\right)$ equivariant birational proper map

$$
\pi_{\beta, w, v}: \mathscr{G}_{2, \beta}^{\mathrm{b}}(w, v) \rightarrow Q(\beta, v, w),
$$

by inspection. 


\subsection{From Givental's main lemma}

For each $w, v \in W$, we define subvarieties of $\mathscr{B}_{2, \beta}$ as

$$
\mathscr{B}_{2, \beta}[w]:=\mathrm{e}_{1}^{-1}\left(p_{w}\right), \quad \mathscr{B}_{2, \beta}[w, v]:=\mathrm{e}_{1}^{-1}\left(p_{w}\right) \cap \mathrm{e}_{2}^{-1}\left(p_{v}\right) .
$$

Similarly, we set

$$
\mathscr{G} \mathscr{B}_{2, \beta}^{\mathrm{b}}[w, v]:=\operatorname{ev}_{1}^{-1}\left(p_{w}\right) \cap \mathrm{ev}_{2}^{-1}\left(p_{v}\right) \subset \mathscr{G}_{\mathscr{B}_{2, \beta}^{b}}^{\mathrm{b}}, \quad \mathscr{B}_{1, \beta}[w]:=\mathrm{e}_{1}^{-1}\left(p_{w}\right) \subset \mathscr{B}_{1, \beta}[w] .
$$

Lemma 5.6. For each $x, y \in \mathscr{B}$ and $\beta \in Q_{+}^{\vee}$, there exists $w \in W$ such that

$$
\mathrm{e}_{1}^{-1}(x) \cap \mathrm{e}_{2}^{-1}(y) \cong \mathscr{B}_{2, \beta}\left[w, w_{0}\right]
$$

The same is true for ev and $\mathscr{G} \mathscr{B}$.

Proof. We consider only the case of e and $\mathscr{B}$, as the other case is completely parallel. Since $(x, y) \in$ $\mathscr{B} \times \mathscr{B}$ and the $G$-action on $\mathscr{B}$ is transitive, we can assume $y=p_{w_{0}}$. Since we have $\operatorname{Stab}_{G} y=B$, we can further rearrange $x=p_{w}$ for some $w \in W$ by equation (2.4).

Theorem 5.7 (Givental's main lemma [34]; see [23]). Set $\beta \in Q_{+}^{\vee}$. Let $(f, D) \in \mathbb{Q}(\beta)$ be a quasi-map with defect $D=\sum_{x \in \mathbb{P}^{1}(\mathbb{C})} \beta_{x} \otimes[x]$. Then we have

$$
\pi_{2, \beta}^{-1}(f, D) \cong \mathscr{B}_{2, \beta_{0}}[w] \times \mathscr{B}_{2, \beta_{\infty}}[w] \times \prod_{x \in \mathbb{P}^{1}(\mathbb{C}) \backslash\{0, \infty\}} \mathscr{B}_{1, \beta_{x}}[w] \subset \mathscr{G} \mathscr{B}_{2, \beta}^{b} .
$$

In particular, the map $\pi_{2, \beta}$ is an isomorphism along the locus with $D=0$.

Remark 5.8. In Theorem 5.7, the first marked points of (the stable maps in) $\mathscr{G} \mathscr{B}_{2, \beta}^{b}$ (the marked points at $0 \in \mathbb{P}^{1}$; i.e., the image of $\mathrm{ev}_{1}$ ) are identified with the second marked points of (the stable maps in) $\mathscr{B}_{2, \beta_{0}}[w]$ (i.e., the image of $\mathrm{e}_{2}$ ), and the second marked points of $\mathscr{G}_{2, \beta}^{\mathrm{b}}$ (the marked points at $\infty \in \mathbb{P}^{1}$ ) are identified with the second marked points of $\mathscr{B}_{2, \beta_{\infty}}[w]$. (Other marked points are used to glue pieces of stable maps together.)

Lemma 5.9 ([29]). For each $\beta \in Q_{+}^{\vee}$ such that $\left\langle\beta, \alpha_{i}\right\rangle \geq 1$ for all $i \in \mathrm{I}$, the evaluation map

$$
\mathrm{ev}:=\left(\mathrm{ev}_{1} \times \mathrm{ev}_{2}\right): \mathscr{G} \mathscr{B}_{2, \beta}^{\mathrm{b}} \longrightarrow \mathscr{B} \times \mathscr{B}
$$

is surjective.

Proof. Taking into account the fact that $\mathscr{G}_{2, \beta}^{b}$ is projective, it suffices to prove that the tangent map associated to ev is surjective on a dense open subset of $\mathscr{G} \mathscr{B}_{2, \beta}^{b}$.

Since the map $\pi_{2, \beta}$ is birational by Theorem 5.7, we replace the problem with the case of a genuine map $f: \mathbb{P}^{1} \rightarrow \mathscr{B}$. Thanks to [29, Proposition 3.5], $Q(\beta)$ and hence $\mathscr{G} \mathscr{B}_{2, \beta}^{b}$ are smooth at $f$. Moreover, its tangent space is described as

$$
H^{0}\left(\mathbb{P}^{1}, f^{*} T \mathscr{B}\right), \quad H^{1}\left(\mathbb{P}^{1}, f^{*} T \mathscr{B}\right)=\{0\},
$$

and the filtration of $T \mathscr{B}$ as $G$-equivariant line bundles yields the associated graded

$$
\bigoplus_{\alpha \in \Delta_{+}} H^{0}\left(\mathbb{P}^{1}, \mathcal{O}_{\mathbb{P}^{1}}(\langle\beta, \alpha\rangle)\right)
$$

(Here we used $\left\langle\beta, \alpha_{i}\right\rangle \geq-1$ for each $i \in \mathrm{I}$.) In particular, we have

$$
\operatorname{dim} H^{0}\left(\mathbb{P}^{1}, f^{*} T \mathscr{B}\right)=\Sigma_{\alpha \in \Delta_{+}} \operatorname{dim} H^{0}\left(\mathbb{P}^{1}, \mathcal{O}_{\mathbb{P}^{1}}(\langle\beta, \alpha\rangle)\right) .
$$


The effect of fixing the image of two points $0, \infty \in \mathbb{P}^{1}$ corresponds to imposing a divisor twist by $\mathcal{O}_{\mathbb{P}^{1}}(-[0]-[\infty])$. We have

$$
\begin{aligned}
\operatorname{dim} H^{0}\left(\mathbb{P}^{1}, f^{*} T \mathscr{B} \otimes_{\mathcal{O}_{\mathbb{P}^{1}}} \mathcal{O}_{\mathbb{P}^{1}}(-[0]-[\infty])\right) & \leq \operatorname{dim} \bigoplus_{\alpha \in \Delta_{+}} H^{0}\left(\mathbb{P}^{1}, \mathcal{O}_{\mathbb{P}^{1}}(\langle\beta, \alpha\rangle-2)\right) \\
& =\operatorname{dim} \bigoplus_{\alpha \in \Delta_{+}} H^{0}\left(\mathbb{P}^{1}, \mathcal{O}_{\mathbb{P}^{1}}(\langle\beta, \alpha\rangle)\right)-2\left|\Delta_{+}\right| \\
& =\operatorname{dim} \mathcal{Q}(\beta)-2 \operatorname{dim} \mathscr{B} \\
& =\operatorname{dim} \mathscr{G} \mathscr{B}_{2, \beta}^{\mathrm{b}}-2 \operatorname{dim} \mathscr{B} .
\end{aligned}
$$

Here the first inequality comes from the short exact sequences, the equality that follows is $\left\langle\beta, \alpha_{i}\right\rangle \geq 1$ for each $i \in \mathrm{I}$, the next equality is the smoothness of $\mathscr{Q}(\beta)$ at $f$ and $\left|\Delta_{+}\right|=\operatorname{dim} \mathscr{B}$ and the final equality is the birationality of $\pi_{2, \beta}$ (restricted to $\mathscr{G}_{2, \beta}^{\mathrm{b}} \subset \mathscr{G}_{2, \beta}$ ).

From this, we deduce that $d \pi_{2, \beta}$ is generically surjective, as required.

Proposition 5.10. For each $\beta \in Q_{+}^{\vee}$ such that $\left\langle\beta, \alpha_{i}\right\rangle \geq 1$ for all $i \in \mathrm{I}$ and $w, v \in W$, the scheme $\mathscr{G} \mathscr{B}_{2, \beta}^{\mathrm{b}}[w, v]$ is connected and nonempty.

Proof. The map ev borrowed from Lemma 5.9 is $G$-equivariant, and $(\mathscr{B} \times \mathscr{B})$ admits an open dense $G$-orbit $\mathbf{O}$ by the Bruhat decomposition. Since $\mathscr{G} \mathscr{B}_{2, \beta}^{\mathrm{b}}$ is an irreducible variety, so is its Zariski open set $\mathrm{ev}^{-1}(\mathbf{O})$. If we consider $x \in \mathbf{O}$, then the irreducible components $\mathrm{of}^{-1}(x)$ (which must be finite, as we consider varieties that are finite types) must be permuted by $\operatorname{Stab}_{G} x \cong H$. Since $H$ is connected, we cannot have a nontrivial action. Therefore, the irreducible component of $\mathrm{ev}^{-1}(x)$ must be unique. Thanks to the Stein factorisation theorem [77, Theorem 03H0], the map ev factors through the normalisation $Y$ of $(\mathscr{B} \times \mathscr{B})$ inside (the function field of) $\mathscr{G} \mathscr{B}_{2, \beta}^{\mathrm{b}}$, and the map $\mathscr{G} \mathscr{B}_{2, \beta}^{\mathrm{b}} \rightarrow Y$ has connected fibre. Since the general fibre of ev is connected, so is the map $Y \rightarrow(\mathscr{B} \times \mathscr{B})$. It follows that $Y \rightarrow(\mathscr{B} \times \mathscr{B})$ is a birational map (which is also finite, by the Stein factorisation theorem). This implies that $Y$ and $(\mathscr{B} \times \mathscr{B})$ share the same function field. Thus, we conclude that $Y \cong(\mathscr{B} \times \mathscr{B})$ by the normality of $(\mathscr{B} \times \mathscr{B})$. This particularly means that every fibre of ev is connected. Therefore, the assertion follows by choosing $(H \times H)$-fixed points in $(\mathscr{B} \times \mathscr{B})$ as particular cases.

Proposition 5.11. For each $\beta \in Q_{+}^{\vee}$ such that $\left\langle\beta, \alpha_{i}\right\rangle \geq 1$ for all $i \in \mathrm{I}$ and $w, v \in W$, the scheme $\mathscr{B}_{2, \beta}[w, v]$ is connected and nonempty. For each $\beta \in Q_{+}^{\vee}$ and $w \in W$, the schemes $\mathscr{B}_{1, \beta}[w]$ and $\mathscr{B}_{2, \beta}[w]$ are connected and nonempty.

Proof. We prove the first assertion. We have a rational map $\mathscr{G}_{\mathscr{B}_{2, \beta}}^{b}[w, v] \rightarrow \mathscr{B}_{2, \beta}[w, v]$ obtained by forgetting the map to $\mathbb{P}^{1}$. Moreover, the locus of this map is not defined consists of stable maps whose main components have degree 0 and have only two marked points. By modifying the universal family by adjoining two such marked points in such a degree 0 component, we conclude that $\mathscr{G} \mathscr{B}_{2, \beta}^{b}[w, v] \rightarrow$ $\mathscr{B}_{2, \beta}[w, v]$ extends to a map of topological spaces. By examining the condition to be a stable map $[30, \S 1.1]$, we deduce that this map is surjective onto the image. Therefore, the connectedness and nonemptiness of $\mathscr{G}_{2, \beta}^{\mathrm{b}}[w, v]$ imply that $\mathscr{B}_{2, \beta}[w, v]$ is likewise connected and nonempty - which is the first assertion.

The second assertion is straightforward from the irreducibility of $\mathscr{B}_{n, \beta}$, together with the fact that $\mathrm{e}_{1}$ is a $G$-equivariant fibre bundle over $\mathscr{B}$ (see the proof of Proposition 5.10).

Lemma 5.12. Set $\beta \in Q_{+}^{\vee}$. Let $(f, D) \in \mathbb{Q}(\beta)$ be a quasi-map with defect $D$ with the following properties:

○ $D=\sum_{x \in \mathbb{P}^{1}(\mathbb{C})} \beta_{x} \otimes[x]$.

$\circ\left\langle\beta_{0}, \alpha_{i}\right\rangle \geq 1$ and $\left\langle\beta_{\infty}, \alpha_{i}\right\rangle \geq 1$ for each $i \in \mathrm{I}$.

Then $\pi_{\beta, w, v}^{-1}(f, D)$ is connected for every $w, v \in W$ if it is nonempty. 
Proof. By Proposition 5.11 and Theorem 5.7, we can forget about the contribution of $\mathscr{B}_{1, \beta_{x}}[w]$ when $x \neq 0, \infty$. By our assumption and Proposition 5.11, we know that

$$
\mathscr{B}_{2, \beta_{0}}\left[u, w^{\prime}\right] \neq \emptyset, \quad \mathscr{B}_{2, \beta_{\infty}}\left[u, v^{\prime}\right] \neq \emptyset,
$$

which are nonempty and connected for each $u, w^{\prime}, v^{\prime} \in W$. Since $\mathscr{B}_{2, \beta_{0}}$ and $\mathscr{B}_{2, \beta_{\infty}}$ are proper, we always find a limit point with respect to the $H$-action. It follows that $\mathscr{B}_{2, \beta_{0}}[u] \cap \mathrm{e}_{2}^{-1}\left(\mathscr{B}\left(u^{\prime}\right)\right.$ ) (resp., $\left.\mathscr{B}_{2, \beta_{\infty}}[u] \cap \mathrm{e}_{2}^{-1}\left(\mathscr{B}^{\mathrm{op}}\left(u^{\prime}\right)\right)\right)$ is connected for each $u, u^{\prime} \in W$, as we can connect every two points by appropriately sending to or from $H$-limit points that are contained in a connected component of the form $\mathscr{B}_{2, \beta_{0}}\left[u, w^{\prime}\right]\left(w^{\prime} \in W\right)$. Thanks to Theorem 5.7, we conclude the assertion.

\subsection{Normality of $\mathbb{Q}(\beta, v, w)$}

Let $\mathscr{Q}^{+}(\beta, v, w)$ be the normalisation of $Q(\beta, v, w)$ for each $\beta \in Q_{+}^{\vee}$ and $v, w \in W$. We denote the normalisation map by $\eta_{\beta, v, w}: \mathbb{Q}^{+}(\beta, v, w) \rightarrow \mathbb{Q}(\beta, v, w)$.

Proposition 5.13. For each $\beta \in Q_{+}^{\vee}$ and $w, v \in W$, the variety $Q(\beta, v, w)$ is normal if and only if every fibre of $\pi_{\beta, v, w}$ is connected.

Proof. As $\mathscr{G}_{2, \beta}^{b}(v, w)$ is normal and $\pi_{\beta, v, w}$ is proper, we know that

$$
\left(\pi_{\beta, v, w}\right)_{*} \mathcal{O}_{\mathscr{G} \mathscr{B}_{2, \beta}^{b}(v, w)} \cong \mathcal{O}_{Q^{+}(\beta, v, w)} .
$$

The properness of $\pi_{\beta, v, w}$ also implies that $\mathcal{O}_{Q^{+}(\beta, v, w)}$ is a coherent sheaf on $\mathbb{Q}(\beta, v, w)$. For each closed point $x$ of $Q(\beta, v, w)$, we set

$$
\Theta(x):=\operatorname{dim}_{\mathbb{C}} \mathcal{O}_{Q^{+}(\beta, v, w)} \otimes_{\mathcal{O}_{Q}(\beta, v, w)} \mathbb{C}_{x} .
$$

By the Stein factorisation theorem, the map $\eta_{\beta, v, w}$ is finite. By Corollary 4.42 and Section 5.2, we know that the variety $\mathbb{Q}(\beta, v, w)$ is weakly normal. From this, we deduce that $\Theta(x)=\# \eta_{\beta, v, w}^{-1}(x)$ (compare [80, Remark 1]). Moreover, it counts the number of irreducible components of the fibre of $\eta_{\beta, v, w}$.

The coherence of $\mathcal{O}_{Q^{+}}(\beta, v, w)$ implies that the right-hand side of formula (5.3) is an uppersemicontinuous function on $Q(\beta, v, w)$, and hence so is $\Theta$.

If we have $\Theta \equiv 1$ on $Q(\beta, v, w)$, then we have $\widehat{Q}^{+}(\beta, v, w)=\mathbb{Q}(\beta, v, w)$ by the weak normality of the latter (compare [80, Remark 1]). Therefore, the 'if' part of the assertion follows.

If we have $\Theta \neq \equiv 1$ on $\mathbb{Q}(\beta, v, w)$, then we have $\mathbb{Q}^{+}(\beta, v, w) \neq \mathbb{Q}(\beta, v, w)$. Hence, the 'only if' part of the assertion follows.

These complete the proof of the proposition.

Corollary 5.14. (Braverman and Finkelberg). For each $\beta \in Q_{+}^{\vee}$, the variety $Q(\beta)$ is normal.

Remark 5.15. Our proof of Corollary 5.14 is independent of [9] (although based on common earlier papers $[29,23])$. Hence, we obtain a new proof of the normality of $\mathscr{Q}(\beta)$ and $\mathscr{Z}\left(\beta, w_{0}\right)$. Together with Theorem 4.33, Corollary 5.14 also makes the contents of [51] logically independent of [9] (compare Appendix A).

Proof of Corollary 5.14. Recall that $Q(\beta)=\mathscr{Q}\left(\beta, w_{0}, e\right)$. We borrow the upper-semicontinuous function $\Theta$ that counts the number of connected components of the fibre of $\eta_{\beta, w_{0}, e}$ from formula (5.3) in the proof of Proposition 5.13.

By Proposition 5.13, it suffices to prove that $\Theta \equiv 1$ on $Q(\beta)$. In other words, it suffices to prove that the fibre $\eta_{\beta, w_{0}, e}^{-1}(x)$ is connected for each $x \in \mathbb{Q}(\beta)$.

By Theorem 5.7, we deduce that the set of connected components of $\eta_{\beta, w_{0}, e}^{-1}(x)$ is in bijection with the set of connected components of $\prod_{y \in \mathbb{P}^{1}(\mathbb{C})} \mathscr{B}_{\beta_{y}}[w]$. By Proposition 5.11, this latter space is connected.

Therefore, we conclude the result. 
Theorem 5.16. For each $\beta \in Q_{+}^{\vee}$ and $w \in W$, the varieties $\mathbb{Q}\left(\beta, w_{0}, w_{0}\right)$ and $\mathbb{Q}\left(\beta, e, w_{0}\right)$ are normal.

Proof. We set $(v, w)=\left(w_{0}, w_{0}\right)$ or $\left(e, w_{0}\right)$. We borrow the upper-semicontinuous function $\Theta$ that counts the number of connected components of the fibre of $\eta_{\beta, v, w}$ from formula (5.3) in the proof of Proposition 5.13.

By Proposition 5.13, it suffices to prove that $\Theta \equiv 1$ on $Q(\beta, v, w)$ by assuming the contrary to deduce a contradiction. For each $x \in \mathbb{Q}(\beta, v, w)$ such that $\Theta(x) \geq 2$, the fibre $\eta_{\beta, w_{0}, e}^{-1}(x)$ is disconnected.

By our choice of $(v, w)$ and Theorem 5.7 (compare Proposition 5.11 and Corollary 5.14), we deduce that the set of connected components of $\eta_{\beta, v, w}^{-1}(x)$ is in bijection with the set of connected components of $\mathscr{B}_{2, \beta_{0}}\left[u, w_{0}\right]$ or $\mathscr{B}_{2, \beta_{0}}\left[u, w_{0}\right] \times \mathscr{B}_{2, \beta_{\infty}}\left[u^{\prime}, w_{0}\right]$ for some $u, u^{\prime} \in W$.

To see whether this is the case, we specialise to the case of $(v, w)=\left(w_{0}, w_{0}\right)$ (to guarantee that the contribution at $\infty \in \mathbb{P}^{1}$ in Theorem 5.7 is the same as the points in $\mathbb{C}^{\times} \subset \mathbb{P}^{1}$, which are connected by Proposition 5.11). We can choose $\beta_{0}<\beta_{0}^{\prime} \in Q_{+}^{\vee}$ such that $\left\langle\alpha_{i}^{\vee}, \beta_{0}^{\prime}\right\rangle \geq 1$ for every $i \in \mathrm{I}$. By Proposition 5.11 , we deduce that the fibre of the locus $\mathscr{Z}$ of $\mathscr{Q}\left(\beta, w_{0}\right)$ whose points have defect $\beta_{0}^{\prime}$ along 0 is connected (if it is nonempty) for every $\beta \in Q^{\vee}$. Hence we have $\Theta(y)=1$ for each $y \in \mathscr{Z}$.

For each $\tilde{x} \in \mathscr{G}_{2}^{b}$ such that $x=\pi_{2, \beta}(\tilde{x}) \in \mathbb{Q}\left(\beta, w_{0}\right)$ has defect $\beta_{0}$ at 0 , we can replace $\beta$ with $\beta+\beta_{0}^{\prime}-\beta_{0}$ and add additional irreducible components $C^{\prime}$ to the main component $\mathbb{P}^{1}$ of $\tilde{x}$ (as quasistable curves) outside of $0 \in \mathbb{P}^{1}$ (whose images to $\mathscr{B}$ have their degrees sum up to $\left(\beta_{0}^{\prime}-\beta_{0}\right)$ ). (This does not change the defect of $x$ at 0 , and also does not change $\Theta(x)$.) Then we shrink all the inserting points of $C^{\prime}$ on $\mathbb{P}^{1}$ to 0 to obtain $\tilde{x}^{\prime} \in \mathscr{G}_{\mathscr{B}_{2, \beta}^{b}}$ in the limit, which exists by the valuative criterion of properness, as $\mathscr{G}_{2, \beta}^{b}$ is projective. By examining the images of this family on $\mathscr{Q}\left(\beta, w_{0}\right)$ via $\pi_{2, \beta}$, we deduce $y=\pi_{2, \beta}\left(\tilde{x}^{\prime}\right) \in \mathscr{Z}$. Therefore, the semicontinuity of $\Theta$ implies that $\Theta(x) \leq \Theta(y)=1-$ that is, $\Theta(x)=1$. Hence, $\mathscr{B}_{2, \beta_{0}}\left[w, w_{0}\right]$ must be connected. This is a contradiction, and we conclude that $\Theta \equiv 1$ (for general $(v, w) \in\left\{\left(e, w_{0}\right),\left(w_{0}, w_{0}\right)\right\}$ by the previous two paragraphs).

Therefore, Proposition 5.13 implies the result.

Corollary 5.17. Set $\beta \in Q_{+}^{\vee}$ and $w \in W$. For each $i \in \mathrm{I}$ such that $s_{i} w<w$, we have a surjective map

$$
\pi_{i}: P_{i} \times{ }^{B} Q(\beta, w) \rightarrow Q Q\left(\beta, s_{i} w\right)
$$

such that $\left(\pi_{i}\right)_{*} \mathcal{O}_{P_{i} \times^{B} Q(\beta, w)} \cong \mathcal{O}_{Q}\left(\beta, s_{i} w\right)$ and $\mathbb{R}^{>0}\left(\pi_{i}\right)_{*} \mathcal{O}_{P_{i} \times^{B} Q(\beta, w)} \cong\{0\}$.

Proof. Combine Corollary 4.40 with Theorem 4.30 (and take the generic localisations over $\mathbb{Z}$ ).

Corollary 5.18. For each $\beta \in Q_{+}^{\vee}$ and $w \in W$, the variety $Q(\beta, w)$ is normal.

Proof. The case $w=w_{0}$ is in Theorem 5.16. Assume that the assertion holds for $w$. Set $i \in \mathrm{I}$ such that $s_{i} w<w$. Then Corollary 5.17 implies that $\mathcal{O}_{\mathscr{Q}\left(\beta, s_{i} w\right)}$ is isomorphic to the normal sheaf of rings $\left(\pi_{i}\right)_{*} \mathcal{O}_{P_{i} \times B} Q(\beta, w)$. Hence the assertion holds for $s_{i} w<w$. This proceeds to the induction, and we conclude the result.

Corollary 5.19. For each $\beta \in Q_{+}^{\vee}$ and $w, v \in W$, the subspace

$$
\mathrm{e}_{1}^{-1}\left(p_{v}\right) \cap \mathrm{e}_{2}^{-1}(\mathscr{B}(w)) \subset \mathscr{B}_{2, \beta}
$$

is connected.

Proof. This space appears in the fibre of $\pi_{\beta, w_{0}, w}$ along the constant quasi-map $\mathbb{P}^{1} \rightarrow\left\{p_{v}\right\} \subset \mathscr{B}$ with its defect concentrated in $0 \in \mathbb{P}^{1}$. Hence, the assertion follows from Corollary 5.18 and Proposition 5.13 .

Theorem 5.20. For each $\beta \in Q_{+}^{\vee}$ and $w, v \in W$, the scheme $Q(\beta, v, w)$ is normal.

Proof. The combination of Theorem 5.7 and Corollary 5.19 implies that every fibre of $\pi_{\beta, v, w}$ is connected. Thus, Proposition 5.13 implies the result. 
Corollary 5.21. For each $\mathrm{J} \subset \mathrm{I}, \beta \in Q_{+}^{\vee}$ and $w, v \in W$, the variety $Q_{\mathrm{J}}(\beta, v, w)$ is irreducible and normal.

Proof. By Corollary 4.35 and Remark 4.36, we can rearrange the map $\Pi_{\mathrm{J}}$ to be surjective with connected fibres. Hence, Proposition 5.3 implies the irreducibility of $Q_{\mathrm{J}}(\beta, v, w)$. By the Stein factorisation theorem applied to the composition map $\Pi_{\mathrm{J}} \circ \pi_{\beta, v, w}$, we deduce that the normalisation of $Q_{\mathrm{J}}(\beta, v, w)$ is bijective to $Q_{\mathrm{J}}(\beta, v, w)$. Therefore, the weak normality of $\mathscr{Q}_{\mathrm{J}}(\beta, v, w)$ (Corollary 4.42) implies the result.

We set $W^{\mathrm{J}}:=W / w_{0} W_{\mathrm{J}} w_{0}$ and identify it with the set of their minimal length representatives in $W$. Recall that $2 \rho_{\mathrm{J}}$ is the sum of the positive roots that belong to the unipotent radical of $\mathfrak{p}(\mathrm{J})$.

Corollary 5.22. For each $\mathrm{J} \subset \mathrm{I}, \beta \in Q_{+}^{\vee}$ such that $-w_{0} \beta \in Q_{\mathrm{j},+}^{\vee}, w \in W^{\mathrm{J}}$ and $v \in W$ such that $w_{0} v \in W^{\mathrm{J}}$, the variety $Q_{\mathrm{J}}(\beta, v, w)$ has dimension

$$
\operatorname{dim} G / P(\mathrm{~J})-2\left\langle w_{0} \beta, \rho_{\mathrm{J}}\right\rangle+\ell(v)-\ell\left(w_{0}\right)-\ell(w)
$$

if it is nonempty. When $v=w_{0}$, the variety $\mathbb{Q}_{\mathrm{J}}(\beta, v, w)$ is always nonempty. In addition, $\mathbb{Q}_{\mathrm{J}}\left(\beta, w_{0}, e\right)$ is the collection of DP-data as in Definition 2.6, with the data and compatibility conditions only for $\lambda \in P_{\mathrm{J},+}$.

Remark 5.23. If we replace the labels of $Q_{\jmath}(\beta, v, w)=Q_{\jmath}^{\prime}\left(v t_{\beta}, w\right)_{\mathbb{C}}$ with the labels of parabolic semiinfinite Bruhat order as in $[61, \S 10.3]$, then we can write the condition of $Q_{J}(\beta, v, w)$ to be nonempty by the order relation, just as in Corollary 4.21.

Proof of Corollary 5.22. By [12], the collection of DP-data in Definition 2.6 with the compatibility condition imposed only for $\lambda \in P_{\mathrm{J},+}$ (without nondefining points) represents the (closure of the) space of maps $\mathbb{P}^{1} \rightarrow G / P(\mathrm{~J})$ such that the image of $\left[\mathbb{P}^{1}\right]$ in $\mathscr{B}$ is $-w_{0} \beta \in H_{2}(\mathscr{B}, \mathbb{Z})$. Thus, we conclude the last assertion.

The dimension of $Q_{\mathrm{J}}\left(\beta, w_{0}, e\right)$ is given by $\operatorname{dim} G / P(\mathrm{~J})-2\left\langle w_{0} \beta, \rho_{\mathrm{J}}\right\rangle$, since the first Chern class of the tangent bundle of $G / P(\mathrm{~J})$ is $2 \rho_{\mathrm{J}}$ (compare [29, Proposition 3.5]). In particular, the dimension of $Q_{\mathrm{J}}\left(\beta, w_{0}, e\right)$ is formula $(5.4)$ in this case.

Since imposing a hyperplane section in the image of $G / P(\mathrm{~J})$ through the (generically defined) evaluation map at $0 \in \mathbb{P}^{1}$ (which is surjective as being $G$-equivariant)

$$
Q_{\mathrm{J}}\left(\beta, w_{0}, e\right) \rightarrow G / P(\mathrm{~J})
$$

lowers the dimension by exactly 1 , we deduce that $\mathbb{Q}_{J}\left(\beta, w_{0}, w\right)$ has an irreducible component of expected dimension. From this and Corollary 5.21, we conclude that $Q_{\mathrm{J}}\left(\beta, w_{0}, w\right)$ is nonempty and its dimension is given by formula (5.4).

We consider the dimension estimate in the general case.

Since $\mathbb{Q}_{\mathrm{J}}\left(\beta, s_{i} v, w\right) \subsetneq \mathbb{Q}_{\mathrm{J}}(\beta, v, w)(i \in \mathrm{I})$ implies $s_{i} v>\frac{\infty}{2} v\left(\Leftrightarrow s_{i} v<v\right)$ when $w_{0} s_{i} v \in W^{\mathrm{J}}$, we find that they are the images of $\mathbb{Q}\left(\beta^{\prime}, s_{i} v, w\right) \subsetneq \mathscr{Q}\left(\beta^{\prime}, v, w\right)$ for some $\beta^{\prime} \in \beta+\sum_{j \in \mathrm{J}} \mathbb{Z}_{\geq 0} \alpha_{j}^{\vee}$ (see Remark 4.36). In particular, $Q_{\mathrm{J}}(\beta, v, w)$ has dimension of at least formula (5.4) in general, by induction from the case when $v=w_{0}$.

Let $Z$ denote the space of genus 0 stable maps to $\mathbb{P}^{1} \times G / P(\mathrm{~J})$ of bidegree $(1, \beta)$ with two marked points such that the first and second marked points correspond to $0, \infty \in \mathbb{P}^{1}$, respectively (parabolic analogue of $\left.\mathscr{G}_{2, \beta}^{b}\right)$. Then we have a resolution $\pi: Z \rightarrow Q_{\mathrm{J}}\left(\beta, w_{0}, e\right)$ (which shares genuine maps $\mathbb{P}^{1} \rightarrow G / P(\mathrm{~J})$ in their common open dense subset). Let $\eta: \mathscr{B}=G / B \rightarrow G / P(\mathrm{~J})$ be the projection. By our choices of $v, w$, we find

$$
\operatorname{codim}_{\mathscr{B}} \mathscr{B}(w)=\operatorname{codim}_{G / P(\mathrm{~J})} \eta(\mathscr{B}(w)) \quad \text { and } \operatorname{codim}_{\mathscr{B}} \mathscr{B}^{\mathrm{op}}(v)=\operatorname{codim}_{G / P(\mathrm{~J})} \eta\left(\mathscr{B}^{\mathrm{op}}(v)\right),
$$


respectively. We have the evaluation map of two points $\mathrm{z}: Z \rightarrow(G / P(\mathrm{~J}))^{2}$. We set $Z_{v, w}:=$ $\mathrm{z}^{-1}\left(\eta(\mathscr{B}(w)) \times \eta\left(\mathscr{B}^{\mathrm{op}}(v)\right)\right)$. By [16, Propostion $\left.\left.3.2 \mathrm{~b}\right)\right]$ (see also the proof of Corollary 5.2), we find that

$$
\begin{aligned}
\operatorname{dim} Z_{v, w} & =\operatorname{dim} Z-\operatorname{codim}_{G / P(\mathrm{~J})} \eta(\mathscr{B}(w))-\operatorname{codim}_{G / P(\mathrm{~J})} \eta\left(\mathscr{B}^{\mathrm{op}}(v)\right) \\
& =\operatorname{dim} Z-\operatorname{codim}_{\mathscr{B}} \mathscr{B}(w)-\operatorname{codim}_{\mathscr{B}} \mathscr{B}^{\mathrm{op}}(v)=(5.4),
\end{aligned}
$$

and $Z_{v, w}$ is irreducible (if it is nonempty). Since $G / P(\mathrm{~J})$ is homogeneous, we find that $G \cdot \pi\left(Z_{w_{0}, w}\right) \subset Z$ is Zariski dense. Hence, $Z_{w_{0}, w}$ contains an open dense subset whose points are genuine maps $\mathbb{P}^{1} \rightarrow$ $G / P(J)$. In conjunction with the irreducibility of $Z_{w_{0}, w}$ and $Q_{\jmath}\left(\beta, w_{0}, w\right)$, we find $\pi^{-1}\left(Q_{\jmath}\left(\beta, w_{0}, w\right)\right)=$ $Z_{w_{0}, w}$. Similarly, we have $\pi^{-1}\left(Q_{\jmath}(\beta, v, e)\right)=Z_{v, e}$. Therefore, we conclude that $Z_{v, w}$ surjects onto $\mathbb{Q}_{\mathrm{J}}(\beta, v, w)$ by the restriction of $\pi$. In particular, $Q_{\mathrm{J}}(\beta, v, w)$ has dimension of at most formula (5.4) in general.

Therefore, our dimension estimate must be strict in general.

Corollary 5.24. For each $\mathrm{J} \subset \mathrm{I}$ and $w, v \in W_{\mathrm{a}}$, the scheme ${Q_{\mathrm{J}}^{\prime}}(v, w)_{\overline{\mathbb{F}}_{p}}$ is irreducible and normal for $p \gg 0$.

Proof. Apply Proposition 4.43 to Corollary 5.21 (compare Lemma 4.6).

Corollary 5.25. Let $\mathbb{K}$ be an algebraically closed field of characteristic 0 or $p \gg 0$. For each $\mathrm{J} \subset \mathrm{I}$ and $w, v \in W_{\mathrm{af}}$, the scheme $\mathcal{Q}_{\mathrm{J}}^{\prime}(v, w)_{\mathbb{K}}$ is projectively normal with respect to a line bundle $\mathcal{O}_{\mathbb{Q}_{\mathrm{J}}^{\prime}(v, w)_{\mathbb{K}}}(\lambda)$ $\left(\lambda \in P_{\mathrm{J},++}\right)$.

Proof. In view of Lemmas 4.1 and 4.3 and Theorem 4.33, the multiplication of the section ring afforded by $\left\{\mathcal{O}_{Q_{\jmath}^{\prime}(v, w)_{\mathbb{K}}}(m \lambda)\right\}_{m \geq 0}$ is surjective. Therefore, Corollaries 5.21 and 5.24 imply the result.

\section{A. Some properties of $\mathbf{Q}_{G}(w)$ in positive characteristic}

We work in the setting of Section 4.2. Let $\mathbb{K}$ be an algebraically closed field of characteristic $\neq 2$. The aim of this appendix is twofold: to show that our scheme $\mathbf{Q}_{G, \mathrm{~J}}(w)_{\mathbb{R}}$ is (projectively) normal and to present an analogue of the Kempf vanishing theorem for $\mathbf{Q}_{G}(w)_{\mathbb{K}}$. Both results are proved in [51] for the case when $\mathbb{K}=\mathbb{C}$. Our proof of the former is new and does not depend on [51] (when $w=e$ ), while our proof of the latter depends on the results and arguments in [51] in an essential way.

Proposition A.1. For each $w \in W_{\mathrm{af}}$ and $\mathrm{J} \subset \mathrm{I}$, the ring $R_{w}(\mathrm{~J})_{\mathbb{K}}$ is normal.

Proof. We first prove the case where $w=e$ and J $=\emptyset$. Let $\mathbf{Q}_{G}$ denote the open $G[[z]]$-orbit of $\mathbf{Q}_{G}(e)_{\mathbb{K}}$ obtained by the $G$-translation of Lemma 4.11.

We have an inclusion

$$
\mathbb{W}(\lambda)_{\mathbb{K}}^{\vee} \subset \Gamma\left(\grave{\mathbf{Q}}_{G}, \mathcal{O}_{\mathbf{Q}_{G}(e)_{\mathbb{K}}}(\lambda)\right),
$$

since $R_{\mathbb{K}}$ is integral (Corollary 4.12). We also have an inclusion

$$
\Gamma\left(\stackrel{\mathbf{Q}}{G}_{G}, \mathcal{O}_{\mathbf{Q}_{G}(e)_{\mathbb{R}}}(\lambda)\right) \hookrightarrow \Gamma\left(\mathbb{O}(e)_{\mathbb{R}}, \mathcal{O}_{\mathbf{Q}_{G}}(e)_{\mathbb{K}}(\lambda)\right), \quad \lambda \in P_{+} .
$$

Thanks to Lemma 4.11 and its proof, we deduce

$$
\Gamma\left(\mathbb{O}(e)_{\mathbb{K}}, \mathcal{O}_{\mathbf{Q}_{G}(e)_{\mathbb{K}}}(\lambda)\right) \cong \mathbb{K}[\mathbf{I} /(\mathbf{I} \cap H N((z)))] \otimes_{\mathbb{K}} \mathbb{K}_{-w_{0} \lambda},
$$

which is cocyclic as a $U_{\mathbb{K}}^{+}$-module. Since the $G$-action on $\Gamma\left(\grave{\mathbf{Q}}_{G}, \mathcal{O}_{\mathbf{Q}_{G}(e)_{\mathbb{K}}}(\lambda)\right)$ is algebraic, we deduce that

$$
\left(\mathbb{K}[\mathbf{I} /(\mathbf{I} \cap H N((z)))] \otimes_{\mathbb{K}} \mathbb{K}_{-w_{0} \lambda}\right)^{\vee} \longrightarrow \Gamma\left(\stackrel{\mathbf{Q}}{G}_{G}, \mathcal{O}_{\mathbf{Q}_{G}}(e)_{\mathbb{K}}(\lambda)\right)^{\vee}
$$


is a $\dot{U}_{\mathbb{K}}^{0}$-integrable quotient. By Proposition 3.19, we conclude a surjection

$$
\mathbb{W}(\lambda)_{\mathbb{K}} \longrightarrow \Gamma\left(\stackrel{\mathbf{Q}}{G}_{G}, \mathcal{O}_{\mathbf{Q}_{G}(e)_{\mathbb{K}}}(\lambda)\right)^{\vee}
$$

Compared with formula (A.1), we conclude the isomorphism

$$
\Gamma\left(\stackrel{\mathbf{Q}}{G}_{G}, \mathcal{O}_{\mathbf{Q}_{G}}(e)_{\mathbb{R}}(\lambda)\right) \stackrel{\cong}{\longrightarrow} \Gamma\left(\mathbf{Q}_{G}(e)_{\mathbb{K}}, \mathcal{O}_{\mathbf{Q}_{G}(e)_{\mathbb{K}}}(\lambda)\right), \quad \lambda \in P_{+},
$$

since the space of sections supported on a dense open Zarsiki subset must be larger than (or equal to) the space of sections supported on the whole space for an integral scheme. In other words, $R_{\mathbb{K}}$ is the maximal $\dot{U}_{\mathbb{K}}^{0}$-integrable $U_{\mathbb{K}^{+}}^{+}$-stable subring of

$$
S_{\mathbb{K}}:=\bigoplus_{\lambda \in P_{+}} \Gamma\left(\mathbb{O}(e)_{\mathbb{K}}, \mathcal{O}_{\mathbf{Q}_{G}(e)_{\mathbb{K}}}(\lambda)\right)=\bigoplus_{\lambda \in P_{+}} \mathbb{K}[\mathbf{I} /(\mathbf{I} \cap H N((z)))] \otimes_{\mathbb{K}} \mathbb{K}_{-w_{0} \lambda}
$$

The ring $S_{\mathbb{K}}$ is integrally closed, as it is a polynomial ring (of countably many variables). Hence, the integral closure $R^{+}$of the ring $R_{\mathbb{K}}$ is also a subring of $S_{\mathbb{K}}$. In view of the $\dot{U}_{\mathbb{K}}^{0}$-integrability, the ring $R_{\mathbb{K}}$ admits an algebraic $G$-action. By the canonical nature of the normalisation, we deduce that $R^{+}$admits an algebraic $G$-action (note that we can approximate $R_{\mathbb{K}}$ as a union of Noetherian rings with algebraic $G$-actions). However, $R_{\mathbb{K}}$ is already the maximal $\dot{U}_{\mathbb{K}}^{0}$-integrable $U_{\mathbb{K}}^{+}$-stable subring of $S_{\mathbb{K}}$. Hence, we necessarily have $R^{+}=R_{\mathbb{K}}$. Thus, the case of $w=e$ and $\mathrm{J}=\emptyset$ follows.

The case of arbitrary $w \in W_{\text {af }}$ follows from the case of $w=e$ as in [46, §4]. As the fraction field of $R_{w}(\mathrm{~J})_{\mathbb{K}}$ is a subfield of the fraction field of $\left(R_{w}\right)_{\mathbb{K}}$ and $P_{\mathrm{J},+} \subset P_{+}$forms (the set of integral points of) a face, we conclude the general case by restriction.

Corollary A.2. The scheme $\mathbf{Q}_{G, \mathrm{~J}}(w)_{\mathbb{K}}$ is normal.

Corollary A.3. For each $\lambda \in P_{\mathrm{J},+}$, we have

$$
H^{0}\left(\mathbf{Q}_{G, j}(w)_{\mathbb{K}}, \mathcal{O}_{\mathbf{Q}_{G, J}(w)_{\mathbb{K}}}(\lambda)\right)=\mathbb{W}_{w w_{0}}(\lambda)_{\mathbb{K}}^{\vee}
$$

Proof. Because finding $H^{0}$ from $R_{w}(\mathrm{~J})_{\mathbb{K}}$ can be seen as finding graded pieces of the normalisation, the assertion follows from Proposition A.1.

Proposition A.4 ([51]). Assume that char $\mathbb{K} \neq 2$. Set $w \in W$ and $] \subset$ I. Then an I-equivariant line bundle on $\mathbf{Q}_{G, \mathrm{~J}}(w)_{\mathbb{K}}$ is a character twist of $\left\{\mathcal{O}_{\mathbf{Q}_{G, \mathrm{~J}}(w)_{\mathbb{K}}}(\lambda)\right\}_{\lambda \in P_{\mathrm{J}}}$.

Proof. Taking Corollaries 4.20 and A.2 into account, the proof in [51, Proposition 5.1] works in this setting.

Theorem A.5. Set $w \in W$ and $\mathrm{J} \subset \mathrm{I}$. For each $\lambda \in P_{\mathrm{J}}$, we have

$$
H^{>0}\left(\mathbf{Q}_{G, J}(w)_{\mathbb{K}}, \mathcal{O}_{\mathbf{Q}_{G, J}(w)_{\mathbb{K}}}(\lambda)\right)=\{0\} .
$$

Proof. Assume that $w=e$. We consider the subring defined by

$$
T_{\mathrm{J}}:=\bigoplus_{\lambda \in P_{\mathrm{J},+}} T_{\mathrm{J}}(\lambda) \subset \bigoplus_{\lambda \in P_{\mathrm{J},+}} \mathbb{W}(\lambda)_{\mathbb{K}}^{\vee}=\bigoplus_{\lambda \in P_{\mathrm{J},+}} \mathbb{W}_{w_{0}}(\lambda)_{\mathbb{K}}^{\vee}=R_{\mathrm{J}},
$$

where $T_{\mathrm{J}}(\lambda) \subset \mathbb{W}(\lambda)_{\mathbb{K}}^{\vee}$ is the $P$-weight $(-\lambda)$-part of $\mathbb{W}(\lambda)_{\mathbb{K}}^{\vee}$. They are generated by the duals of $\widetilde{P}_{i, m \delta} \mathbf{v}_{\varpi_{i}} \in \mathbb{W}\left(\varpi_{i}\right)_{\mathbb{K}}\left(i \in \mathrm{I} \backslash \mathrm{J}, m \in \mathbb{Z}_{>0}\right.$; see [5, (3.18)] for the definition of $\left.\widetilde{P}_{i, m \delta}\right)$. In view of [5, Proposition 3.18], the ring $T_{\mathrm{J}}$ is a polynomial ring. The actions of $\left\{\widetilde{P}_{i, m \delta}\right\}_{i \in(\mathrm{I} \backslash \mathrm{J}), m>0}$ define endomorphisms of the module $\mathbb{W}(\lambda)_{\mathbb{K}}\left(\lambda \in P_{\mathrm{J},+}\right)$, each of which is either an injection or zero (compare the proof of Proposition 3.19). It follows that the action of $T_{\mathrm{J}}$ on $R_{\mathrm{J}}$ is torsion free. 
Thanks to [51, Corollary 4.29], the character comparison forces the torsion-free action of $T_{\mathrm{J}}$ on $R_{\mathrm{J}}$ to be free. Therefore, the proof of [51, Theorem 4.30] works in a verbatim way. This proves the case when $w=e$. The general case can be easily deduced from the $w=e$ case as in [51, Corollary 4.31].

\section{B. An application of the Pieri-Chevalley formula}

We work in the setting of Section 4.4. The aim of this appendix is to present a method (Theorem B.6) to describe the global sections of nef line bundles on $Q^{\prime}(v, w)_{\mathbb{R}}$ for $v, w \in W_{\text {af }}$ and an algebraically closed field $\mathbb{K}$ of characteristic $\neq 2$.

For each $\mu \in P_{+}$, we have an extremal weight module $\mathbb{X}(\mu)_{\mathbb{K}}$ and its global base $\mathbf{B}(\mathbb{X}(\mu))$ borrowed from Theorem 3.14.

Lemma B.1. Set $\mathrm{J} \subset \mathrm{I}$ and $\mu \in P_{\mathrm{J}^{+}+}$. A subset of $\mathbf{B}(\mathbb{X}(\mu))$ spans

$$
H^{0}\left(\mathbf{Q}_{G}(w)_{\mathbb{K}}, \mathcal{O}_{\mathbf{Q}_{G}(w)_{\mathbb{K}}}(\mu)\right)^{\vee}=H^{0}\left(\mathbf{Q}_{G, \mathrm{~J}}(w)_{\mathbb{K}}, \mathcal{O}_{\mathbf{Q}_{G, \mathrm{~J}}(w)_{\mathbb{K}}}(\mu)\right)^{\vee} \subset \mathbb{X}(\mu)_{\mathbb{K}}
$$

and

$$
\begin{aligned}
& \theta\left(H^{0}\left(\mathbf{Q}_{G}\left(v w_{0}\right)_{\mathbb{K}}, \mathcal{O}_{\mathbf{Q}_{G}\left(v w_{0}\right)_{\mathbb{K}}}\left(-w_{0} \mu\right)\right)^{\vee}\right)
\end{aligned}
$$

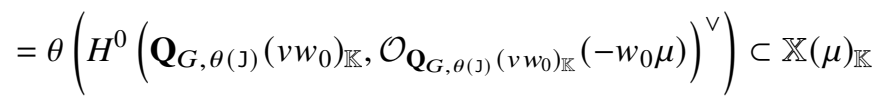

for each $w, v \in W_{\mathrm{af}}$.

Proof. Taking Corollary A.3 into account, the assertion follows from Theorem 3.14.

Corollary A. 3 combined with Theorem 4.33 yields

$$
H^{0}\left({Q^{\prime}}^{\prime}(v, w)_{\mathbb{K}}, \mathcal{O}_{Q^{\prime}(v, w)_{\mathbb{K}}}(\mu)\right)^{\vee} \hookrightarrow H^{0}\left(\mathbf{Q}_{G}(w)_{\mathbb{K}}, \mathcal{O}_{\mathbf{Q}_{G}(w)_{\mathbb{K}}}(\mu)\right)^{\vee} \subset \mathbb{X}(\mu)_{\mathbb{K}}
$$

for each $w, v \in W_{\text {af }}$.

Lemma B.2. Set $\mu \in P_{++}$. For each $b \in \mathbf{B}(\mathbb{X}(\mu))$, there exist unique elements $\kappa(b), \iota(b) \in W_{\text {af }}$ with the following properties:

1. $b \in H^{0}\left(\mathbf{Q}_{G}(w)_{\mathbb{K}}, \mathcal{O}_{\mathbf{Q}_{G}(w)_{\mathbb{K}}}(\mu)\right)^{\vee}$ for $w \in W_{\text {af }}$ if and only if $\kappa(b) \leq_{\frac{\infty}{2}} w$.

2. $b \in \theta\left(H^{0}\left(\mathbf{Q}_{G}\left(v w_{0}\right)_{\mathbb{K}}, \mathcal{O}_{\mathbf{Q}_{G}\left(v w_{0}\right)_{\mathbb{K}}}\left(-w_{0} \mu\right)\right)^{\vee}\right)$ for $v \in W_{\mathrm{af}}$ if and only if $\iota(b) \geq_{\frac{\infty}{2}} v$.

Proof. In view of Corollary A.3, the first assertion is a rephrasing of Lemma 3.15. The second assertion is obtained from the first in view of Lemma 3.17 and formula (2.2).

Corollary B.3. The functions $\kappa$ and $\mathrm{l}$ play the same role as the same-named functions in [51, (2.17)] (with the opposite convention on the order $\leq \frac{\infty}{2}$ ).

Proof. Compare Lemma B.2 with [51, Theorem 2.8].

Corollary B.4. Set $\mu \in P_{++}$and $v, w \in W_{\mathrm{af}}$. The space $H^{0}\left(\widehat{Q}^{\prime}(v, w)_{\mathbb{K}}, \mathcal{O}_{\mathbb{Q}^{\prime}(v, w)_{\mathbb{K}}}(\mu)\right)^{\vee}$ is spanned by the subset of $b \in \mathbf{B}(\mathbb{X}(\mu))$ that satisfies $w \geq_{\frac{\infty}{2}} \kappa(b) \geq_{\frac{\infty}{2}} l(b) \geq_{\frac{\infty}{2}} v$.

Proof. This is a rephrasing of Theorem 4.33 for $\mathrm{J}=\emptyset$.

Theorem B.5 ([51]). Set $w \in W_{\mathrm{af}}$ and $\lambda \in P_{+}$. There exists a unique collection of elements $a_{w}^{u}(\lambda) \in$ $\mathbb{Z}\left[q^{-1}\right][H]\left(u \in W_{\mathrm{af}}\right)$ such that

$$
\left.a_{w}^{w}(\lambda)=e^{-w w_{0} \lambda}, \quad a_{w}^{u}(\lambda)=0 \text { if } u \not\right\rfloor_{\frac{\infty}{2}} w
$$


and

$$
\operatorname{gch} \Gamma\left(\mathbf{Q}_{G}(w)_{\mathbb{K}}, \mathcal{O}_{\mathbf{Q}_{G}(w)_{\mathbb{K}}}(\lambda+\mu)\right)=\sum_{u \in W_{\mathrm{af}}} a_{w}^{u}(\lambda) \operatorname{gch} \Gamma\left(\mathbf{Q}_{G}(u)_{\mathbb{K}}, \mathcal{O}_{\mathbf{Q}_{G}(u)_{\mathbb{K}}}(\mu)\right)
$$

for every $\mu \in P_{++}$.

The goal of this appendix is to prove the following:

Theorem B.6. Set $w, v \in W_{\mathrm{af}}$ and $\lambda \in P_{+}$. Let $\left\{a_{w}^{v}(\lambda)\right\}_{v \in W_{\mathrm{af}}}$ be the collection of elements in Theorem B.5. Then we have

$$
\operatorname{gch} \Gamma\left(Q^{\prime}(v, w)_{\mathbb{K}}, \mathcal{O}_{\mathbb{Q}^{\prime}(v, w)_{\mathbb{K}}}(\lambda)\right)=\sum_{u \geq \frac{\infty}{2} v} a_{w}^{u}(\lambda) .
$$

Proof. The proof of the numerical part of Theorem B.5 is [51, Theorem 3.5] (proved in [51, §8.1]), and it counts the elements of $\mathbf{B}(\mathbb{X}(\lambda+\mu))$ that contribute $\Gamma\left(\mathbf{Q}_{G}(w)_{\mathbb{K}}, \mathcal{O}_{\mathbf{Q}_{G}(w)_{\mathbb{K}}}(\lambda+\mu)\right)^{\vee}=\mathbb{W}_{w w_{0}}(\lambda+\mu)_{\mathbb{K}}$ in two ways. In particular, we can additionally impose the condition $l(\bullet) \geq_{\frac{\infty}{2}} v$ for $\mathbf{B}(\mathbb{X}(\lambda+\mu))$ to deduce that

$$
\operatorname{gch} \Gamma\left(\mathcal{Q}^{\prime}(v, w)_{\mathbb{K}}, \mathcal{O}_{\mathbb{Q}^{\prime}(v, w)_{\mathbb{K}}}(\lambda+\mu)\right)=\sum_{u \leq \frac{\infty}{2} v} a_{w}^{u}(\lambda) \operatorname{gch} \Gamma\left(Q^{\prime}(u, w)_{\mathbb{K}}, \mathcal{O}_{\mathscr{Q}^{\prime}}(u, w)_{\mathbb{K}}(\mu)\right)
$$

for every $\mu \in P_{++}$in view of Corollary B.4. The $\left(H \times \mathbb{G}_{m}\right)$-equivariant Euler characteristic of $\mathcal{O}_{Q^{\prime}(v, w)_{\mathbb{K}}}(\mu)(\mu \in P)$ is a rational function on the characters of $H$, and we can specialise to $\mu=0$. Now we apply Theorem 4.33 to deduce

$$
\chi\left(Q^{\prime}(v, w)_{\mathbb{K}}, \mathcal{O}_{Q^{\prime}(v, w)_{\mathbb{K}}}(\mu)\right)=\operatorname{gch} \Gamma\left(Q^{\prime}(v, w)_{\mathbb{K}}, \mathcal{O}_{Q^{\prime}(v, w)_{\mathbb{K}}}(\mu)\right)
$$

for every $\mu \in P_{+}$. This implies the desired equality.

\section{Remark B.7.}

1. In view of Corollary 4.35 and Remark 4.36, Theorem B.6 describes the space of global sections of $\mathcal{O}_{Q_{j}^{\prime}(v, w)_{\mathbb{K}}}(\lambda)$ for every $w, v \in W_{\mathrm{af}}, \mathrm{J} \subset \mathrm{I}$ and $\lambda \in P_{\mathrm{J},+}$.

2. In conjunction with Theorem 4.33, Theorem B.6 can be seen as an analogue of [59, Theorem 34] for semi-infinite flag manifolds.

3. Thanks to [51, §3], we have a combinatorial rule to express $a_{w}^{u}(\lambda) \mathrm{s}$.

Acknowledgments. The author would like to thank Prakash Belkale, Ievgen Makedonskyi, Leonardo Mihalcea, Katsuyuki Naoi and Daisuke Sagaki for helpful discussions. This work was supported in part by JSPS KAKENHI grants JP26287004 and JP19H01782.

Conflict of Interest: None.

\section{References}

[1] H. H. Andersen, P. Polo and K. Wen, 'Representations of quantum algebras', Invent. Math. 104 (1991), 1-59.

[2] S. Arkhipov, R. Bezrukavnikov, A. Braverman, D. Gaitsgory and I. Mirković, 'Modules over the small quantum group and semi-infinite flag manifold', Transform. Groups 10(3-4) (2005), 279-362.

[3] A. Beauville and Y. Laszlo, 'Conformal blocks and generalized theta functions', Comm. Math. Phys. 164(2) (1994), 385-419.

[4] J. Beck, V. Chari and A. Pressley, 'An algebraic characterization of the affine canonical basis', Duke Math. J. 99(3) (1999), $455-487$.

[5] J. Beck and H. Nakajima, 'Crystal bases and two-sided cells of quantum affine algebras', Duke Math. J. 123(2) (2004), $335-402$.

[6] K. A. Behrend and Y. I. Manin, 'Stacks of stable maps and Gromov-Witten invariants', Duke Math. J. 85(1) (1996), 1-60.

[7] A. Braverman, 'Spaces of quasi-maps into the flag varieties and their applications', in International Congress of Mathematicians, Vol. II (European Mathematical Society, Zürich, 2006), 1145-1170. 
[8] A. Braverman, B. Feigin, M. Finkelberg and L. Rybnikov, 'A finite analog of the AGT relation I: Finite $W$-algebras and quasimaps' spaces', Comm. Math. Phys. 308(2) (2011), : 457-478.

[9] A. Braverman and M. Finkelberg, 'Semi-infinite Schubert varieties and quantum $K$-theory of flag manifolds', J. Amer. Math. Soc. 27(4) (2014), 1147-1168.

[10] A. Braverman and M. Finkelberg, 'Weyl modules and $q$-Whittaker functions', Math. Ann. 359(1-2) (2014), 45-59.

[11] A. Braverman and M. Finkelberg, 'Twisted zastava and $q$-Whittaker functions', J. London Math. Soc. (2) 96(2) (2017), 309-325.

[12] A. Braverman, M. Finkelberg, D. Gaitsgory and I. Mirković, 'Intersection cohomology of Drinfeld's compactifications', Selecta Math. (N.S.) 8(3) (2002), 381-418.

[13] A. Braverman and D. Gaitsgory, 'Geometric Eisenstein series', Invent. Math. 150(2) (2002), 287-384.

[14] M. Brion and S. Kumar, Frobenius Splitting Methods in Geometry and Representation Theory, Progress in Mathematics vol. 231 (Birkhäuser Boston, Inc., Boston, MA, 2005).

[15] M. Brion and V. Lakshmibai, 'A geometric approach to standard monomial theory', Represent. Theory 7 (2003), 651-680.

[16] A. S. Buch, P.-E. Chaput, L. C. Mihalcea and N. Perrin, 'Finiteness of cominuscule quantum K-theory', Ann. Sci. Éc. Norm. Supér. (4) 46(3) (2013), 477-494.

[17] V. Chari, G. Fourier and T. Khandai, 'A categorical approach to Weyl modules', Transform. Groups 15 (2010), 517-549.

[18] V. Chari and B. Ion, 'BGG reciprocity for current algebras', Compos. Math. 151(7) (2015), 1265-1287.

[19] V. Chari and A. Pressley, 'Weyl modules for classical and quantum affine algebras', Represent. Theory 5 (2001), $191-223$.

[20] I. Cherednik and S. Kato, 'Nonsymmetric Rogers-Ramanujan sums and thick Demazure modules', Adv. Math. 374 (2020), 107335.

[21] N. Chriss and V. Ginzburg, Representation Theory and Complex Geometry, Modern Birkhäuser Classics (Birkhäuser Boston, Inc., Boston, MA, 2010). Reprint of the 1997 edition.

[22] G. Faltings, 'Algebraic loop groups and moduli spaces of bundles', J. Eur. Math. Soc. (JEMS) 5(1) (2003), 41-68.

[23] B. Feigin, M. Finkelberg, A. Kuznetsov and I. Mirković, 'Semi-infinite flags. II. Local and global intersection cohomology of quasimaps' spaces', in Differential Topology, Infinite-Dimensional Lie Algebras, and Applications, American Mathematical Society Translations Series 2 vol. 194 of Amer. Math. Soc. Transl. Ser. 2 (American Mathematical Society, Providence, RI, 1999), 113-148.

[24] B. Feigin and E. Frenkel, 'Affine Kac-Moody algebras and semi-infinite flag manifold', Comm. Math. Phys. 128 (1990), 161-189.

[25] E. Feigin, S. Kato and I. Makedonskyi, 'Representation theoretic realization of non-symmetric Macdonald polynomials at infinity', J. Reine Angew. Math. 764 (2020), 181-216.

[26] E. Feigin and I. Makedonskyi, 'Vertex algebras and coordinate rings of semi-infinite flags', Comm. Math. Phys. 369(1) (2019), 221-244.

[27] E. Feigin and I. Makedonskyi, 'Semi-infinite Plücker relations and Weyl modules', Int. Math. Res. Not. IMRN 2020(14) (2020), 4357-4394.

[28] E. Feigin, I. Makedonskyi and D. Orr, 'Generalized Weyl modules and nonsymmetric $q$-Whittaker functions', Adv. Math. 339 (2018), 997-1033.

[29] M. Finkelberg and I. Mirković, 'Semi-infinite flags. I. Case of global curve $\mathbf{P}^{1}$ ', in Differential Topology, Infinite-Dimensional Lie Algebras, and Applications, American Mathematical Society Translations Series 2 vol. 194 (American Mathematical Society, Providence, RI, 1999), 81-112.

[30] W. Fulton and R. Pandharipande, 'Notes on stable maps and quantum cohomology', in Algebraic Geometry: Santa Cruz 1995 (American Mathematical Society, Providence, RI, 1995), Proc. Sympos. Pure Math., 62, Part 2, 45-96.

[31] H. Garland, 'The arithmetic theory of loop algebras', J. Algebra 53 (1978), 480-551.

[32] A. Givental, 'Homological geometry and mirror symmetry', in Proceedings of the International Congress of Mathematicians 1994 (Birkhäuser, Basel, 1995), 472-480.

[33] A. Givental and Y.-P. Lee, 'Quantum $K$-theory on flag manifolds, finite-difference Toda lattices and quantum groups', Invent. Math. 151(1) (2003), 193-219.

[34] A. B. Givental, 'Equivariant Gromov-Witten invariants', Int. Math. Res. Not. IMRN 13 (1996), 613-663.

[35] I. Grojnowski and G. Lusztig, 'A comparison of bases of quantized enveloping algebras', in Linear Algebraic Groups and Their Representations (Los Angeles, CA, 1992), Contemporary Mathematics vol. 153 (American Mathematical Society, Providence, RI, 1993), 11-19.

[36] A. Grothendieck, 'Éléments de géométrie algébrique. IV. Étude locale des schémas et des morphismes de schémas. III', Publ. Math. Inst. Hautes Études Sci. 28 (1966), 5-255.

[37] R. Hartshorne, Algebraic Geometry, Graduate Texts in Mathematics vol. 52 (Springer-Verlag, New York, 1977).

[38] N. Iwahori and H. Matsumoto, 'On some Bruhat decomposition and the structure of the Hecke rings of $p$-adic Chevalley groups', Publ. Math. Inst. Hautes Études Sci. 25 (1965), 5-48.

[39] A. Joseph, 'On the Demazure character formula', Ann. Sci. Éc. Norm. Supér. (4) 18(3) (1985), 389-419.

[40] V. G. Kac, Infinite-Dimensional Lie Algebras, third edn (Cambridge University Press, Cambridge, UK, 1990).

[41] M. Kashiwara, 'On crystal bases of the $q$-analogue of universal enveloping algebras', Duke Math. J. 63(2) (1991), $465-516$.

[42] M. Kashiwara, 'The crystal base and Littelmann's refined Demazure character formula', Duke Math. J. 71(3) (1993), 839858 . 
[43] M. Kashiwara, 'Crystal bases of modified quantized enveloping algebra', Duke Math. J. 73(2) (1994), $383-413$.

[44] M. Kashiwara, 'On level-zero representations of quantized affine algebras', Duke Math. J. 112(1) (2002), 117-175.

[45] M. Kashiwara, 'Level zero fundamental representations over quantized affine algebras and Demazure modules', Publ. Res. Inst. Math. Sci. 41(1) (2005), 223-250.

[46] S. Kato, 'Demazure character formula for semi-infinite flag varieties', Math. Ann. 371(3) (2018), 1769-1801.

[47] S. Kato, 'Loop structure on equivariant $K$-theory of semi-infinite flag manifolds', Preprint, 2018, arXiv:1805.01718v6.

[48] S. Kato, 'On quantum $K$-groups of partial flag manifolds', Preprint, 2019, arXiv:1906.09343.

[49] S. Kato, 'Frobenius splitting of thick flag manifolds of Kac-Moody algebras', Int. Math. Res. Not. IMRN 2020(17) (2020), 5401-5427.

[50] S. Kato and S. Loktev, 'A Weyl module stratification of integrable representations', Comm. Math. Phys. 368 (2019), $113-141$.

[51] S. Kato, S. Naito and D. Sagaki, 'Equivariant $K$-theory of semi-infinite flag manifolds and the Pieri-Chevalley formula', Duke Math. J. 169(13) (2020), 2421-2500.

[52] G. R. Kempf, 'Linear systems on homogeneous spaces', Ann. of Math. (2) 103(3) (1976), 557-591.

[53] B. Kim and R. Pandharipande, 'The connectedness of the moduli space of maps to homogeneous spaces', in Symplectic Geometry and Mirror Symmetry (Seoul, 2000) (World Scientific Publishing, River Edge, NJ, 2001), 187-201.

[54] S. L. Kleiman, 'The transversality of a general translate', Compos. Math. 28(3) (1974), 287-297.

[55] J. Kollár and S. Mori, Birational Geometry of Algebraic Varieties, Cambridge Tracts in Mathematics vol. 134 (Cambridge University Press, Cambridge, UK, 1998). With the collaboration of C. H. Clemens and A. Corti, translated from the 1998 Japanese original.

[56] M. Kontsevich and Y. Manin, 'Gromov-Witten classes, quantum cohomology, and enumerative geometry', Comm. Math. Phys. 164(3) (1994), 525-562.

[57] S. Kumar, Kac-Moody Groups, Their Flag Varieties and Representation Theory, Progress in Mathematics vol. 204 (Birkhäuser Boston, Inc., Boston, MA, 2002).

[58] S. Kumar and P. Littelmann, 'Algebraization of Frobenius splitting via quantum groups', Ann. of Math. (2) 155(2) (2002), 491-551.

[59] V. Lakshmibai and P. Littelmann, 'Richardson varieties and equivariant $K$-theory', J. Algebra 260(1) (2003), 230-260.

[60] T. Lam, C. Li, L. C. Mihalcea and M. Shimozono, 'A conjectural Peterson isomorphism in K-theory', J. Algebra 513 (2018), 326-343.

[61] T. Lam and M. Shimozono, 'Quantum cohomology of $G / P$ and homology of affine Grassmannian', Acta Math. 204(1) (2010), 49-90.

[62] C. Lenart, S. Naito, D. Sagaki, A. Schilling and M. Shimozono, 'A uniform model for Kirillov-Reshetikhin crystals II. Alcove model, path model, and $\mathrm{P}=\mathrm{X}$ ', Preprint, 2014, arXiv: 1402.2203.

[63] P. Littelmann, 'Contracting modules and standard monomial theory for symmetrizable Kac-Moody algebras', J. Amer. Math. Soc. 11(3) (1998), 551-567.

[64] G. Lusztig, 'Hecke algebras and Jantzen's generic decomposition patterns', Adv. Math. 37(2) (1980), $121-164$.

[65] G. Lusztig, 'Canonical bases in tensor products', Proc. Natl. Acad. Sci. USA 89(17) (1992), 8177-8179.

[66] G. Lusztig, Introduction to Quantum Groups, Progress in Mathematics vol. 110 (Birkhäuser, Boston, 1994).

[67] G. Lusztig, 'Study of a $\mathbf{z}$-form of the coordinate ring of a reductive group', J. Amer. Math. Soc. 22 (2009), 739-769.

[68] M. Manaresi, 'Some properties of weakly normal varieties', Nagoya Math. J. 77 (1980), 61-74.

[69] O. Mathieu, 'Formules de caractères pour les algèbres de Kac-Moody générales', Astérisque 159-160 (1988), 1-267.

[70] I. Mirković and K. Vilonen, 'Geometric Langlands duality and representations of algebraic groups over commutative rings', Ann. of Math. (2) 166(1) (2007), 95-143.

[71] M. Mustata, 'Jet schemes of locally complete intersection canonical singularities', 'Invent. Math. 424 (2001), $397-424$.

[72] S. Naito and D. Sagaki, 'Demazure submodules of level-zero extremal weight modules and specializations of Macdonald polynomials', Math. Z. 283 (2016), 937-978.

[73] K. Naoi, 'Tensor products of Kirillov-Reshetikhin modules and fusion products', Int. Math. Res.Not. IMRN 2017(18) (2017), 5667-5709.

[74] D. Peterson, 'Quantum cohomology of $G / P$ ', lecture at MIT (1997).

[75] A. Ramanathan, 'Equations defining Schubert varieties and Frobenius splitting of diagonals', Publ. Math. Inst. Hautes Études Sci. 65 (1987), 61-90.

[76] R. W. Richardson, 'Intersections of double cosets in algebraic groups', Indag.Math. (N.S.) 3 (1992), 69-77.

[77] The Stacks project authors, The Stacks Project (2018). URL: https://stacks.math.columbia.edu.

[78] E. Viehweg, Quasi-Projective Moduli for Polarized Manifolds, Ergebnisse der Mathematik und ihrer Grenzgebiete (3) vol. 30 (Springer-Verlag, Berlin, 1995).

[79] C. T. Woodward, 'On D. Peterson's comparison formula for Gromov-Witten invariants of G/P', Proc. Amer. Math. Soc. 133(6) (2005), 1601-1609.

[80] H. Yanagihara, 'Some results on weakly normal ring extension', J. Math. Soc. Japan 35(4) (1983), 649-661.

[81] X. Zhu, 'An introduction to affine Grassmannians and the geometric Satake equivalence', in Geometry of Moduli Spaces and Representation Theory (American Mathematical Societ, Providence, RI, 2017), 59-154. 